\title{
ENERGY INJECTION IN GAMMA-RAY BURST AFTERGLOWS
}

\author{
Tanmoy Laskar $^{1}$, Edo Berger ${ }^{1}$, Raffaella Margutti ${ }^{1}$, Daniel Perley $^{2}$, B. Ashley Zauderer ${ }^{1}$, \\ RE'EM SARI ${ }^{3}$, AND WEN-FAI Fong ${ }^{4,5}$ \\ ${ }^{1}$ Harvard-Smithsonian Center for Astrophysics, 60 Garden Street, Cambridge, MA 02138, USA \\ ${ }^{2}$ Department of Astronomy, California Institute of Technology, MC 249-17, 1200 East California Blvd, Pasadena CA 91125, USA \\ ${ }^{3}$ Racah Institute of Physics, Edmund J. Safra Campus, Hebrew University of Jerusalem, Jerusalem 91904, Israel \\ ${ }^{4}$ University of Arizona, 933 N. Cherry Ave, Tucson, AZ 85721, USA \\ Received 2015 April 14; accepted 2015 June 6; published 2015 November 10
}

\begin{abstract}
We present multi-wavelength observations and modeling of gamma-ray bursts (GRBs) that exhibit a simultaneous re-brightening in their X-ray and optical light curves, and are also detected at radio wavelengths. We show that the re-brightening episodes can be modeled by injection of energy into the blastwave and that in all cases the energy injection rate falls within the theoretical bounds expected for a distribution of energy with ejecta Lorentz factor. Our measured values of the circumburst density, jet opening angle, and beaming-corrected kinetic energy are consistent with the distribution of these parameters for long-duration GRBs at both $z \sim 1$ and $z \gtrsim 6$, suggesting that the jet launching mechanism and environment of these events are similar to that of GRBs that do not have bumps in their light curves. However, events exhibiting re-brightening episodes have lower radiative efficiencies than average, suggesting that a majority of the kinetic energy of the outflow is carried by slow-moving ejecta, which is further supported by steep measured distributions of the ejecta energy as a function of Lorentz factor. We do not find evidence for reverse shocks over the energy injection period, implying that the onset of energy injection is a gentle process. We further show that GRBs exhibiting simultaneous X-ray and optical re-brightenings are likely the tail of a distribution of events with varying rates of energy injection, forming the most extreme events in their class. Future X-ray observations of GRB afterglows with Swift and its successors will thus likely discover several more such events, while radio follow-up and multi-wavelength modeling of similar events will unveil the role of energy injection in GRB afterglows.
\end{abstract}

Key words: gamma-ray burst: general - gamma-ray burst: individual (GRB 100418A, GRB 100901A, GRB 120326A, GRB 120404A)

Supporting material: extended figures, machine-readable tables

\section{INTRODUCTION}

Gamma-ray bursts (GRBs) have traditionally been modeled as point explosions that inject $\sim 10^{51}$ erg of energy into a collimated, relativistically expanding fireball over a period of a few seconds. In this model, the subsequent afterglow radiation is synchrotron emission produced by the interaction of the relativistic ejecta with the circumburst medium. Depending on the density profile of the ambient medium, usually assumed to be either uniform ("ISM-like") or falling with radius as $r^{-2}$ ("wind-like"), this model has several verifiable predictions: smooth light curves at all frequencies from the X-rays to the radio, which rise and fall as the peak of the spectral energy distribution (SED) evolves through the observer band; a "jet break" as the expanding ejecta decelerate and begin to spread sideways; and an eventual transition to the sub-relativistic regime where the ejecta become quasi-spherical. In this framework, the energetics of the explosion and the properties of the environment can be determined from fitting light curves with the synchrotron model, while a measurement of the jet break allows for a determination of the angle of collimation of the outflow and the calculation of geometric corrections to the inferred energy.

Despite its simplicity, this model was quite successful in the study of a large number of GRB afterglows (e.g., Berger et al. 2000; Panaitescu \& Kumar 2001, 2002; Yost et al. 2003) until the launch of Swift in 2004 (Gehrels et al. 2004). With its rapid-

\footnotetext{
${ }^{5}$ Einstein Fellow.
}

response X-ray and UV/optical afterglow measurements, Swift has revolutionized the study of GRB afterglows. Rapidly available localizations have allowed detailed ground-based follow-up. X-ray observations from Swift have been even more revolutionary, revealing light curves with multiple breaks falling into a "canonical" series, consisting of a steep decay, plateau, and normal decay, sometimes with evidence for jetbreaks. While the steep decay has been associated with the prompt emission (Tagliaferri et al. 2005; O'Brien et al. 2006) and the normal and post jet-break decay phases are associated with the afterglow, the plateaus cannot be explained by the standard model (Zhang et al. 2006; however, see recent numerical calculations by Duffell \& MacFadyen 2014, which suggest that the plateaus may be a natural consequence of a coasting phase in the jet dynamics between $10^{13}$ and $10^{16} \mathrm{~cm}$ from the progenitor). In addition, short $(\Delta t / t \ll 1)$ flares that rise and decline rapidly and exhibit large flux variations $(\Delta F / F \gg 1$; Chincarini et al. 2010; Margutti et al. 2010b) are often seen superposed on the light curves. These flares are believed to be more closely associated with the GRB prompt emission than with the afterglow, and have been interpreted as late-time activity by the central engine (Falcone et al. 2006; Liang et al. 2006; Pagani et al. 2006; Romano et al. 2006; Wang et al. 2006; Lazzati \& Perna 2007; Margutti et al. 2010b; Guidorzi et al. 2015; Yu et al. 2015). Some GRBs also exhibit optical flares that do not always correspond to flares in the X-ray light curves ( $\mathrm{Li}$ et al. 2012). 
These new features of Swift X-ray light curves cannot be simply explained in the traditional picture. New physical mechanisms such as energy injection, circumburst density enhancements, structured jets, viewing angle effects, varying microphysical parameters, and gravitational micro-lensing have been invoked to explain various features of Swift X-ray light curves (Eichler \& Granot 2006; Granot et al. 2006; Nousek et al. 2006; Panaitescu et al. 2006; Toma et al. 2006; Zhang et al. 2006; Jin et al. 2007; Shao \& Dai 2007; Kong et al. 2010; Duffell \& MacFadyen 2014; Uhm \& Zhang 2014). However, although a wealth of information is available from X-ray light curves in general, definitive statements on the physical origin of these features requires synergy with observations at other wavelengths. Ultraviolet (UV), optical, near infrared (NIR), millimeter, and radio data probe distinct parts of the afterglow SED, and the various physical mechanisms are expected to influence light curves in these bands differently. Thus, a detailed analysis of GRB afterglows requires multi-wavelength data and modeling.

Of the GRBs with plateaus in their X-ray light curves, there is a small class of peculiar events that additionally exhibit an $\mathrm{X}$-ray re-brightening of a non-flaring origin $(\Delta T / T \sim 1)$, and an even smaller class where the re-brightening appears to occur simultaneously in both the optical and X-rays (Mangano et al. 2007; Li et al. 2012; Panaitescu et al. 2013). An exemplar of this latter class is GRB 120326A, which exhibits a peak in its well-sampled X-ray light curve at around $0.3 \mathrm{~d}$ together with a simultaneous optical re-brightening. Urata et al. (2014) reported optical and millimeter observations of the afterglow of GRB 120326A, and invoked synchrotron self inverse-Compton radiation from a reverse shock (RS) to explain the millimeter, optical, and X-ray light curves. By fitting energy-injection models in a wind-like circumburst environment to the X-ray and optical $R$-band light curves, Hou et al. (2014) proposed that a newborn millisecond pulsar with a strong wind was responsible for the re-brightening. Melandri et al. (2014) present multi-band optical and NIR light curves of this event, and explore various physical scenarios for the re-brightening, including the onset of the afterglow, passage of a synchrotron break frequency through the observing band, and geometrical effects.

Here we report detailed radio observations of this event spanning 4-220 GHz and 0.3 to $120 \mathrm{~d}$, making this the first GRB with an achromatic re-brightening and with such a rich multi-band data set. We perform the first broad-band modeling for this event using a physical GRB afterglow model (Granot \& Sari 2002) using methods described in Laskar et al. (2013, 2014), employing Markov Chain Monte Carlo procedures to characterize the blastwave shock. Our radio observations allow us to constrain the synchrotron self-absorption frequency, and to unambiguously locate the synchrotron peak frequency, together placing strong constraints on the nature of the X-ray/ $\mathrm{UV} /$ optical re-brightening. We find the clear signature of a jet break at all wavelengths from the radio through the X-rays, allowing us to constrain the true energetics of this event.

We next consider and test various physical processes that may cause a re-brightening in the afterglow light curve, and argue that energy injection is the most plausible mechanism. We model the re-brightening as a power-law increase in blastwave energy, self-consistently accounting for the change in the synchrotron spectrum over the injection period, and compute the fractional increase in energy during the re- brightening. We interpret the energy injection process in the context of a distribution of ejecta Lorentz factors, and provide a measurement of the power-law index of the Lorentz factor distribution.

To place our results in context, we search the Swift X-ray light curve archive for all events exhibiting a similar rebrightening, and present full multi-wavelength analyses, complete with deduced correlations between the physical parameters, for all events with radio detections that exhibit simultaneous optical and X-ray re-brightenings. This selection yields three additional events: GRBs 100418A, 100901A, and 120404A. We collect, analyze, and report all X-ray and UV data from the X-ray telescope (XRT) and UV-optical telescope (UVOT) on board Swift for these three events in addition to GRB 120326A. We also analyze and report previously unpublished archival radio observations from the Very Large Array (VLA), Submillimeter Array (SMA), and Westerbork Synthesis Radio Telescope (WSRT) for events in our sample, and the first complete multi-wavelength model fits for GRBs 100418A and 100901A. Finally, we compare our results to modeling efforts of a sample of GRBs ranging from $z \sim 1$ to $z \gtrsim 6$, as well as with a complete sample of plateaus in Swift/ XRT light curves, and thereby assess the ubiquity of the energy injection phenomenon. We infer the fractional increase in blastwave energy over the plateau phase using simple assumptions on the afterglow properties, and determine the unique characteristics of these events that result in multi-band re-brightenings. We conclude with a discussion of the results from this X-ray-only analysis and our full broad-band modeling in the context of energy injection in GRBs.

Throughout the paper, we use the following values for cosmological parameters: $\Omega_{\mathrm{m}}=0.27, \quad \Omega_{\Lambda}=0.73$, and $H_{0}=71 \mathrm{~km} \mathrm{~s}^{-1} \mathrm{Mpc}^{-1}$. All times are in the observer frame, uncertainties are at the $68 \%$ confidence level $(1 \sigma)$, and magnitudes are in the $\mathrm{AB}$ system and are not corrected for galactic extinction, unless stated otherwise.

\section{GRB PROPERTIES AND OBSERVATIONS}

GRB 120326A was discovered by the Swift Burst Alert Telescope (BAT, Barthelmy et al. 2005) on 2012 March 26 at 01:20:29 UT (Siegel et al. 2012). The burst duration is $T_{90}=26.7 \pm 0.4 \mathrm{~s}$, with a fluence of $F_{\gamma}=(1.1 \pm 0.1) \times 10^{-6} \mathrm{erg} \mathrm{cm}^{-2} \quad(15-150 \mathrm{keV} \quad$ Barthelmy et al. 2012). A bright X-ray and UV/optical afterglow was detected by Swift (Kuin et al. 2012; Siegel et al. 2012) and numerous ground-based observatories. Spectroscopic observations at the $10.4 \mathrm{~m}$ Gran Telescope Canarias provided a redshift of $z=1.798$ (Tello et al. 2012).

The burst also triggered the Fermi Gamma-ray Burst Monitor (GBM) at 01:20:31.51 UT (Collazzi 2012). The burst duration as observed by GBM is $T_{90}=11.8 \pm 1.8 \mathrm{~s}(50-300 \mathrm{keV})$ with a fluence of $(3.26 \pm 0.05) \times 10^{-6} \mathrm{erg} \mathrm{cm}^{-2}(10-1000 \mathrm{keV})$. The time-averaged $\gamma$-ray spectrum is well fit by a Band function $^{6}$, with break energy, $E_{\text {peak }}=43.9 \pm 3.9 \mathrm{keV}$, low energy index, $\alpha=-0.67 \pm 0.19$, and high-energy index, $\beta=-2.33 \pm 0.09$ Using the source redshift of $z=1.798$, the inferred isotropic equivalent $\gamma$-ray energy in the $1-10^{4} \mathrm{keV}$ rest frame energy band is $E_{\gamma, \text { iso }}=(3.15 \pm 0.12) \times 10^{52} \mathrm{erg}$.

\footnotetext{
6 From the Fermi GRB catalog for trigger 120326056 at http://heasarc.gsfc. nasa.gov/W3Browse/fermi/fermigbrst.html.
} 


\subsection{X-ray: Swift/XRT}

The Swift XRT, (Burrows et al. 2005b) began observing the field at $69 \mathrm{~s}$ after the BAT trigger, leading to the detection of an X-ray afterglow. The source was localized to R.A. $=18^{\mathrm{h}} 15^{\mathrm{m}} 37^{\mathrm{s}} .06$, decl. $=+69^{\mathrm{d}} 15^{\prime} 35^{\prime \prime} .4(\mathrm{~J} 2000)$, with an uncertainty radius of $1.4 \operatorname{arcsec}\left(90 \%\right.$ containment). ${ }^{7}$ XRT continued observing the afterglow for $18.7 \mathrm{~d}$ in photon counting mode, with the last detection at $5.2 \mathrm{~d}$.

We extracted XRT PC-mode spectra using the on-line tool on the Swift website (Evans et al. 2007, 2009). ${ }^{8}$ We analyzed the data after the end of the steep decay at $400 \mathrm{~s}$ using the latest version of the HEASOFT package (v6.14) and corresponding calibration files. We used Xspec (v12.8.1) to fit all available PC-mode data, assuming a photoelectrically absorbed powerlaw model (tbabs $\times$ ztbabs $\times$ pow) and a Galactic neutral hydrogen column density of $N_{\mathrm{H}, \mathrm{MW}}=6.3 \times 10^{20} \mathrm{~cm}^{-2}$ (for consistency with the value used on the Swift website), fixing the source redshift at $z=1.798$. Our best-fit model has a photon index of $\Gamma=1.85 \pm 0.04$ and excess absorption corresponding to a neutral hydrogen column (assuming solar metallicity) of $N_{\mathrm{H}, \text { int }}=(4.1 \pm 0.7) \times 10^{21} \mathrm{~cm}^{-2}$ intrinsic to the host galaxy (C-stat $=497.1$ for 570 degrees of freedom). We divided the PC-mode data into six roughly equal time bins and extracted time-resolved PC-mode spectra to test for spectral evolution. We do not find clear evidence for significant spectral evolution over this period. In the following analysis, we take the $0.3-10 \mathrm{keV}$ count rate light curve from the above website together with $\Gamma_{\mathrm{X}}=1.85$ (corresponding to $\beta_{\mathrm{X}} \equiv 1-\Gamma_{\mathrm{X}}=-0.85$ ) for the $\mathrm{PC}$ mode to compute the $1 \mathrm{keV}$ flux density. We combine the uncertainty in flux calibration based on our spectral analysis (2.7\%) in quadrature with the statistical uncertainty from the on-line light curve. For the WTmode, we convert the count rate light curve to a flux-calibrated light curve using $\Gamma=3.57$ and a count-to-flux conversion factor of $7 \times 10^{-11} \mathrm{erg} \mathrm{cm}^{-2} \mathrm{ct}^{-1}$ as reported on the on-line spectral analysis.

The WT-mode X-ray light curve declines rapidly as $t^{-3.3 \pm 0.1}$. Swift switched to collecting data in PC-mode at $150 \mathrm{~s}$. The PCmode data between 150 and $300 \mathrm{~s}$ continue to decline rapidly as $t^{-3.8 \pm 0.4}$, followed by a plateau where the count rate evolves as $t^{-0.22 \pm 0.05}$. This part of the lightcurve before $300 \mathrm{~s}$ is likely dominated by the high-latitude prompt emission (Willingale et al. 2010), and we therefore do not consider the X-ray observations before $300 \mathrm{~s}$ in our afterglow modeling. About $0.16 \mathrm{~d}$ after the trigger, the $\mathrm{X}$-ray count rate begins rising and peaks at around $0.41 \mathrm{~d}$. This re-brightening is unusual for X-ray afterglows and we discuss this feature further in Section 3.1. The XRT count rate light curve after $1.4 \mathrm{~d}$ can be fit by a single power law with a decline rate of $\alpha_{\mathrm{X}}=-2.29 \pm 0.16$.

\subsection{UV/Optical: Swift/UVOT}

The Swift UV/Optical Telescope (UVOT; Roming et al. 2005) observed GRB 120326A using 6 filters spanning the central wavelength range $\lambda_{\mathrm{c}}=1928 \AA(W 2)$ to $\lambda_{\mathrm{c}}=5468 \AA(v)$ beginning $67 \mathrm{~s}$ after the burst. The afterglow was detected in the initial exposures at R.A. $=18^{\mathrm{h}} 15^{\mathrm{m}} 37^{\mathrm{s}} \cdot 13$, decl. $=+69^{\mathrm{d}}$ $15^{\prime} 35^{\prime \prime} .36$ (J2000) (90\% confidence, Kuin et al. 2012), and exhibited a clear re-brightening concomitant with the peak in the

\footnotetext{
http://www.swift.ac.uk/xrt_positions/00518626/

8 http://www.swift.ac.uk/xrt_spectra/00518626/
}

Table 1

Swift/UVOT Observations of GRB 120326A

\begin{tabular}{lccccc}
\hline \hline $\begin{array}{l}t-t_{0} \\
(\text { days })\end{array}$ & Filter & $\begin{array}{c}\text { Frequency } \\
(\mathrm{Hz})\end{array}$ & $\begin{array}{c}\text { Flux } \\
\text { Density } \\
(\mu \mathrm{Jy})\end{array}$ & $\begin{array}{c}\text { Uncertainty } \\
(\mu \mathrm{Jy})\end{array}$ & $\begin{array}{c}\text { Detection? } \\
(1=\mathrm{Yes})\end{array}$ \\
\hline 0.00165 & White & $8.64 \mathrm{e}+14$ & 8.89 & 2.71 & 1 \\
0.00354 & $u$ & $8.56 \mathrm{e}+14$ & 20.7 & 11.5 & 0 \\
0.0523 & $b$ & $6.92 \mathrm{e}+14$ & 39 & 6.23 & 1 \\
0.0546 & White & $8.64 \mathrm{e}+14$ & 15.6 & 2.55 & 1 \\
0.0558 & \multirow{2}{*}{ wvwl } & $1.16 \mathrm{e}+15$ & 10.9 & 3.29 & 1 \\
\hline
\end{tabular}

Note.

${ }^{\mathrm{a}}$ In cases of non-detections, we report the formal flux density measurement from aperture photometry.

(This table is available in its entirety in machine-readable form.)

X-ray light curve. We analyzed the UVOT data using the latest version of HEASOFT (v. 6.14) and corresponding calibration files. We performed photometry with a $5^{\prime \prime}$ aperture and used a $90^{\prime \prime}$ annulus with foreground sources masked to estimate the background. We list our derived fluxes in Table 1.

\subsection{Optical/NIR: Palomar Observations and GCN Circulars}

We observed GRB 120326A with the Wide-field Infrared Camera (Wilson et al. 2003) on the Palomar 200-inch telescope beginning on 2012 March 30.48 UT. We acquired a series of nine $75 \mathrm{~s} J$-band exposures, followed by a series of nine $75 \mathrm{~s}$ $K_{s}$-band exposures. We reduced the data following standard IR imaging techniques using a modified version of the WIRCSOFT pipeline and performed aperture photometry of the afterglow in the combined, stacked exposures relative to 2MASS standards in the field.

We also carried out a series of imaging observations of GRB 120326A with the robotic Palomar 60-inch telescope (Cenko et al. 2006) on the nights of 2012 March 31, April 02, April $03\left(r^{\prime}\right.$ and $\left.i^{\prime}\right)$, April 04, April 08, and April 16 ( $r^{\prime}$ only). We reduced the images using the P60 automated pipeline and performed aperture photometry relative to secondary standard stars calibrated via separate observations of Landolt (2009) standards.

Finally, we collected other optical and NIR observations of GRB 120326A reported through the Gamma-ray Burst Coordinates Network (GCN) Circulars and converted all photometry to flux densities. We also include the optical photometry of the afterglow published by Melandri et al. (2014) and Urata et al. (2014) in our analysis. We list our complete compilation of optical observations of this burst, together with our P60 and P200 observations, in Table 2.

\subsection{Sub-millimeter: CARMA and SMA}

We observed GRB 120326A with the Combined Array for Research in Millimeter Astronomy (CARMA) beginning on 2012 March 30.56 UT (4.52 d after the burst) in continuum wideband mode with $\approx 8 \mathrm{GHz}$ bandwidth (16 windows, $487.5 \mathrm{MHz}$ each) at a mean frequency of $93 \mathrm{GHz}$. Following an initial detection (Perley et al. 2012), we obtained two additional epochs. Weather conditions were excellent for each epoch, with the first observation being taken in $\mathrm{C}$ configuration (maximum baseline $\sim 370 \mathrm{~m}$ ), and the following observations in D configuration (maximum baseline $\sim 145 \mathrm{~m}$ ). For all observations, we utilized 3C371 for bandpass, amplitude and 
Table 2

Optical Observations of GRB 120326A

\begin{tabular}{|c|c|c|c|c|c|c|c|c|}
\hline $\begin{array}{l}t-t_{0} \\
\text { (days) }\end{array}$ & Observatory & $\begin{array}{l}\text { Telescope/ } \\
\text { Instrument }\end{array}$ & Filter & $\begin{array}{c}\text { Frequency } \\
(\mathrm{Hz})\end{array}$ & $\begin{array}{l}\text { Flux Density } \\
\qquad(\mu \mathrm{Jy})\end{array}$ & $\begin{array}{c}\text { Uncertainty } \\
(\mu \mathrm{Jy})\end{array}$ & $\begin{array}{l}\text { Detection? } \\
(1=\text { Yes })\end{array}$ & Reference \\
\hline 0.000291 & TNO & ROTSE & $C R$ & $4.56 \mathrm{e}+14$ & $4.04 \mathrm{e}+03$ & $1.35 \mathrm{e}+03$ & 0 & Rujopakarn \& Flewelling (2012) \\
\hline 0.001 & TNO & ROTSE & $C R$ & $4.56 \mathrm{e}+14$ & 926 & 308 & 0 & Rujopakarn \& Flewelling (2012) \\
\hline 0.00189 & Calern & TAROT & $R$ & $4.56 \mathrm{e}+14$ & 161 & 51.2 & 1 & Klotz et al. (2012) \\
\hline 0.00389 & Liverpool & RINGO2 & $R$ & $4.56 \mathrm{e}+14$ & 120 & 21.6 & 1 & Melandri et al. (2014) \\
\hline 0.00545 & Crni_Vrh & Cicocki & $R$ & $4.56 \mathrm{e}+14$ & 95.1 & 13.1 & 1 & Melandri et al. (2014) \\
\hline
\end{tabular}

Notes.

${ }^{a}$ In cases of non-detections, we report the $3 \sigma$ upper limit on the flux density.

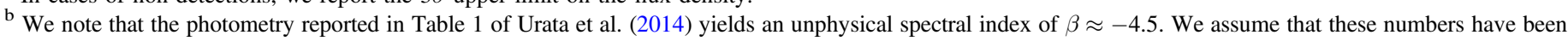

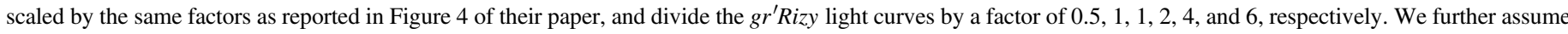
that the data have not been corrected for galactic extinction.

(This table is available in its entirety in machine-readable form.)

Table 3

Millimeter and Radio Observations of GRB 120326A

\begin{tabular}{|c|c|c|c|c|c|c|c|}
\hline $\begin{array}{l}\text { Date } \\
\text { (UT) }\end{array}$ & $\begin{array}{l}t-t_{0} \\
\text { (days) }\end{array}$ & Observatory $^{\mathrm{a}}$ & $\begin{array}{l}\text { Frequency } \\
\quad(\mathrm{GHz})\end{array}$ & $\begin{array}{l}\text { Integration Time } \\
\text { (minutes) }\end{array}$ & $\begin{array}{l}\text { Flux Density } \\
\text { (mJy) }\end{array}$ & $\begin{array}{c}\text { Uncertainty }^{\mathrm{b}} \\
(\mathrm{mJy})\end{array}$ & $\begin{array}{l}\text { Detection? } \\
(1=\text { Yes })\end{array}$ \\
\hline 2012 Mar 30.56 & 4.55 & CARMA & 92.5 & 60.3 & 3.38 & 0.87 & 1 \\
\hline 2012 Apr 5.53 & 10.51 & CARMA & 92.5 & 73.0 & 1.46 & 0.42 & 1 \\
\hline 2012 Apr 10.40 & 15.51 & CARMA & 92.5 & 291.9 & 0.476 & 0.17 & 1 \\
\hline 2012 Mar 26.43 & 0.504 & $\mathrm{SMA}^{\mathrm{c}}$ & 222 & 386 & 3.3 & 0.90 & 1 \\
\hline 2012 Mar 27.53 & 1.55 & $\mathrm{SMA}^{\mathrm{c}}$ & 222 & 227 & 2.4 & 0.80 & 1 \\
\hline
\end{tabular}

Notes.

"The letter following "VLA" indicates the array configuration.

b $1 \sigma$ statistical uncertainties from AIPS task JMFIT (CARMA and SMA observations) or CASA task IMFIT (VLA observations).

c PI: Y. Urata.

(This table is available in its entirety in machine-readable form.)

phase gain calibration, and MWC349 for flux calibration. We reduced the data using standard procedures in MIRIAD (Sault et al. 1995). In the last epoch on April 10, we also observed 3C273 and 3C279 and utilized these observations as independent checks on the bandpass and flux calibration. We summarize our observations in Table 3.

The SMA; (Ho et al. 2004) observed GRB 120326A at a mean frequency of $222 \mathrm{GHz}(1.3 \mathrm{~mm}$; lower sideband centered at $216 \mathrm{GHz}$, upper sideband at $228 \mathrm{GHz}$; PI: Urata). Six epochs of observations were obtained between 2012 March 26.43 UT ( $0.37 \mathrm{~d}$ after the burst) and 2012 April 11.64 UT (16.6 d after the burst). These observations have been reported in Urata et al. (2014). We carried out an independent reduction of the data using standard MIR IDL procedures for the SMA, followed by flagging, imaging and analysis in MIRIAD and the Astronomical Image Processing System; (Greisen 2003, p. 109). We utilized 3C279 for bandpass calibration in all but the last epoch, where we utilized J1924-292. We utilized J1800+784 and $\mathrm{J} 1829+487$ for gain calibration. ${ }^{9}$ We utilized MWC349a for flux calibration, determining a flux density of $1.42 \mathrm{Jy}$ for J1800 +784 , consistent with flux values in the SMA catalog at similar times. We utilized this flux throughout all observations

\footnotetext{
9 The gain calibrators were separated by more than $20^{\circ}$ from the source; thus some decoherence, resulting in a systematic reduction of the observed flux is possible.
}

(not every epoch contained useful flux calibrator scans) and scaled the gains appropriately. We note an uncertainty in the absolute flux density scale of $\approx 15 \%$. We detect a source at R.A. $=18^{\mathrm{h}} 15^{\mathrm{m}} 37^{\mathrm{s}} .15( \pm 0.02)$ s, decl. $=+69^{\mathrm{d}} 15^{\prime}$ $35^{\prime \prime} .41$ ( $\pm 0.14 ;$ J2000). Two epochs obtained on March 31.40 and April 6.51 showed poor noise characteristics, and we do not include them in our analysis. We measure poorer noise statistics in the observations than reported by Urata et al. (2014), and also do not detect the source in the epoch at $3.53 \mathrm{~d}$, contrary to the previous analysis of this data set. We report the results of our analysis in Table 3.

\subsection{Radio: VLA}

We observed the afterglow at $\mathrm{C}(4-7 \mathrm{GHz}), \mathrm{K}(18-25 \mathrm{GHz})$, and $\mathrm{Ka}(30-38 \mathrm{GHz})$ bands using the Karl G. Jansky VLA starting $5.45 \mathrm{~d}$ after the burst. We detected and tracked the flux density of the afterglow over eight epochs until $122 \mathrm{~d}$ after the burst, until the source had either faded below or was barely detectable at the $3 \sigma$-level at all frequencies. Depending on the start time of the observations, we used either $3 \mathrm{C} 286$ or $3 \mathrm{C} 48$ as the flux and bandpass calibrator; we used J1806+6949 or $\mathrm{J} 1842+6809$ as gain calibrator depending on the array configuration and observing frequency. We carried out data reduction using the Common Astronomy Software Applications. We list our VLA photometry in Table 3. 
Table 4

Parameters for Broken Power-law Fit to X-ray/UV/Optical Re-brightening for GRB 120326A

\begin{tabular}{|c|c|c|c|c|}
\hline & XRT & $U$-band & $z$-band & Joint $^{\mathrm{a}}$ (MCMC) \\
\hline Break time, $t_{\mathrm{b}}(\mathrm{d})$ & $0.41 \pm 0.02$ & $0.31 \pm 0.04$ & $0.31^{\mathrm{b}}$ & $0.40 \pm 0.02$ \\
\hline Flux density at $t_{\mathrm{b}}, F_{\mathrm{b}}(\mu \mathrm{Jy})$ & $1.23 \pm 0.05$ & $66 \pm 7$ & $255 \pm 10$ & $\ldots$ \\
\hline Rise rate, $\alpha_{1}$ & $0.85 \pm 0.19$ & $0.89 \pm 0.21$ & $0.59 \pm 0.03$ & $0.52 \pm 0.06$ \\
\hline Decay rate, $\alpha_{2}$ & $-1.22 \pm 0.18$ & $-1.10 \pm 0.29$ & $-0.94 \pm 0.05$ & $-1.10 \pm 0.10$ \\
\hline Smoothness, $y$ & $5.0 \pm 4.0$ & $5.0^{\mathrm{b}}$ & $5.0^{\mathrm{b}}$ & $17 \pm 10$ \\
\hline
\end{tabular}

Notes.

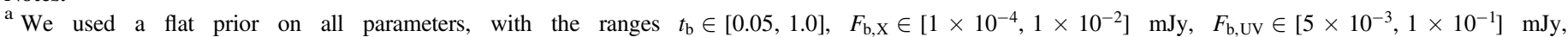
$F_{\mathrm{b}, \mathrm{z}} \in\left[1 \times 10^{-2}, 2\right] \mathrm{mJy}, \alpha_{1} \in[0.1,5.0], \alpha_{2} \in[-5.0,0.0]$, and $y \in[0.5,30]$.

$\mathrm{b}$ Parameters fixed during fit (see the text for details).

\section{BASIC CONSIDERATIONS}

We consider the X-ray to radio afterglow of GRB $120326 \mathrm{~A}$ in the context of the standard synchrotron afterglow model, where the afterglow emission is produced by synchrotron radiation from a non-thermal distribution of electrons. The electron energy distribution is assumed to be a power law with electron number density, $n(\gamma) \propto \gamma^{-p}$, for $\gamma>\gamma_{\min }$; here $\gamma_{\min }$ is the minimum Lorentz factor and the electron energy index, $p$ is expected to lie between 2 and 3 . This electron distribution results in a synchrotron spectrum that can be described by a series of power-law segments connected at "break frequencies": the self-absorption frequency, $\nu_{\mathrm{a}}$, below which synchrotron self-absorption suppresses the flux, the characteristic synchrotron frequency, $\nu_{\mathrm{m}}$, which corresponds to emission from electrons with $\gamma=\gamma_{\min }$, and the cooling frequency, $\nu_{\mathrm{c}}$, above which synchrotron cooling is important; the flux density at $\nu_{\mathrm{m}}$ sets the overall flux normalization. The synchrotron model is described in detail in Sari et al. (1998), Chevalier \& Li (2000), and Granot \& Sari (2002).

\subsection{X-Ray/UV/Optical Re-brightening at $0.4 d$}

A prominent feature of the afterglow light curve of GRB $120326 \mathrm{~A}$ is a re-brightening at about $0.4 \mathrm{~d}$. We quantify the shape of the light curve at the re-brightening by fitting the $\mathrm{X}$-ray data with a smoothly joined broken power law of the form

$$
F_{\nu}=F_{\mathrm{b}}\left(\frac{\left(t / t_{\mathrm{b}}\right)^{-y \alpha_{1}}+\left(t / t_{\mathrm{b}}\right)^{-y \alpha_{2}}}{2}\right)^{-1 / y}
$$

where $t_{\mathrm{b}}$ is the break time, $F_{\mathrm{b}}$ is the flux at the break time, $\alpha_{1}$ and $\alpha_{2}$ are the temporal indices before and after the break, respectively, and $y$ is the sharpness of the break. ${ }^{10}$ The $\mathrm{X}$-ray data before $0.15 \mathrm{~d}$ exhibit a plateau and we therefore restrict the fit to span 0.15 to $1.25 \mathrm{~d}$. We use the Python function curve_fit to estimate these model parameters and the associated covariance matrix. Our best-fit parameters are $t_{\mathrm{b}}=(0.41 \pm 0.02) \mathrm{d}, \alpha_{1}=0.85 \pm 0.19, \alpha_{2}=-1.22 \pm 0.18$, and $y=5 \pm 4$ (Figure 1 and Table 4).

The optical data are more sparsely sampled than the X-ray observations. We fit the $U$-band data between $0.05 \mathrm{~d}$ and $1.25 \mathrm{~d}$ after fixing $y=5$ as suggested by the fit to the better-sampled

\footnotetext{
${ }^{10} \mathrm{We}$ impose a floor of $12 \%$ on the uncertainty of each data point, as explained in Section 4.
}

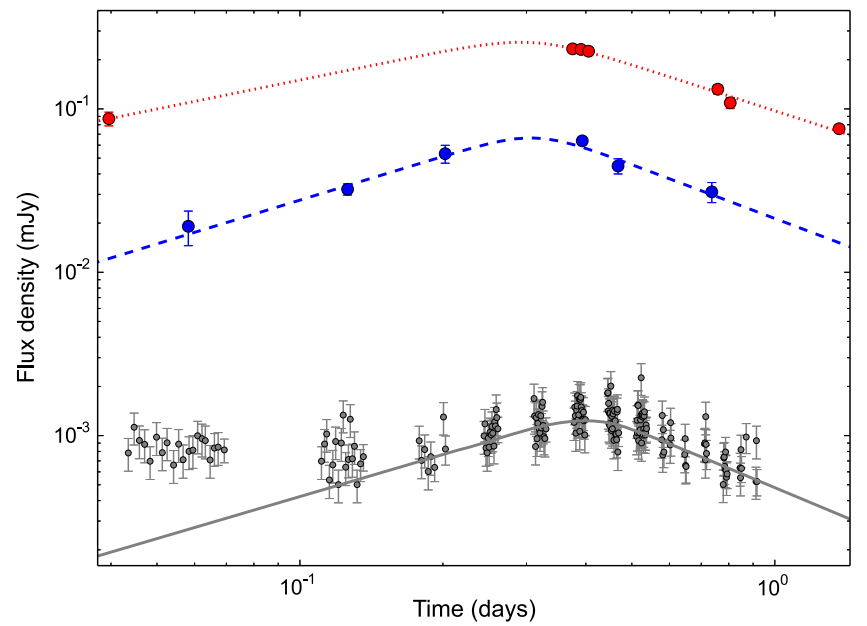

Figure 1. Broken power-law fits to the X-ray (gray, solid), $U$-band (blue, dashed), and $z^{\prime}$-band (red, dotted) light curves for GRB 120326A near the rebrightening around $0.35 \mathrm{~d}$. X-ray points before $0.15 \mathrm{~d}$ are not included in the fit. Errorbars at $z$-band are typically smaller than the size of the plotted symbols. The lines correspond to the independent fits at the three frequencies. The best-fit parameters are listed in Table 4 .

X-ray light curve. Our derived value of the peak time, $t_{\mathrm{b}}=0.31 \pm 0.04$ is marginally earlier than, but close to the peak time of the X-ray light curve. The rise and decay rate in $U$-band are also statistically consistent with those derived from the X-ray light curve. Similarly, we fit the $z$-band light curve between $0.04 \mathrm{~d}$ and $1.4 \mathrm{~d}$, fixing $y=5$. Since this time range includes a single point prior to the peak, the peak time and rise rate are degenerate in the fit. We therefore fix $t_{\mathrm{b}}=0.31$ as derived from the $U$-band fit. The best-fit parameters are listed in Table 4, and are consistent with those derived for the UV and $\mathrm{X}$-ray light curves.

Finally, we fit the X-ray, UV, and interpolated optical/NIR data jointly, where the three light curves are constrained to the same rise and decay rate, time of peak, and sharpness of the break, with independent normalizations in the three bands. Using a Markov Chain Monte Carlo simulation using EMCEE (Foreman-Mackey et al. 2013), we find $t_{\mathrm{b}}=0.40$ $\pm 0.01, \alpha_{1}=0.52 \pm 0.06$, and $\alpha_{2}=-1.10 \pm 0.10$.

In summary, the X-ray/UV/optical light curves exhibit a prominent peak, nearly simultaneously in multiple bands (X-rays through the optical), which, therefore, cannot be related to the passage of a synchrotron break frequency. We explore various explanations for this behavior in Section 5.2.4. 


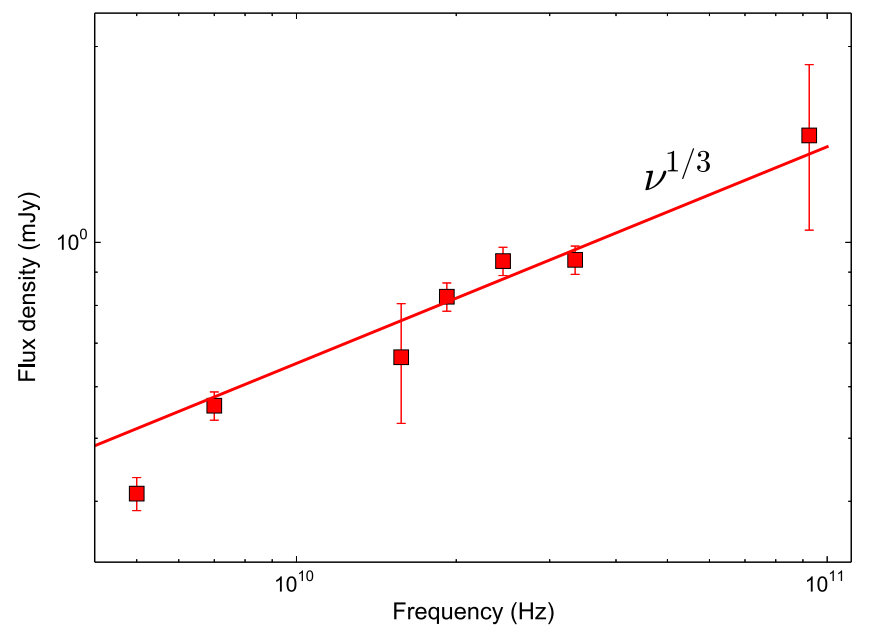

Figure 2. Afterglow SED for GRB $120326 \mathrm{~A}$ at $9 \mathrm{~d}$ from 5 to $92.5 \mathrm{GHz}$ together with a best-fit power-law fit to the data above $7 \mathrm{GHz}$. The spectrum is optically thin with a spectral index of $\nu^{0.35 \pm 0.03}$ from 7 to $92.5 \mathrm{GHz}$. The $5 \mathrm{GHz}$ observation shows evidence of synchrotron self-absorption.

\subsection{X-Ray/Radio Steep Decline: $\nu_{m}$ and $t_{\text {jet }}$}

The X-ray data at $\gtrsim 1.4 \mathrm{~d}$ exhibit a steep decline, with $\alpha_{\mathrm{X}}=-2.29 \pm 0.16$. In the standard afterglow model a steep decline of $\alpha \approx-p \lesssim-2$ at frequencies above $\nu_{\mathrm{m}}$ is expected after the "jet-break" $\left(t_{\text {jet }}\right)$, when the bulk Lorentz factor, $\Gamma$, decreases below the inverse opening angle of the jet, $1 / \theta_{\text {jet }}$, and the edges of the collimated outflow become visible. The $r^{\prime}$-band light curve also exhibits a shallow-to-steep transition at $\approx 1.5 \mathrm{~d}$, with a post-break decay rate of $\alpha_{\mathrm{R}}=-2.27 \pm 0.11$, consistent with the X-rays. These observations suggest that the jet break occurs at about $1.5 \mathrm{~d}$. We now consider whether this interpretation is consistent with the radio observations.

The multi-wavelength radio SED at $9 \mathrm{~d}$ is well-fit by a single power law with spectral index, $\beta=1 / 3$ from 7 to $93 \mathrm{GHz}$ (Figure 2 and Section 3.4), indicating that $\nu_{\mathrm{m}}>93 \mathrm{GHz}$ at this time. In the absence of a jet break, we would expect the flux density below $\nu_{\mathrm{m}}$ to remain constant (for a wind-like circumburst environment) or rise with time (for a constant density circumburst environment). However, the radio light curves decline after $4.6 \mathrm{~d}$ at all frequencies from $15 \mathrm{GHz}$ to $93 \mathrm{GHz}$. The combination of $\nu_{\mathrm{m}}>93 \mathrm{GHz}$ at $9 \mathrm{~d}$ and the declining light curve in the radio bands is only possible in the standard afterglow model if a jet break has occurred before $4.6 \mathrm{~d}$. This is consistent with the steepening observed in the $\mathrm{X}$-ray and optical light curves at $\approx 1.5 \mathrm{~d}$, suggesting that $t_{\text {jet }} \approx 1.5 \mathrm{~d}$.

We note that the flux at a given frequency decays steeply following a jet break only once $\nu_{\mathrm{m}}$ has crossed the observing frequency; thus the steepening in the radio light curves is expected to be delayed past that of the steepening in the X-ray and optical light curves until $\nu_{\mathrm{m}}$ passes through the radio band. We find that the $7 \mathrm{GHz}$ light curve is consistent with being flat to $50 \mathrm{~d}$, after which it declines rapidly with $\alpha_{\text {radio }} \approx-2$. This suggests that $\nu_{\mathrm{m}}$ crosses the $7 \mathrm{GHz}$ band at around $50 \mathrm{~d}$. Since $\nu_{\mathrm{m}}$ is expected to decline as $t^{-2}$ following the jet break, we have $\nu_{\mathrm{m}} \approx 8 \times 10^{12} \mathrm{~Hz}$ at $1.5 \mathrm{~d}$ if we take $t_{\text {jet }} \approx 1.5$ days. Thus $\nu_{\mathrm{m}}$ is below both the X-rays and the optical frequencies at $t=t_{\text {jet }}$, consistent with the steepening being observed around the same time in the X-ray and optical bands.
To summarize, a simultaneous steepening in the X-ray and optical light curves between 1 and $2 \mathrm{~d}$ indicates that $t_{\text {jet }} \approx 1.5$ d. Taken together with a similar steepening observed in the $7 \mathrm{GHz}$ radio light curve around $50 \mathrm{~d}$, we find $\nu_{\mathrm{m}} \approx 7 \mathrm{GHz}$ at $50 \mathrm{~d}$.

\subsection{The Circumburst Density Profile and the Location of $\nu_{c}$}

The density profile in the immediate (sub-persec scale) environment of the GRB progenitor impacts the hydrodynamic evolution of the shock powering the GRB afterglow. The evolution of the shock Lorentz factor is directly reflected in the afterglow light curves at all frequencies and multi-band modeling of GRB afterglows therefore allows us to disentangle different density profiles. Since the progenitors of long-duration GRBs are believed to be massive stars, the circumburst density structure is expected to be shaped by the stellar wind of the progenitor into a profile that falls of as $\rho(r)=A r^{-2}$ with radius $r$. Here $A=\dot{M}_{w} / 4 \pi V_{w} \equiv 5 \times 10^{11} A_{*} \mathrm{~g} \mathrm{~cm}^{-1}$ is a constant proportional to the progenitor mass-loss rate $\dot{M}_{w}$ (assumed constant), for a given wind speed, $V_{w}$ (Chevalier \& Li 2000), and $A_{*}$ is a dimensionless parametrization, corresponding to $\dot{M}_{w}=1 \times 10^{-5} M_{\odot} \mathrm{yr}^{-1}$ and $V_{w}=1000 \mathrm{~km} \mathrm{~s}^{-1}$. Alternatively, the shock may directly encounter the uniform interstellar medium (ISM). Both wind- and ISM-like environments have been inferred for different events from previous observations of GRB afterglows (e.g., Panaitescu \& Kumar 2000, 2001, 2002; Harrison et al. 2001; Yost et al. 2002; Frail et al. 2003; Chandra et al. 2008; Cenko et al. 2010, 2011; Laskar et al. 2013, 2014). Here, we explore the constraints on the progenitor environment of GRB 120326A.

The spectral index between the PAIRITEL $K$-band observation at $1.4 \mathrm{~d}$ (Morgan 2012) and the X-rays is $\beta_{\mathrm{NIR}, \mathrm{X}}=$ $-0.96 \pm 0.05$ (Figure 3 ), which is consistent with the X-ray spectral index of $\beta_{\mathrm{X}}=-0.85 \pm 0.04$ (Section 2.1) at $2 \sigma$, suggesting that the NIR, optical, and X-ray bands are located on the same power-law segment of the afterglow SED at $1.4 \mathrm{~d}$. At the same time, the spectral index within the NIR/optical bands is $\beta=-1.80 \pm 0.16$, indicating that extinction is present.

Since $\beta_{\mathrm{NIR}, \mathrm{X}} \approx \beta_{\mathrm{X}} \approx-0.90$, the cooling frequency, $\nu_{\mathrm{c}}$ must lie either below the NIR or above the X-rays at $1.4 \mathrm{~d}$. For $\nu_{\mathrm{c}}<\nu_{\mathrm{IR}}$, we would infer an electron energy index, $p \approx 1.8$ and a light curve decay rate of $\alpha \approx-0.85$ regardless of the circumburst density profile. On the other hand, $\nu_{\mathrm{c}}>\nu_{\mathrm{X}}$ requires $p \approx 2.8$ with $\alpha \approx-1.35$ for a constant density environment and $\alpha \approx-1.85$ for a wind-like environment. Actual measurements of the light curve decay rate at $\approx 1.4 \mathrm{~d}$ are complicated by the presence of the jet break at around this time. The optical and X-ray light curves decline as $\alpha_{\mathrm{R}}=$ $-2.05 \pm 0.13$ and $\alpha_{\mathrm{X}}=2.29 \pm 0.16$ after the jet break, with the expected decay rate being $\alpha \approx-p$ (Rhoads 1999; Sari et al. 1999). This indicates $p \approx 2$ (Section 3.2) and $\nu_{\mathrm{c}}<\nu_{\mathrm{m}}$ for an ISM model. Upon detailed investigation (Section 4), we find that a $p \approx 2$ model with an ISM-like environment fits the data after the re-brightening well. This model additionally requires $\nu_{\mathrm{c}}<\nu_{\mathrm{m}}$ (fast cooling) until $\approx 2 \mathrm{~d}$. For completeness, we present our investigation of the wind model in Appendix B.

\subsection{Location of $\nu_{a}$}

The radio SED from 7 to $93 \mathrm{GHz}$ at $\approx 9$ days is optically thin with a spectral slope of $\beta=0.33 \pm 0.04$ (Figure 2), suggesting 


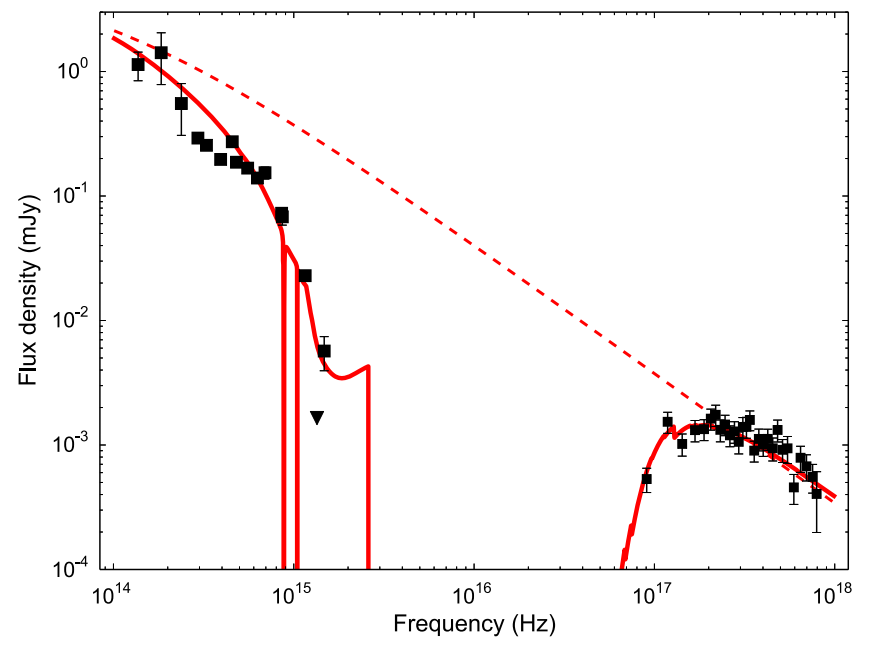

Figure 3. Afterglow SED for GRB $120326 \mathrm{~A}$ at $0.33 \mathrm{~d}$ from the NIR to the $\mathrm{X}$-rays together with the best-fit forward shock model (red, solid). The optical and UV data exhibit a clear decrement due to extinction in the host galaxy. The solid line is the SED from the highest-likelihood model and the dashed curve indicates the SED in the absence of absorption along the line of sight to the GRB by the Milky Way and the GRB host (Section 5.1). The spectral break apparent in the model SED at $\approx 10^{14} \mathrm{~Hz}$ is $\nu_{\mathrm{m}}$. The data above $\sim 9 \times 10^{14} \mathrm{~Hz}$ (Ly $\alpha$ in GRB rest frame) are likely affected by absorption by the intergalactic medium (IGM) along the line of sight.

that $\nu_{\mathrm{m}}$ lies above $93 \mathrm{GHz}$ and $\nu_{\mathrm{a}}$ lies below $7 \mathrm{GHz}$ at this time. This is consistent with the passage of $\nu_{\mathrm{m}}$ through $7 \mathrm{GHz}$ at $50 \mathrm{~d}$ inferred in Section 3.2. The spectral index between 5 and $7 \mathrm{GHz}$ at $9 \mathrm{~d}, \beta=0.92 \pm 0.23$, is steeper than $\nu^{1 / 3}$. This may suggest that the synchrotron self-absorption frequency, $\nu_{\mathrm{a}} \approx$ $5 \mathrm{GHz}$. However, this spectral index does not show a monotonic trend with time. Since this part of the radio spectrum is strongly affected by interstellar scintillation (ISS) in the ISM of the Milky Way, a unique interpretation of the observed spectral index is difficult at these frequencies. This difficulty in constraining $\nu_{\mathrm{a}}$ results in degeneracies in the physical parameters. We return to this point in Section 5.1.

\section{MULTI-WAVELENGTH MODELING}

Although the panchromatic peak at $0.4 \mathrm{~d}$ is a unique feature of GRB 120326A, the X-ray, optical, and radio light curves of this event exhibits standard afterglow features after this time, with evidence for an un-broken power law spectrum extending from the optical to the X-rays (Section 3.3), a $\nu^{1 / 3}$ spectrum in the radio (Section 3.4), and evidence for a jet break at $t_{\text {jet }} \approx 1.5 \mathrm{~d}$. (Section 3.2). We therefore determine the physical properties of this event by using observations after the X-ray/optical peak. We model the data at $\gtrsim 0.4 \mathrm{~d}$ as arising from the afterglow blastwave, using the smoothly connected power-law synchrotron spectra described by Granot \& Sari (2002). We compute the break frequencies and normalizations using the standard parameters: the fractions of the blastwave energy imparted to relativistic electrons $\left(\epsilon_{\mathrm{e}}\right)$ and magnetic fields $\left(\epsilon_{B}\right)$, the kinetic energy $\left(E_{\mathrm{K}, \text { iso }}\right)$, and the circumburst density $\left(n_{0}\right)$. We also use the SMC extinction curve ${ }^{11}$ (Pei 1992) to model the extinction in the host galaxy $\left(A_{\mathrm{V}}\right)$. Since the $R$-band light curve flattens at $\approx 10$

\footnotetext{
11 Our previous work shows negligible changes in the blastwave parameters with LMC and Milky Way-like extinction curves (Laskar et al. 2014).
}

days due to contribution from the host, we fit for the $R$-band flux density of the host galaxy as an additional free parameter.

The various possible orderings of the spectral break frequencies (e.g., $\nu_{\mathrm{m}}<\nu_{\mathrm{c}}$ : "slow cooling" and $\nu_{\mathrm{c}}<\nu_{\mathrm{m}}$ : "fast cooling") give rise to five possible shapes of the afterglow SED (Granot \& Sari 2002). Due to the hydrodynamics of the blastwave, the break frequencies evolve with time and the SED transitions between spectral shapes. To preserve smooth light curves when break frequencies cross and the spectral shape changes, we employ the weighting schemes described in Laskar et al. (2014) to compute the afterglow SED as a function of time. To efficiently and rapidly sample the available parameter space, we carry out a Markov Chain Monte Carlo (MCMC) analysis using a python implementation of the ensemble MCMC sampler EMCEE (Foreman-Mackey et al. 2013). For a detailed discussion of our modeling scheme, see Laskar et al. (2014). To account for heterogeneity of UV/ Optical/NIR data collected from different observatories, we usually impose an uncertainty floor of $5 \%$ prior to fitting with our modeling software. For this GRB, we find that the fit is driven by the optical data at the expense of fits at the radio and $\mathrm{X}$-ray bands, which we mitigate by increasing the uncertainty floor further to $12 \%$. We additionally correct for the effect of inverse Compton cooling (Appendix A).

\section{RESULTS FOR GRB 120326A}

\subsection{Multi-wavelength Model at $\gtrsim 0.4 d$}

In confirmation of the basic analysis presented in Section 3.3, our highest likelihood model (Figure 4) has $p \approx 2.09$, $\epsilon_{\mathrm{e}} \approx 0.33, \epsilon_{B} \approx 0.33, n_{0} \approx 0.27 \mathrm{~cm}^{-3}, E_{\mathrm{K} \text {,iso }} \approx 1.4 \times 10^{53} \mathrm{erg}$, $t_{\text {jet }} \approx 1.5 \mathrm{~d}, A_{\mathrm{V}} \approx 0.48 \mathrm{mag}$, and an $r^{\prime}$-band flux density of $2.3 \mu \mathrm{Jy}$ for the host galaxy. The spectrum is in fast cooling until $1.4 \mathrm{~d}$. During the fast cooling phase, $\nu_{\mathrm{a}}$ splits into two distinct frequencies: $\nu_{\mathrm{ac}}$ and $\nu_{\mathrm{sa}}$ (Granot $\&$ Sari 2002). The spectrum has the Rayleigh-Jeans shape $\left(\nu^{2}\right)$ below $\nu_{\text {ac }}$, and a slope of $\nu^{11 / 8}$ between $\nu_{\mathrm{ac}}$ and $\nu_{\mathrm{sa}}$. For the highest likelihood model, the break frequencies ${ }^{12}$ are located at $\nu_{\mathrm{ac}} \approx 2.8 \times 10^{9} \mathrm{~Hz}, \quad \nu_{\mathrm{sa}} \approx 4.9 \times 10^{9} \mathrm{~Hz}, \quad \nu_{\mathrm{c}} \approx 9.3 \times 10^{12}$, and $\nu_{\mathrm{m}} \approx 1.5 \times 10^{13} \mathrm{~Hz}$ at $1 \mathrm{~d}$ and the peak flux density is $\approx 18 \mathrm{mJy}$ at $\nu_{\mathrm{c}} . \nu_{\mathrm{m}}$ evolves as $t^{-2}$ following the jet break at $\approx 1.5 \mathrm{~d}$ to $\approx 7.7 \mathrm{GHz}$ at $50 \mathrm{~d}$, consistent with the basic considerations outlined in Section 3.2. The Compton $y$-parameter is $\approx 0.6$, indicating that cooling due to inverseCompton scattering is moderately significant.

We present histograms of the marginalized posterior density for each parameter in Figure 5. Most of our radio observations are after the transition to slow cooling, at which time $\nu_{\mathrm{a}}$ lies below the lowest radio frequency observed, resulting in degeneracies between the model parameters (Figure 6). We summarize the results of our MCMC analysis ${ }^{13}$ in Table 5.

\footnotetext{
${ }^{12} \nu_{\mathrm{c}}$ here is reported as $\sqrt{\nu_{3} \nu_{11}}$, where $\nu_{3}$ and $\nu_{11}$ are (differently normalized) expressions for the cooling frequency in the slow cooling and fast cooling regimes, respectively (Granot \& Sari 2002), such that $\nu_{3} / \nu_{11}=$ $10.87(p-0.46) e^{-1.16 p}$. We find $\nu_{3}=1.2 \times 10^{13}$ and $\nu_{11}=7.4 \times 10^{12}$ at $1 \mathrm{~d}$. 13 The upper cut-offs in the distributions of $\epsilon_{\mathrm{e}}$ and $\epsilon_{B}$ at $1 / 3$ is due to the a priori restriction $\epsilon_{\mathrm{e}}, \epsilon_{B}<1 / 3$. We note that greater values are formally allowed by the models in this case; we have elected to use $1 / 3$ as a reasonable physical restriction on these parameters.
} 

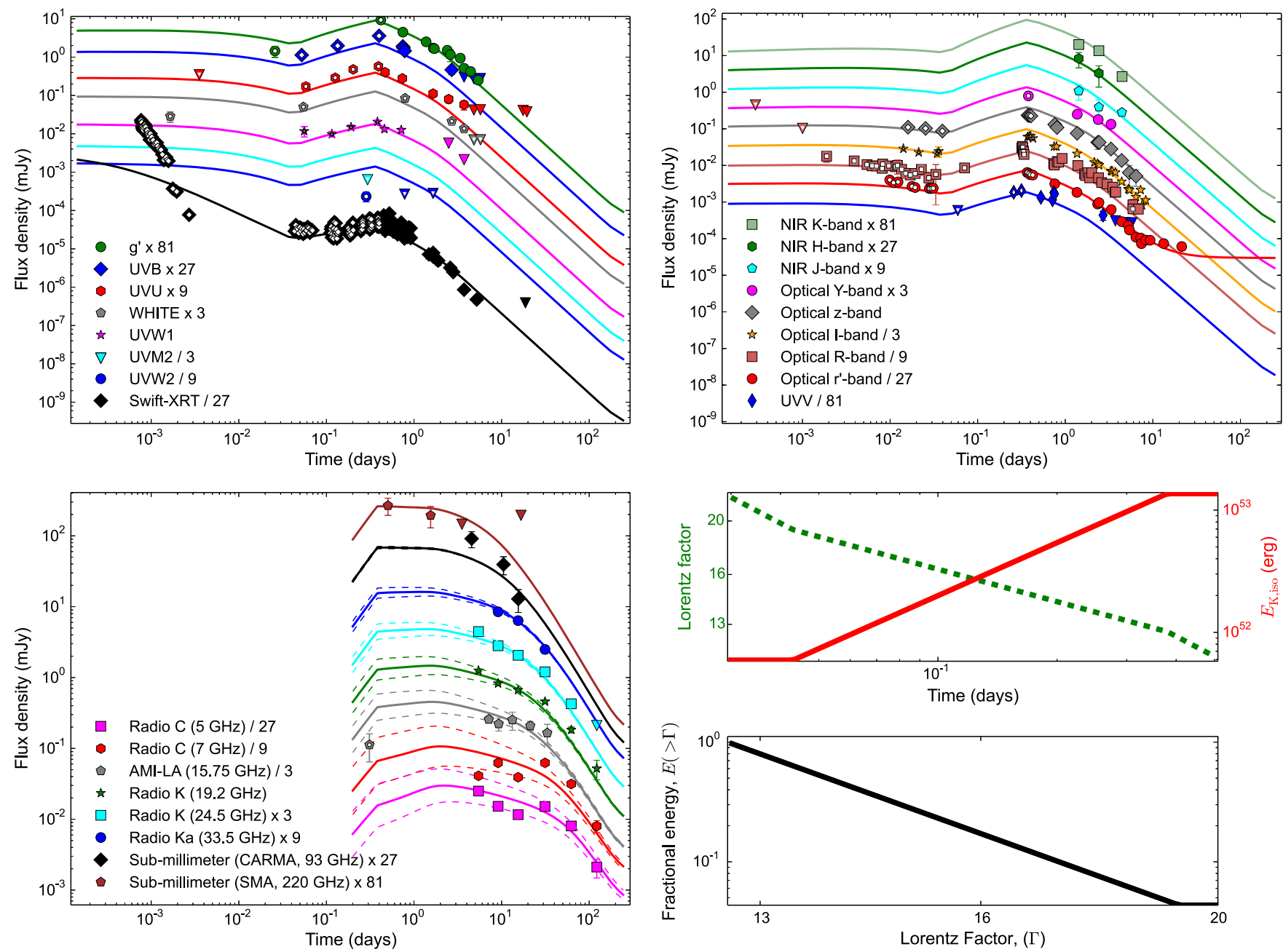

Figure 4. X-ray, UV (top left), optical (top right), and radio (bottom left) light curves of GRB 120326A in the ISM scenario, with the full afterglow model (solid lines), including energy injection before $0.4 \mathrm{~d}$. The X-ray data before $0.004 \mathrm{~d}$ is likely dominated by high-latitude prompt emission and we do not include these data in our analysis. The dashed envelopes around the radio light curves indicate the expected effect of scintillation at the $1 \sigma$ level. The data prior to the end of the rebrightening at $0.4 \mathrm{~d}$ (open symbols) are not used to determine the parameters of the forward shock in the MCMC analysis. The Swift/UVOT data in the $u v w 2$ and uvm 2 bands are strongly affected by IGM absorption and are not included in the analysis. Bottom right: blastwave Lorentz factor (green, dashed; upper sub-panel) and isotropic equivalent kinetic energy (red, solid; upper sub-panel) as a function of time, together with the energy distribution across ejecta Lorentz factors (black, solid; lower sub-panel) as determined from fitting the X-ray/UV/optical re-brightening at $0.4 \mathrm{~d}$.

Using the relation

$$
\theta_{\text {jet }}=0.17\left(\frac{E_{\mathrm{K}, \text { iso }, 52}}{n_{0}}\right)^{\frac{1}{8}}\left(\frac{t_{\text {jet }} /(1+z)}{1 \mathrm{~d}}\right)^{\frac{3}{8}}
$$

for the jet opening angle (Sari et al. 1999), and the distributions of $E_{\mathrm{K}, \text { iso }}, n_{0}$, and $t_{\text {jet }}$ from our MCMC simulations, we find $\theta_{\text {jet }}=4: 6 \pm 0: 2$. Applying the beaming correction, $E=E_{\text {iso }}\left(1-\cos \theta_{\text {jet }}\right)$, we find $E_{\gamma}=(1.0 \pm 0.1) \times 10^{50} \mathrm{erg}$ $\left(1-10^{4} \mathrm{keV}\right.$; rest frame) and $E_{\mathrm{K}}=\left(4.6_{-0.1}^{+0.2}\right) \times 10^{50} \mathrm{erg}$.

\subsection{X-Ray/UV/Optical Re-brightening}

We now consider physical explanations of the unusual rebrightening between 0.1 and 1 day observed in the X-ray, UV, and optical bands.

Scattering by dust grains in the host galaxy of GRBs has been suggested as a potential explanation for the shallow decay phase of X-ray light curves (Shao \& Dai 2007). However, this mechanism is expected to cause a significant softening of the X-ray spectrum with time (Shen et al. 2008), and cannot produce light curves that rise with time, as is observed in the case of GRB 120326A at about $0.4 \mathrm{~d}$. The bump can also not be caused by the passage of a spectral break frequency, since it occurs almost simultaneously in the X-ray, UV, and optical bands. Four remaining potential models for the bump are: (1) onset of the afterglow, (2) geometric effects due to an observer located outside the jet (off-axis scenario), (3) a density enhancement in the circumburst environment, and (4) a refreshed shock (energy injection scenario). We now explore these possibilities in turn.

\subsubsection{Afterglow Onset}

In this scenario, the peak of the light curve emission corresponds to emission from a RS due to deceleration of the ejecta by the surrounding material. The ejecta are assumed to be composed of a conical shell segment at a single Lorentz factor. The RS light curves before and after the deceleration time depend on the properties of the environment (ISM or wind) and the ejecta. RS light curves for a constant density environment depend upon whether the ejecta shell is thick 

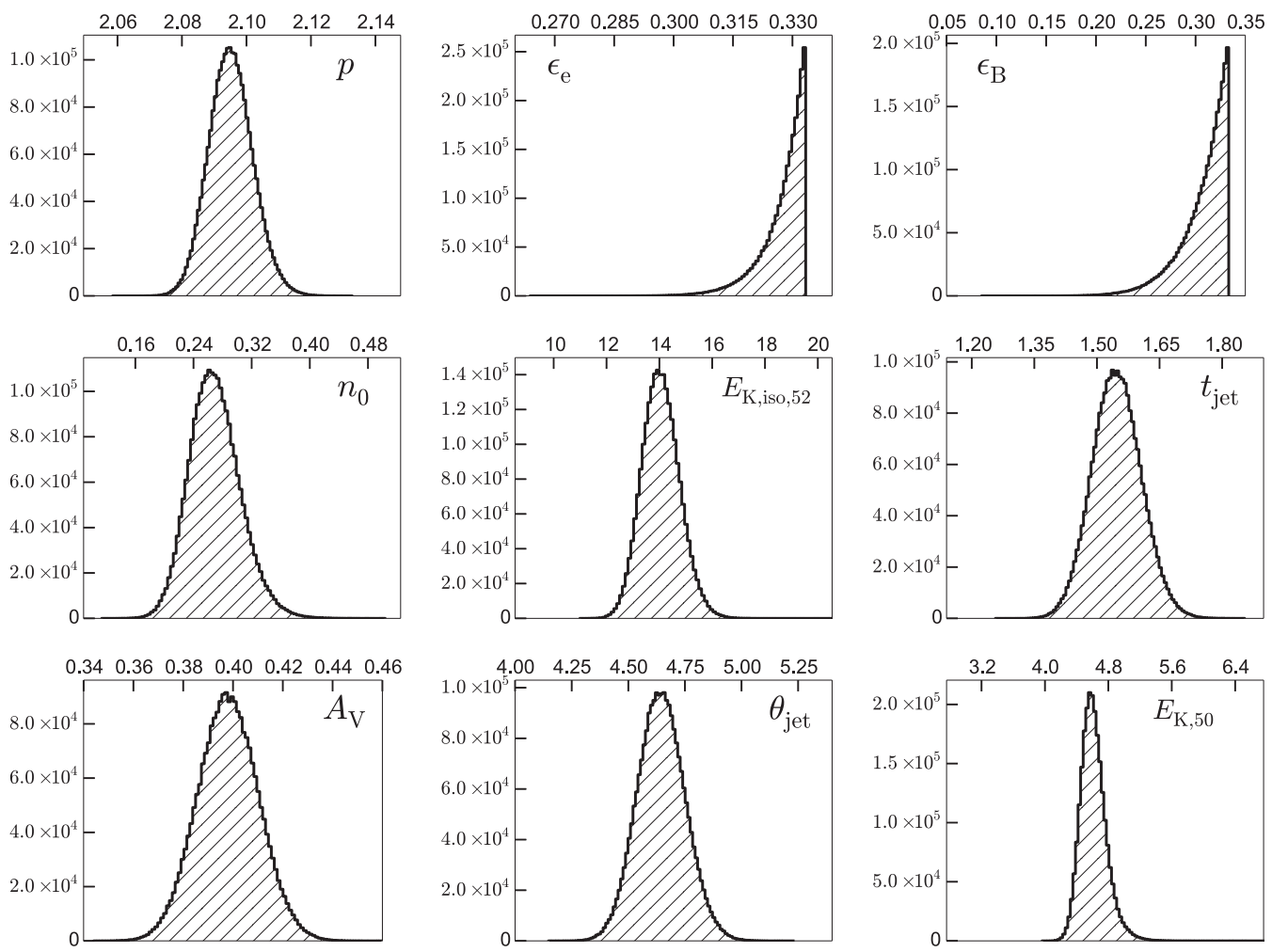

Figure 5. Posterior probability density functions for the physical parameters of GRB 120326A in the ISM model from MCMC simulations. We have restricted $\epsilon_{\mathrm{e}}<1 / 3$ and $\epsilon_{B}<1 / 3$. (An extended version of this figure is available.)
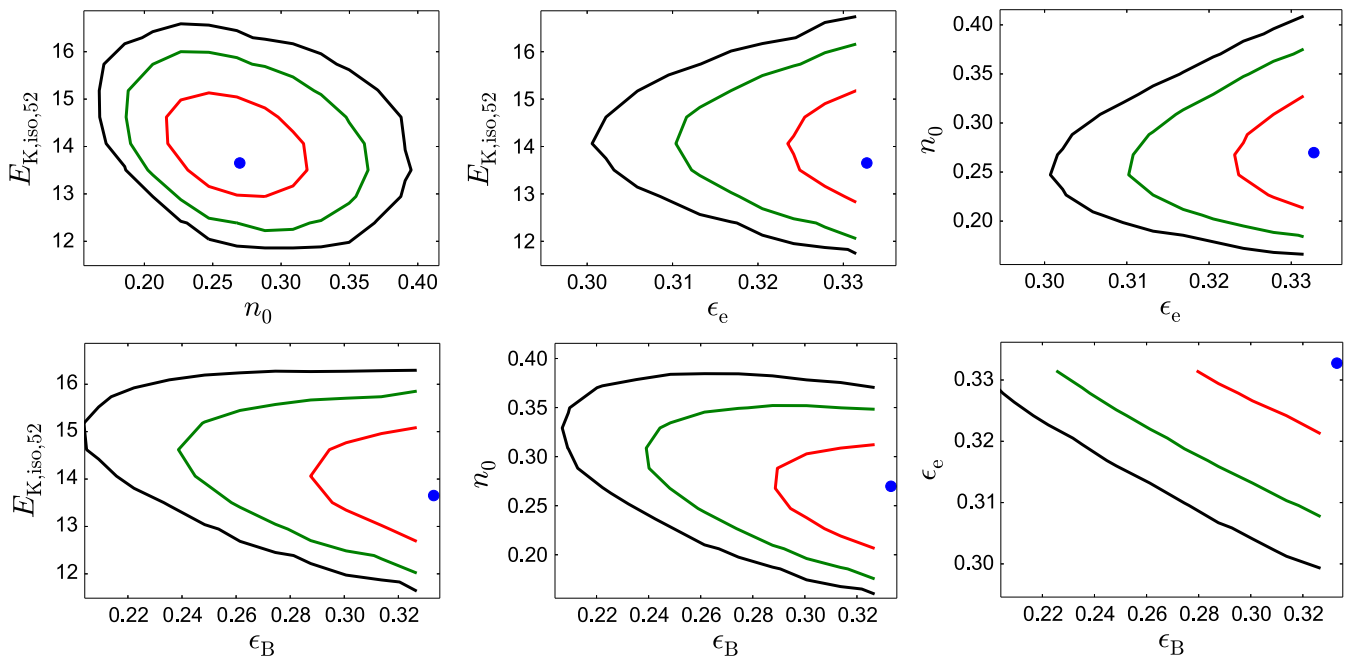

Figure 6. $1 \sigma$ (red), $2 \sigma$ (green), and $3 \sigma$ (black) contours for correlations between the physical parameters, $E_{\mathrm{K}, \text { iso }}, n_{0}, \epsilon_{\mathrm{e}}$, and $\epsilon_{B}$ for GRB $120326 \mathrm{~A}$, in the ISM model from Markov chain Monte Carlo simulations, together with the maximum-likelihood model (blue dot). We have restricted $\epsilon_{\mathrm{e}}<{ }^{1 / 3}$ and $\epsilon_{B}<1 / 3$. See the on-line

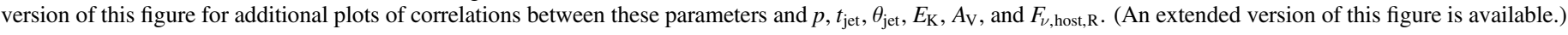

$\left(\Delta_{0}>l / 2 \Gamma_{0}^{8 / 3}\right)$ or thin $\left(\Delta_{0}>l / 2 \Gamma_{0}^{8 / 3}\right)$, where $\Delta_{0}$ is the initial shell width, $\Gamma_{0}$ is the initial Lorentz factor of the ejecta and $l=\left(3 E / 4 \pi n_{0} m_{\mathrm{p}} c^{2}\right)^{1 / 3}$ is the Sedov length (Kobayashi 2000). Similarly, when the circumburst medium has a wind-like profile, the light curves again depend on whether $\Delta_{0}>E / 4 \pi A c^{2} \Gamma_{0}^{4}$ (thick shell) or $\Delta_{0}<E / 4 \pi A c^{2} \Gamma_{0}^{4}$ (thin shell; Kobayashi \& Zhang 2003).

In the thick shell case, the RS crosses the ejecta in a time comparable to the burst duration, $t_{\gamma}$, and the light curves at all frequencies are expected to peak at that timescale. In the case of
GRB $120326 \mathrm{~A}, T_{90}$ in the Swift/BAT band is $\approx 27 \mathrm{~s}$, which is much shorter than the observed time at which the light curves peak, $t_{\text {peak }} \approx 0.4 \mathrm{~d}$. Thus, the re-brightening cannot be explained by RS emission in the thick shell case.

In the thin shell case, temporal separation is expected between the GRB itself and the peak of the RS, which occurs when the RS crosses the shell at $t_{\gamma}=90 E_{\mathrm{K}, \text { iso }, 52}^{1 / 3} n_{0}^{-1 / 3} \Gamma_{0,100}^{-8 / 3} \mathrm{~s}$ (ISM environment; Kobayashi 2000) or $t=2.9 \times 10^{3}$ $(1+z) E_{\mathrm{K}, \text { iso }, 52} \Gamma_{0,100}^{-4} A_{*}^{-1} \mathrm{~s} \quad$ (wind environment; Zou et al. 2005). A RS peak at $0.4 \mathrm{~d}$ would imply a rather low 
Table 5

Energy Injection in GRB Afterglows

\begin{tabular}{|c|c|c|c|c|}
\hline GRB & $100418 \mathrm{~A}$ & $100901 \mathrm{~A}$ & $120326 \mathrm{~A}$ & $120404 \mathrm{~A}$ \\
\hline Redshift, $z$ & 0.6235 & 1.408 & 1.798 & 2.876 \\
\hline$T_{90}(\mathrm{~s})$ & $8.0 \pm 2.0$ & $439 \pm 33$ & $11.8 \pm 1.8$ & $38.7 \pm 4.1$ \\
\hline \multirow{2}{*}{$E_{\gamma, \text { iso }}\left(\mathrm{erg} ; 1-10^{4} \mathrm{keV}\right.$, rest frame $)$} & $9.9_{-3.4}^{+6.3} \times 10^{50}$ & $(8 \pm 1) \times 10^{52}$ & $(3.2 \pm 0.1) \times 10^{52}$ & $(9 \pm 4) \times 10^{52}$ \\
\hline & \multicolumn{4}{|c|}{ Best-fit model $^{\mathrm{a}}$} \\
\hline Section in text & 6.1 .2 & 6.2 .2 & 5.1 & 6.3 .2 \\
\hline$\epsilon_{\mathrm{e}}$ & 0.12 & 0.33 & 0.33 & 0.27 \\
\hline$\epsilon_{B}$ & $1.1 \times 10^{-2}$ & 0.32 & 0.33 & 0.16 \\
\hline$n_{0}\left(\mathrm{~cm}^{-3}\right)$ & 1.4 & $3.2 \times 10^{-3}$ & 0.27 & $2.8 \times 10^{2}$ \\
\hline$E_{\mathrm{K}, \text { iso }}\left(10^{52} \mathrm{erg}\right)$ & 3.36 & 29.7 & 13.7 & 12.3 \\
\hline$A_{\mathrm{V}}(\mathrm{mag})$ & $\lesssim 0.1$ & 0.1 & 0.40 & 0.13 \\
\hline$t_{\text {jet }}$ (days) & 16.9 & 0.96 & 1.54 & $6.6 \times 10^{-2}$ \\
\hline$E_{\mathrm{K}, \text { iso,initial }}(\mathrm{erg})$ & $1.0 \times 10^{50}$ & $5.4 \times 10^{52}$ & $3.1 \times 10^{51}$ & $4.4 \times 10^{51}$ \\
\hline \multirow[t]{2}{*}{$E_{\mathrm{K}, \text { initial }}(\mathrm{erg})$} & $6.3 \times 10^{48}$ & $3.8 \times 10^{49}$ & $8.7 \times 10^{48}$ & $6.4 \times 10^{48}$ \\
\hline & \multicolumn{4}{|c|}{ MCMC Results $^{\mathrm{a}}$} \\
\hline$p$ & $2.13_{-0.01}^{+0.02}$ & $2.05 \pm 0.01$ & $2.095 \pm 0.007$ & $2.07 \pm 0.02$ \\
\hline$\epsilon_{\mathrm{e}}$ & $1.2_{-0.2}^{+0.3} \times 10^{-1}$ & $0.30_{-0.05}^{+0.03}$ & $0.329_{-0.007}^{+0.003}$ & $0.27_{-0.05}^{+0.04}$ \\
\hline$\epsilon_{B}$ & $9.4_{-3.1}^{+3.5} \times 10^{-3}$ & $0.12_{-0.08}^{+0.12}$ & $0.31_{-0.03}^{+0.01}$ & $0.13_{-0.08}^{+0.12}$ \\
\hline$n_{0}\left(\mathrm{~cm}^{-3}\right)$ & $1.6_{-0.3}^{+0.4}$ & $7.2_{-3.1}^{+6.1} \times 10^{-3}$ & $0.27_{-0.03}^{0.04}$ & $3.5_{-1.8}^{+4.9} \times 10^{2}$ \\
\hline $\log \left(n_{0}\right)^{\mathrm{b}}$ & $0.22 \pm 0.09$ & $-2.1_{-0.2}^{+0.3}$ & $-0.57 \pm 0.06$ & $2.5_{-0.3}^{+0.4}$ \\
\hline$E_{\mathrm{K}, \text { iso }}\left(10^{52} \mathrm{erg}\right)$ & $3.6_{-0.7}^{+1.0}$ & $31.1_{-4.3}^{+8.6}$ & $14.0 \pm 0.07$ & $13.3_{-2.0}^{+3.5}$ \\
\hline$A_{\mathrm{V}}(\mathrm{mag})$ & $\lesssim 0.1$ & $0.09 \pm 0.01$ & $0.40 \pm 0.01$ & $0.13 \pm 0.01$ \\
\hline
\end{tabular}

Notes.

${ }^{a}$ Note that the parameters for the best-fit model may differ slightly from the results from the MCMC analysis. This is because the former is the peak of the likelihood distribution (and is appropriate for generating model light curves), while the latter is summarized here as $68 \%$ credible intervals about the median of the marginalized posterior density functions for each parameter. The posterior density serves as our best estimate for the value of each parameter, and may be asymmetric about the value corresponding to the highest-likelihood model.

${ }^{\mathrm{b}}$ In instances where the measured value of $n_{0}$ spans more than about a factor of $2, \log n_{0}$ is a more meaningful quantity. We therefore report both $n_{0}$ and $\log n_{0}$ for all cases.

burst Lorentz factor of $\Gamma_{0} \approx 17$. Additionally, the light curves at all frequencies would be expected to rise until $t_{\gamma}$ and decline thereafter (except above $\nu_{\mathrm{c}}$ where the flux is constant until $t_{\gamma}$ and disappears after $t_{\gamma}$ ). The observed $R$-band light curve, on the other hand, declines between 100 and $500 \mathrm{~s}$, followed by a flat segment until $3500 \mathrm{~s}$, followed by the rise into the bump at $0.4 \mathrm{~d}$. This combination of a flat portion followed by a rise cannot be explained as being due to an RS.

Thus the onset of the afterglow does not provide a viable explanation for the re-brightening regardless of density profile, and whether the ejecta are in the thick- or thin-shell regime.

\subsubsection{Off-axis Model}

We now investigate the re-brightening in the context of viewing geometry effects. We consider a jet with an opening half-angle, $\theta_{\text {jet }}$, viewed from an angle $\theta_{\text {obs }}$. While small offsets in the observer's viewing angle relative to the jet axis do not cause significant changes in the light curves provided that $\theta_{\text {obs }} \lesssim \theta_{\text {jet }}$, it is possible to obtain a rising light curve when $\theta_{\text {obs }} \gtrsim 2 \theta_{\text {jet }}$ (Granot et al. 2001; 2002). In this case, the time of the peak of the light curve, $t_{\mathrm{p}}$ is related to the jet break time for an on-axis viewer as $t_{\mathrm{p}} \approx(5+2 \ln \Theta) \Theta^{2} t_{\text {jet }} \gtrsim 5 t_{\text {jet }}$, where $\Theta \equiv \theta_{\text {obs }} / \theta_{\text {jet }}-1 \gtrsim 1$ (Granot et al. 2002). This implies that for a light curve that peaks at $\approx 0.4 \mathrm{~d}$, the on-axis jet break time must have occurred earlier, at $\approx 2 \mathrm{hr}$.

In this case, the radio light curves are expected to rise until the observer's line of sight enters the beaming cone of the jet and then approximately converge with the predicted on-axis light curves after $0.4 \mathrm{~d}$. After the jet break, the flux density declines as $t^{-1 / 3}$ for $\nu<\nu_{\mathrm{m}}$ and as $t^{-p}$ for $\nu>\nu_{\mathrm{m}}$ (for an onaxis observer; Sari et al. 1999). Thus, the radio light curves should be declining at all frequencies after $0.4 \mathrm{~d}$. However, the flux density at the $15.75 \mathrm{GHz}$ AMI-LA band rises from $(0.34 \pm$ $0.14) \mathrm{mJy}$ at $0.31 \mathrm{~d}$ to $(0.77 \pm 0.08) \mathrm{mJy}$ at $7.15 \mathrm{~d}$, which is 
inconsistent with this expectation. Further, relativistic beaming of the prompt radiation within the jet cone would typically result in an orphan afterglow (e.g., Huang et al. 2002) or require a large intrinsic energy for the GRB to be visible off-axis (e.g., Margutti et al. 2010a). This argues against the off-axis model as an explanation for the X-ray/UV/optical re-brightening.

\subsubsection{Density Enhancement}

If the blastwave encounters an enhancement in the local density as it propagates into the circumburst environment, an increase in the flux density is expected. Bumps in afterglow light curves have been ascribed to this phenomenon in the past (e.g., Berger et al. 2000; Lazzati et al. 2002; Schaefer et al. 2003). However, the flux density at frequencies above $\nu_{\mathrm{c}}$ (for slow-cooling spectra) and above $\nu_{\mathrm{m}}$ (for fast cooling spectra) are insensitive to variations in the circumburst density (Nakar \& Granot 2007). In the case of GRB 120326A, a rebrightening is observed both in the UV/optical and in the $\mathrm{X}$-rays. The X-rays are located above both $\nu_{\mathrm{c}}$ and $\nu_{\mathrm{m}}$ in the $p \approx 2.1$ (fast cooling) model. Thus in our favored afterglow model, the X-ray re-brightening cannot be due to a density enhancement. Additionally, Geng et al. (2014) find that a density jump in the circumburst medium usually does not lead to a significant rebrightening, even when emission from the resulting RS is included.

\subsubsection{Energy Injection Model}

An alternative model for a re-brightening of the afterglow is the injection of energy into the blastwave shock due to prolonged central engine activity, deceleration of a Poynting flux dominated outflow, or the presence of substantial ejecta mass (and hence kinetic energy) at low Lorentz factors (Dai \& Lu 1998; Rees \& Meszaros 1998; Kumar \& Piran 2000; Sari \& Mészáros 2000; Zhang \& Mészáros 2001, 2002; Granot \& Kumar 2006; Zhang et al. 2006; Dall'Osso et al. 2011; Uhm et al. 2012). This mechanism has been invoked to explain plateaus in the observed light curves of a large fraction of GRB X-ray afterglows (Nousek et al. 2006; Liang et al. 2007; Yu \& Dai 2007; Margutti et al. 2010a; Hascoët et al. 2012; Xin et al. 2012). In this section, we explore the energy-injection model as a possible explanation for the X-ray/UV/optical re-brightening at $0.4 \mathrm{~d}$.

Models involving a transfer of energy from braking radiation of millisecond magnetars into the forward shock predict plateaus in X-ray light curves but do not generally lead to a re-brightening. In particular, they require an injection luminosity, $L \propto t^{q}$ (corresponding to an increase in the blastwave energy with time as $E \propto t^{1+q}$ ), with $q \leqslant 0$. In our $p \approx 2.1$ model, the X-rays are located above the cooling frequency. In this regime, the flux density above $\nu_{\mathrm{c}}$ is $F_{\nu>\nu_{\mathrm{c}}} \propto$ $E_{\mathrm{K}, \text { iso }}^{(2+p) / 4} t^{(2-3 p) / 4}$ (Granot \& Sari 2002). For $p \approx 2.1$, this reduces to $F_{\nu>\nu_{\mathrm{c}}} \propto E_{\mathrm{K}, \text { iso }}^{1.03} t^{-1.1}$. During energy injection, $E \propto t^{m}$, such that $F_{\nu>\nu_{\mathrm{c}}} \propto t^{1.03 m-1.1}$. The steep rise $\left(\alpha_{1}=0.85 \pm 0.19\right.$; Section 3.1) requires $m=1.89 \pm 0.18$ or $q \equiv m-1$ $=0.89 \pm 0.18$. Energy injection due to spin-down or gravitational wave radiation from a magnetar is expected to provide at best a constant luminosity $(q \leqslant 0)$. Thus the observed rebrightening cannot be explained by energy injection from a magnetar. In the case of energy injection due to fall-back accretion onto a black hole, the expected accretion rate is
$\dot{M} \propto t^{-5 / 3}$ (Phinney 1989), which is also insufficient to power the observed plateaus. Similarly, central engine activity is usually associated with flaring behavior in X-ray (Burrows et al. 2005a; Fan \& Wei 2005; Falcone et al. 2006; Perna et al. 2006; Proga \& Zhang 2006; Zhang et al. 2006; Chincarini et al. 2007, 2010; Margutti et al. 2010b, 2011a; Bernardini et al. 2011) and optical ( $\mathrm{Li}$ et al. 2012) light curves, but it involves shorter characteristic time scales $(\Delta t / t \ll 1)$ than observed for GRB $120326 \mathrm{~A}$ at $0.4 \mathrm{~d}$.

In the standard afterglow model, the ejecta are assumed to have a single Lorentz factor. We now relax this assumption and consider models including a distribution of Lorentz factors in the ejecta as a possible explanation for the late re-brightening in GRB 120326A. Provided they are released over a time range small compared to the afterglow timescale, the ejecta arrange themselves in homologous expansion with the Lorentz factors monotonically increasing with distance from the source (Kumar \& Piran 2000). We follow the formalism of Sari \& Mészáros (2000), where the ejecta are assumed to possess a continuous distribution of Lorentz factors such that a mass, $M(>\Gamma) \propto \Gamma^{-s}$ is moving with Lorentz factors greater than $\Gamma$ with corresponding energy, $E(>\Gamma) \propto \Gamma^{-s+1}$ down to some minimum Lorentz factor, $\Gamma_{\min }$ (also known as the "massiveejecta model"). Additionally, we posit a maximum Lorentz factor for this distribution, $\Gamma_{\max }$, corresponding to the Lorentz factor of the blastwave $\Gamma_{\mathrm{BW}}$ at the onset of energy injection. In this model, there is a gap between the blastwave, which is powered by an initial shell of fast-moving ejecta, and the leading edge of the remaining ejecta traveling at $\Gamma_{\max }$. When these slower ejecta shells catch up with the blastwave at a time when $\Gamma_{\mathrm{BW}}(t) \approx \Gamma_{\max }$, they begin depositing energy into the blastwave, and energy injection commences. This proceeds until the lowest energy ejecta located at $\Gamma_{\text {min }}$ have transferred their energy to the blastwave, and the subsequent evolution proceeds like a standard afterglow powered by a blastwave with increased energy. The Lorentz factor of the blastwave at the end of energy injection is therefore $\Gamma_{\min }$, which can be determined from modeling the subsequent afterglow evolution and invoking the standard hydrodynamical framework.

For an ambient medium with a density profile, $n \propto r^{-k}$, the energy of the blastwave increases as $E \propto t^{m}$ during the period of energy injection, where ${ }^{14} m=\frac{(3-k)(s-1)}{7+s-2 k}$. For a constant density medium $(k=0)$ this translates to $m=\frac{3(s-1)}{7+s}$, which is bounded $(0<m<3$ for $s \in[1, \infty)$ ) while for a wind-like environment $(k=2)$ we have $m=\frac{s-1}{s-3}$, which is also bounded $(0<m<1$ for $s \in[1, \infty))$.

For GRB 120326A, the optical and X-ray light curves are located above both $\nu_{\mathrm{m}}$ and $\nu_{\mathrm{c}}$ and the spectrum is fast cooling $\left(\nu_{\mathrm{c}}<\nu_{\mathrm{m}}\right.$; Section 5.1). In this regime, the flux density depends on the blastwave energy and time as, $F_{\nu} \propto E^{(2+p) / 4} t^{(2-3 p) / 4}$. Writing $E=E_{0}\left(t / t_{0}\right)^{m}$ for the energy injection episode, this implies $F_{\nu} \propto t^{[(2+p) m+2-3 p] / 4} \propto t^{m-1}$, for $p=2$. Since the optical and X-ray light curves rise with temporal index, $\alpha \approx 0.6$ (Section 3.1) and $p \approx 2$ (Section 5.1), this implies $m \approx 1.6$, which corresponds to $s=\frac{7 m+3}{3-m} \approx 10$.

For a detailed analysis, we use the afterglow parameters for the maximum likelihood model listed in Section 5.1 as our starting model (which explains all available multi-wavelength

\footnotetext{
14 Other authors have defined this in terms of luminosity, with $L \propto t^{-q}$, or $E \propto t^{1-q}$. Thus $m \equiv 1-q$.
} 
data after the re-brightening). We then reduce the energy at earlier times as a power law with time,

$$
E_{\mathrm{K}, \text { iso }}(t)= \begin{cases}E_{\mathrm{K}, \text { iso }, \mathrm{f}}, & t \geqslant t_{0} \\ E_{\mathrm{K}, \text { iso }, \mathrm{f}}\left(\frac{t}{t_{0}}\right)^{m}, & t_{1}<t<t_{0} \\ E_{\mathrm{K}, \text { iso }, \mathrm{i}} \equiv E_{\mathrm{K}, \text { iso }, \mathrm{f}}\left(\frac{t_{1}}{t_{0}}\right)^{m}, & t \leqslant t_{1},\end{cases}
$$

where $E_{\mathrm{K} \text {,iso,i }}$ and $E_{\mathrm{K} \text {,iso,f }}$ are the initial and final energy of the blastwave, respectively, with the latter fixed to the value determined by our MCMC analysis of the data at $\geqslant 0.4 \mathrm{~d}$. Energy injection into the blastwave at the rate $E(t) \propto t^{m}$ begins at $t_{1}$ and ends at $t_{0}$, with $t_{0}, t_{1}$, and $m$ being free parameters in this model. We compute the spectrum at each time according to the instantaneous energy in the blastwave, adjusting the parameters $t_{0}$ and $t_{1}$ by hand until the resulting light curves match the observations.

We find that the X-ray and optical light curves can be modeled well by a single period of energy injection, with $E_{\mathrm{K} \text {,iso }}=1.4 \times 10^{53} \operatorname{erg}(t / 0.38 \mathrm{~d})^{1.44}$ between $t_{1}=4.3 \times 10^{-2} \mathrm{~d}$ and $t_{0}=0.38 \mathrm{~d}$ (Figure 4). In this model, the blastwave energy at the start of energy injection is $E_{\mathrm{K}, \text { iso, } \mathrm{i}} \approx 5.9 \times 10^{51}$ erg. Thus $\approx 96 \%$ of the final energy of the blastwave is injected during this episode. In comparison, $E_{\gamma \text {, iso }}=$ $(3.15 \pm 0.12) \times 10^{52} \mathrm{erg}$ (Section 3$)$. The blastwave Lorentz factor can be computed from the expression $\Gamma=$ $3.65 E_{\mathrm{K}, \text { iso }, 52}{ }^{1 / 8} n_{0}^{-1 / 8} t^{-3 / 8}(1+z)^{3 / 8}$ (Granot \& Sari 2002) and is $\Gamma \approx 19$ at the start of energy injection, decreasing to $\Gamma \approx 13$ at $0.38 \mathrm{~d}$. Interpreted in the context of the massiveejecta model, the energy injection rate of $m=1.44$ would imply $s=8.4$, corresponding to an ejecta distribution with $E(>\Gamma) \propto \Gamma^{1-s} \propto \Gamma^{-7.4}$ for $13 \lesssim \Gamma \lesssim 19$.

\section{PANCHROMATIC RE-BRIGHTENING EPISODES IN OTHER GRBS}

Whereas individual flattening or re-brightening episodes have been seen both in optical and X-ray observations of GRB afterglows (Liang et al. 2007; Li et al. 2012), simultaneous rebrightening of the afterglow in multiple bands spanning both the optical and the X-rays as seen in GRB 120326A is quite rare. The X-ray and $R$-band light curves of GRB 970508 exhibited a bump at around $60 \mathrm{ks}$, lasting for about $100 \mathrm{ks}$ (Piro et al. 1998). Multiple episodes of flux variations of $\approx 30 \%$ on timescales of $\Delta t / t \approx 1$ between 1 and $10 \mathrm{~d}$ since the burst were detected superposed on a power-law decline in multi-band X-ray and $B V R I$-band data for GRB 030329 (Lipkin et al. 2004), while de Ugarte Postigo et al. (2007) detected an X-ray and optical re-brightening in GRB 050408 around $260 \mathrm{ks}$. The short burst GRB 050724 had a large re-brightening at around $42 \mathrm{ks}$, simultaneous with an optical flare (Berger et al. 2005; Malesani et al. 2007; Margutti et al. 2011b). GRB 060206 exhibited a simultaneous X-ray and optical rebrightening at $3.8 \mathrm{ks}$ (Monfardini et al. 2006; Liu et al. 2008). GRB 070311 exhibited a re-brightening at $\approx 100 \mathrm{ks}$ in the $\mathrm{X}$-rays and in $R$-band, but the peak in the X-rays is not wellsampled and does not appear to have occurred at the same time in the two bands (Guidorzi et al. 2007). Covino et al. (2008) report a simultaneous $\mathrm{X}$-ray and $R J H K$-band re-brightening for GRB $071010 \mathrm{~A}$ at $\approx 52 \mathrm{ks}$. GRB 060614 exhibited a prominent brightening in the $U B V R$-bands simultaneous with an X-ray plateau (Mangano et al. 2007). Similarly, the $z \approx 6$ GRB $120521 \mathrm{C}$ exhibited an X-ray plateau at the same time as a bump in the $z$-band light curve (Laskar et al. 2014). GRB 081028 was one of the first events with a well-sampled rising X-ray light curve following the steep decay phase with a concomitant optical re-brightening (Margutti et al. 2010a). An $\mathrm{X}$-ray re-brightening simultaneous with a re-brightening in Swift/UVOT observations was seen for GRB 100418A and interpreted as energy injection by Marshall et al. (2011). Gorbovskoy et al. (2012) report a prominent multi-band rebrightening in GRB 100901A, whose X-ray light curve is remarkably similar to that of GRB 120326A. GRB 120404A was found to exhibit a strong re-brightening in the optical and NIR, although X-ray observations around the time of the rebrightening are sparse as this time fell within an orbital gap of Swift (Guidorzi et al. 2014).

Of these instances of X-ray or optical re-brightening events seen in GRB afterglow light curves, we select those objects with multi-band (at least X-ray and optical) data sets that exhibit a simultaneous re-brightening in both X-rays (following the steep decay phase) and optical/NIR. Since radio observations are vital for constraining the physical parameters, we restrict our sample to events with radio detections: GRBs 100418A, 100901A, and 120404A. We perform a full multiwavelength afterglow analysis for these events and compare our results with those for GRB 120326A in Section 7.

\subsection{GRB $100418 A$}

\subsubsection{GRB Properties and Basic Considerations}

GRB 100418A was detected and localized by the Swift BAT on 2010 April 18 at 21:10:08 UT (Marshall et al. 2010). The burst duration is $T_{90}=8.0 \pm 2.0 \mathrm{~s}$, with a fluence of $F_{\gamma}=(3.4 \pm 0.5) \times 10^{-7} \mathrm{erg} \mathrm{cm}^{-2} \quad(15-150 \mathrm{keV} \quad$ observer frame; Marshall et al. 2011). The afterglow was detected in the X-ray and optical bands by XRT, UVOT, and various ground-based observatories (Filgas et al. 2010; Siegel \& Marshall 2010; Updike et al. 2010c), as well as in the radio by WSRT, VLA, the Australia Telescope Compact Array (ATCA), and the Very Long Baseline Array (VLBA; Chandra \& Frail 2010b; Moin et al. 2010; van der Horst et al. 2010a). Spectroscopic observations with the ESO Very Large Telescope (VLT) yielded a redshift of $z=0.6235$ (Antonelli et al. 2010). By fitting the $\gamma$-ray spectrum with a Band function, Marshall et al. (2011) determined the isotropic equivalent $\gamma$-ray energy of this burst to be $E_{\gamma, \text { iso }}=\left(9.9_{-3.4}^{+6.3}\right) \times 10^{50} \mathrm{erg}$ (1-10 $0^{4} \mathrm{keV}$; rest frame).

The X-ray light curve for this burst gently rises to $0.7 \mathrm{~d}$, while the optical light curves exhibit a slow brightening at the same time. Both the X-ray and optical light curves break into a powerlaw decline following the peak. Using XRT and UVOT Whiteband observations, Marshall et al. (2011) find that the X-ray and optical bands are located on the same part of the synchrotron spectrum after the peak at $0.7 \mathrm{~d}$. By fitting the X-ray and optical decline rates and X-ray-to-optical SED, they find that both $\nu_{\mathrm{m}}$ and $\nu_{\mathrm{c}}$ are located below the optical, requiring $p \approx 2.3$. In this regime, the light curves are insensitive to the density profile of the circumburst environment. In the absence of radio data, Marshall et al. (2011) assume an ISM model and determine that the non-detection of a jet break out to $23 \mathrm{~d}$ requires a high beaming-corrected energy, $E_{\mathrm{K}} \geqslant 3 \times$ 
Table 6

Swift/UVOT Observations of GRB 100418A

\begin{tabular}{lccccc}
\hline \hline $\begin{array}{l}t-t_{0} \\
(\text { days })\end{array}$ & Filter & $\begin{array}{c}\text { Frequency } \\
(\mathrm{Hz})\end{array}$ & $\begin{array}{c}\text { Flux Density } \\
(\mu \mathrm{Jy})\end{array}$ & $\begin{array}{c}\text { Uncertainty } \\
(\mu \mathrm{Jy})\end{array}$ & $\begin{array}{c}\text { Detection? } \\
(1=\mathrm{Yes})\end{array}$ \\
\hline 0.00188 & White & $8.64 \mathrm{e}+14$ & 11.5 & 2.47 & 1 \\
0.00499 & White & $8.64 \mathrm{e}+14$ & 10.6 & 2.81 & 1 \\
0.00591 & $u$ & $8.56 \mathrm{e}+14$ & 8.41 & 2.74 & 0 \\
0.00753 & $b$ & $6.92 \mathrm{e}+14$ & 74.7 & 22.3 & 0 \\
0.011 & White & $8.64 \mathrm{e}+14$ & 10.8 & 3.25 & 1 \\
\hline
\end{tabular}

(This table is available in its entirety in machine-readable form.)

$10^{52} E_{\mathrm{K}, \text { iso }, 54}^{3 / 4} n_{0}^{1 / 4}$. They investigate the off-axis model, and compute an intrinsic burst duration for an on-axis observer of $T_{90}^{\text {on }}=\mathcal{D}\left(\theta=\theta_{\text {obs }}-\theta_{\text {jet }}\right) / \mathcal{D}(\theta=0) T_{90} \approx 10 \mathrm{~ms}$, where $\mathcal{D} \equiv\left[\gamma_{0}\left(1-\beta_{0} \cos \theta\right)\right]^{-1}$ is the Doppler factor, $\theta_{\text {obs }}$ is the offaxis viewing angle, $\theta_{\text {jet }}$ is the jet opening half-angle, and $T_{90}$ is the observed burst duration. Based on this short on-axis $T_{90}^{\text {on }}$ and large intrinsic energy output, they argue against the off-axis scenario.

Marshall et al. (2011) also consider the energy injection model. For the magnetar model involving injection of energy into the blastwave via Poynting flux, they find $q=-0.23_{-0.14}^{+0.13}$, which is marginally consistent with the $q=0$ case. For models with a Lorentz factor distribution, they use their measured value of $q$ to calculate $s=6.4_{-1.0}^{+1.3}$ (ISM model), or $s<0$ (wind model, which is unphysical). They argue that since the ISM value is too different from the value $s \approx 2.5$ indicated by X-ray plateaus in Swift observations (Nousek et al. 2006), this mode of energy injection is unlikely.

Moin et al. (2013) reported multi-wavelength radio observations of GRB 100418A from 5.5 to $9.0 \mathrm{GHz}$ from ATCA, VLA and VLBA. Using upper limits on the expansion rate of the ejecta derived from the VLBA observations, they report an upper limit on the average ejecta Lorentz factor of $\Gamma \lesssim 7$ and an upper limit on the ejecta mass of $M_{\mathrm{ej}} \lesssim 0.5 \times 10^{-3} M_{\odot}$. They use this limit on $\Gamma$ to suggest that the contribution of lowLorentz factor ejecta to the blastwave energy must be negligible during the period of energy injection, and that a separate injection mechanism is required. We include their radio observations in our analysis (Section 6.1.2) and address the constraints derived from the VLBA data in Section 6.1.3.

Marshall et al. (2011) fit multi-band UVOT and XRT spectra at three different epochs $\left(7 \times 10^{-2}, 0.8\right.$, and $\left.7.3 \mathrm{~d}\right)$ and found that a single power law with spectral index $\beta \approx-1.0$ fit all three epochs well. We follow the procedures outlined by Evans et al. (2007); (2009), and Margutti et al. (2013) to obtain time-resolved XRT spectra for this burst. The spectra are well fit by an absorbed single power-law model with $N_{\mathrm{H}, \mathrm{Gal}}=4.78 \times 10^{20} \mathrm{~cm}^{-2} \quad$ (Kalberla et al. 2005), and intrinsic hydrogen column $N_{\mathrm{H} \text {,int }}=\left(0.7_{-0.7}^{+1.5}\right) \times 10^{21} \mathrm{~cm}^{-2}$, with $\Gamma=3.35 \pm 0.09\left(85-130 \mathrm{~s} ; \chi^{2}=1.74\right.$ for 123 degrees of freedom) and $\Gamma=3.9 \pm 0.3\left(130 \mathrm{~s}-180 \mathrm{~s} ; \chi^{2}=0.97\right.$ for 55 degrees of freedom) during the early and late WT-mode steep decay, respectively, followed by $\Gamma_{\mathrm{X}}=2.08 \pm 0.18$ $\left(2.3 \times 10^{-3} \mathrm{~d}-1.8 \mathrm{~d} ; \chi^{2}=0.75\right.$ for 79 degrees of freedom $)$ during the plateau phase, and $\Gamma_{\mathrm{X}}=1.81 \pm 0.20(1.8-34.5 \mathrm{~d}$; $\chi^{2}=0.70$ for 89 degrees of freedom) for the remainder of the observations. We obtain a flux light curve in the $0.3-10 \mathrm{keV}$ XRT band following the methods reported by Margutti et al. (2013), which we convert to a flux density light curve at $1 \mathrm{keV}$

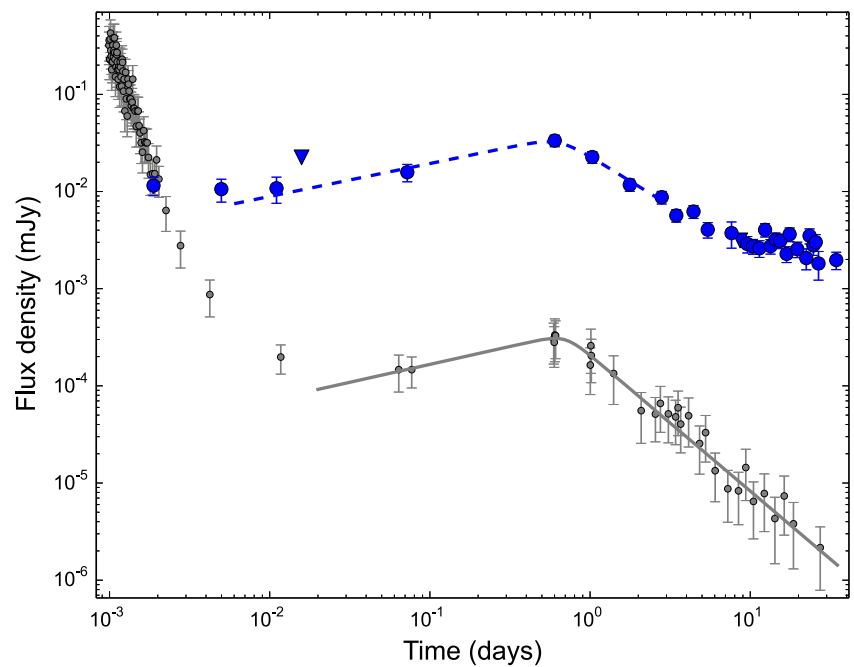

Figure 7. Broken power-law fit to the X-ray (gray, solid) and Swift/UVOT White-band light curves for GRB 100418A near the re-brightening around $0.6 \mathrm{~d}$. The X-ray fit includes points between 0.02 and $50 \mathrm{~d}$, while the Whiteband fit includes points between $6 \times 10^{-3}$ and $3.1 \mathrm{~d}$. A joint fit yields the bestfit parameters $t_{\mathrm{b}}=0.58_{-0.34}^{+0.37} \mathrm{~d}, \alpha_{1}=0.42_{-0.13}^{+0.31}$, and $\alpha_{2}=-1.10_{-0.21}^{+0.15}$, with a fixed value of $y=5.0$ (Section 6.1.1).

using the above time-resolved spectra. We also analyze all UVOT photometry for this burst and report our results in Table 6.

The X-ray light curve up to $8 \times 10^{-3} \mathrm{~d}$ declines steeply as $\alpha_{\mathrm{X}}=-4.52 \pm 0.14$, transitioning to a plateau, at about $0.01 \mathrm{~d}$, followed by a re-brightening. The X-ray observations between $0.02 \mathrm{~d}$ and $5.5 \mathrm{~d}$ can be well fit with a broken power-law model, with $t_{\mathrm{b}}=0.54 \pm 0.18 \mathrm{~d}, F_{\nu, \mathrm{X}}\left(t_{\mathrm{b}}\right)=0.32 \pm 0.08 \mu \mathrm{Jy}$, $\alpha_{1}=0.46 \pm 0.20$, and $\alpha_{2}=-1.15 \pm 0.10$, with the smoothness parameter fixed at $y=5.0$. A broken power-law fit to the UVOT White-band data between $6 \times 10^{-3} \mathrm{~d}$ and $3.1 \mathrm{~d}$ yields $t_{\mathrm{b}}=0.64 \pm 0.17 \mathrm{~d}, \quad F_{\nu, \text { White }}\left(t_{\mathrm{b}}\right)=32 \pm 4 \mu \mathrm{Jy}$, $\alpha_{1}=0.34 \pm 0.09$, and $\alpha_{2}=-1.02 \pm 0.18$, with $y=5.0$ (Figure 7). The decline rate in the White band following the rebrightening is consistent with the decline rate in the $R$-band ${ }^{15}$ between $0.4 \mathrm{~d}$ and $10 \mathrm{~d}\left(\alpha_{\mathrm{R}}=-0.97 \pm 0.08\right)$ and in the $H$-band between the PAIRITEL observations at $0.46 \mathrm{~d}$ and $1.49 \mathrm{~d}\left(\alpha_{\mathrm{H}}=-1.0 \pm 0.1\right)$. Thus, the break time and rise and decay rates of the re-brightening in the X-ray and optical bands are consistent. Finally, we fit the X-ray and UVOT White-band data jointly, where we constrain the model light curves in the two bands to have the same rise and decay rate and time of peak with independent normalizations. Using a Markov Chain Monte Carlo simulation using EMCEE (Foreman-Mackey et al. 2013), we find $t_{\mathrm{b}}=0.58_{-0.34}^{+0.37} \mathrm{~d}, \alpha_{1}=0.42_{-0.13}^{+0.31}$, and $\alpha_{2}=-1.10_{-0.21}^{+0.15}$, with a fixed value of $y=5.0$.

We extract XRT spectra at $0.06-0.08 \mathrm{~d}$ (mean photon arrival $0.07 \mathrm{~d}$ ), and at $0.58-1.81 \mathrm{~d}$ (mean photon arrival $0.88 \mathrm{~d}$ ). We extrapolate the second SED to $1.5 \mathrm{~d}$ and show the complete optical to X-ray SED at $0.07 \mathrm{~d}$ and $1.5 \mathrm{~d}$ in Figure 8 . The spectral index between the UVOT $B$-band observation at $0.07 \mathrm{~d}$ and the X-rays is $\beta_{\mathrm{opt}-\mathrm{X}}=-0.93 \pm 0.09$, which is consistent

\footnotetext{
15 The BYU/WMO $R$-band detection at $35.25 \mathrm{~d}$ reported by Moody et al (2010) is about $0.5 \mathrm{mag}$ brighter than observations from KPNO/SARA (Updike et al. 2010a) and VLT/X-shooter Malesani \& Palazzi (2010) at a similar time, suggesting that the BYU data point may be plagued by a typo. We do not include the BYU/WMO R-band data point at $35.25 \mathrm{~d}$ in our analysis.
} 


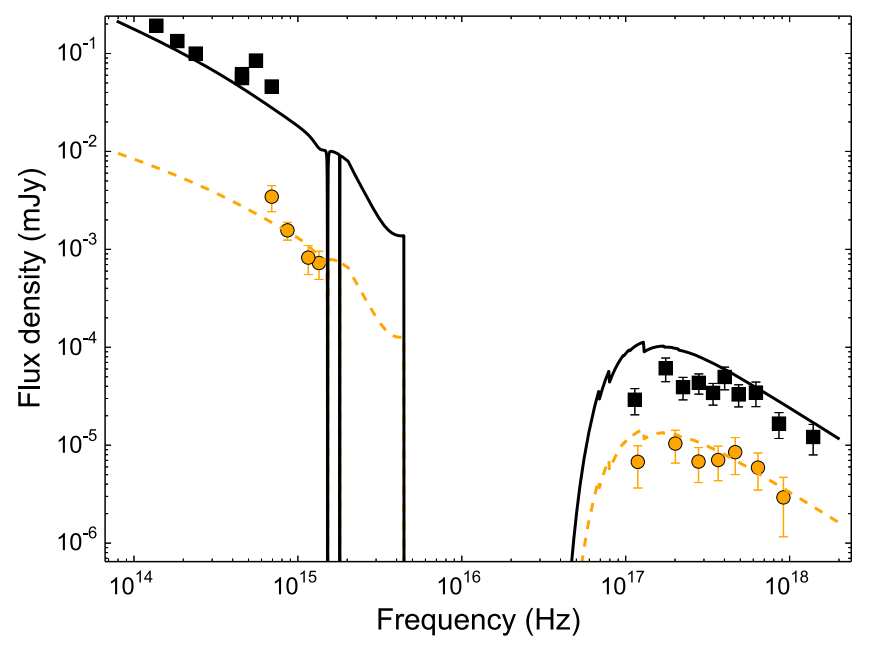

Figure 8. Observed NIR to X-ray SED of the afterglow of GRB 100418A at $0.07 \mathrm{~d}$ (orange circles) and after the re-brightening ( $1.5 \mathrm{~d}$; black squares), together with the best-fit ISM model (Section 6.1.2) including energy injection (Section 6.1.3) at $0.07 \mathrm{~d}$ (orange; dashed) and $1.5 \mathrm{~d}$ (black; solid). The data points and model at $0.07 \mathrm{~d}$ have been multiplied by a factor of 0.1 for clarity. The XRT SED at $1.5 \mathrm{~d}$ has been extrapolated from $0.88 \mathrm{~d}$ using the best-fit broken power-law model to the XRT light curve (Figure 7; the correction factor is $\approx 0.5$ ). The optical data have been extrapolated using the best fit to the Swift/ UVOT light curve (the corrections are small at $<5 \%$ ). The cooling break (visible in the orange, dashed curve at $\approx 3 \times 10^{14} \mathrm{~Hz}$ ) is already below the optical bands at 0.07; the NIR to X-ray frequencies are therefore on the same part of the afterglow SED at $1.5 \mathrm{~d}$.

with the X-ray spectral index of $\beta_{X}=-1.08 \pm 0.18$ over this period, suggesting that the optical and $\mathrm{X}$-ray are located on the same part of the afterglow SED at $0.07 \mathrm{~d}$. The spectral index between the NIR and X-rays at $1.5 \mathrm{~d}$ is $\beta_{\mathrm{NIR}-\mathrm{X}}=-0.94 \pm 0.04$, which is consistent with the spectral index in the NIR and optical alone, $\beta_{\mathrm{NIR}-\mathrm{opt}}=-0.8 \pm 0.1$, once again suggesting that the NIR/optical and X-rays are located on the same part of the afterglow SED at $1.5 \mathrm{~d}$ and that intrinsic extinction is negligible. This implies that either $\nu_{\mathrm{m}}<\nu_{\mathrm{NIR}}<\nu_{\mathrm{X}}<\nu_{\mathrm{c}}$ with $p \approx 3$ or $\nu_{\mathrm{m}}, \nu_{\mathrm{c}}<\nu_{\mathrm{NIR}}<\nu_{\mathrm{X}}$ with $p \approx 2$ at both $0.07 \mathrm{~d}$ and $1.5 \mathrm{~d}$. If $\nu_{\mathrm{m}}<\nu_{\mathrm{NIR}}<\nu_{\mathrm{X}}<\nu_{\mathrm{c}}$ at $1.5 \mathrm{~d}$, the decay rate would be $\alpha \approx-1.5$ in the ISM model or $\alpha \approx-2.0$ in the wind model, whereas in the latter we would expect $\alpha \approx-1$ in both ISM and wind scenarios. The observed $\mathrm{UV} / \mathrm{NIR} / \mathrm{X}$-ray common decay rate after the re-brightening of $\alpha \approx-1$ indicates that $\nu_{\mathrm{m}}, \nu_{\mathrm{c}}<\nu_{\mathrm{NIR}}<\nu_{\mathrm{X}}$ at $1.5 \mathrm{~d}$ and $p \approx 2$.

The $8.46 \mathrm{GHz}$ light curve drops as $t^{-2.5}$ between the ATCA observation at $2 \mathrm{~d}$ and the VLA observation at $3 \mathrm{~d}$, while the SMA $345 \mathrm{GHz}$ light curve is flat over this period (Figure 9). At $\approx 2 \mathrm{~d}$, the radio spectral index is $\approx 0.7$ from 4.9 to $8.46 \mathrm{GHz}$, and $\approx 0.3$ from 8.46 to $345 \mathrm{GHz}$. The rapid decline in the light curve at a single waveband can not be explained in the standard synchrotron model by forward shock emission. We consider an alternative scenario, in which the radio to millimeter emission at $2 \mathrm{~d}$ is dominated by a RS. In that case, the peak frequency of the RS must pass through $8.46 \mathrm{GHz}$ before $2 \mathrm{~d}$ with a peak flux density of at least $\approx 1.3 \mathrm{mJy}$. Propagating the RS spectrum back in time assuming the peak frequency and peak flux evolve as $t^{-1.5}$ and $t^{-1}$, respectively (Kobayashi \& Sari 2000), we would expect a minimum $R$-band flux density of $\approx 2 \mathrm{Jy}$ at $120 \mathrm{~s}$. This is more than five orders of magnitude brighter than Swift White-band observations at this time. Thus the rapid decline and re-brightening of the $8.46 \mathrm{GHz}$ light curve between 2 and $20 \mathrm{~d}$ cannot be explained in the standard synchrotron framework. It is possible that these data suffer from strong ISS, and we therefore exclude these data from our analysis.

\subsubsection{Forward Shock Model at $t \gtrsim 0.4 d$}

The optical and X-ray light curves after $0.4 \mathrm{~d}$ are insensitive to the circumburst density profile since $\nu_{\mathrm{m}}, \nu_{\mathrm{c}}<\nu_{\mathrm{NIR}}<\nu_{\mathrm{X}}$ after the re-brightening. The best-sampled radio light curve is the composite $8.46 \mathrm{GHz}$ formed from VLA, VLBA, and ATCA observations. However, this light curve exhibits significant scatter about a smooth power-law evolution, possibly due to either ISS or inter-calibration issues across the three telescopes. ${ }^{16}$ Thus we are unable to constrain the circumburst density profile from the afterglow data. For the remainder of this section we focus on the ISM model, discussing the possibility of a wind environment in Appendix C.

Since this event occurred at a relatively low redshift, $z=0.6235$ (Antonelli et al. 2010), the host galaxy is detected in the Sloane Digital Sky Survey (SDSS) as SDSS J170527.10 +112742.5 . We use the SDSS photometry $(u=24.55 \pm 1.14$, $g=22.92 \pm 0.17, \quad r=22.45 \pm 0.17, \quad i=21.95 \pm 0.17$, $z=22.54 \pm 0.83$ ) as fixed a priori measurements of the host galaxy flux in the griz bands. We compute the host flux density at $B$ - and $V$-band using $B=g+0.33(g-r)+0.2$ and $V=g-0.58(g-r)-0.01$ (Jester et al. 2005) and hold these values fixed for our MCMC analysis. Since there is no a priori measurement of the host flux density in the UVOT/ White-band and much of the UVOT data was taken in Whiteband, we keep the host flux density in the White-band as a free parameter, and integrate over the GRB SED using the Whiteband filter function. ${ }^{17}$

Using our MCMC analysis as described in Section 4, we fit the data after $0.4 \mathrm{~d}$, and our highest-likelihood model (Figure 9) has the parameters $p \approx 2.1, \epsilon_{\mathrm{e}} \approx 0.12, \epsilon_{B} \approx 1.1 \times 10^{-2}, n_{0} \approx$ $1.4 \mathrm{~cm}^{-3}, E_{\mathrm{K} \text {,iso }} \approx 3.4 \times 10^{52} \mathrm{erg}, t_{\text {jet }} \approx 17 \mathrm{~d}, A_{\mathrm{V}} \lesssim 0.1 \mathrm{mag}$,

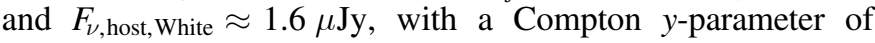
$\approx 2.9$, indicating IC cooling is important. The blastwave Lorentz factor is $\Gamma=4.9(t / 1 \mathrm{~d})^{-3 / 8}$ and the jet opening angle is $\theta_{\text {jet }} \approx 20^{\circ}$. The beaming-corrected kinetic energy is $E_{\mathrm{K}} \approx 2.1 \times 10^{51} \mathrm{erg}$, while the beaming corrected $\gamma$-ray energy is $E_{\gamma} \approx 6 \times 10^{49} \mathrm{erg}\left(1-10^{4} \mathrm{keV}\right.$; rest frame), corresponding to an extremely low radiative efficiency of $\eta_{\mathrm{rad}} \equiv E_{\gamma} /\left(E_{\mathrm{K}}+E_{\gamma}\right) \approx 3 \%$. These results are summarized in Table 5. We present histograms of the marginalized posterior density for all parameters in Figure 10 and contour plots of correlations between the physical parameters in Figure 11.

In concordance with the basic analysis outlined above, we find that the break frequencies at $1 \mathrm{~d}$ for this model are located at $\nu_{\mathrm{a}} \approx 9.0 \mathrm{GHz}, \nu_{\mathrm{m}} \approx 3.4 \times 10^{11} \mathrm{~Hz}$, and $\nu_{\mathrm{c}} \approx 2.3 \times$ $10^{14} \mathrm{~Hz}$, while the peak flux density is about $12 \mathrm{mJy}$ at $\nu_{\mathrm{m}}$. The spectrum transitions from fast to slow cooling at about $230 \mathrm{~s}$ after the burst, and $\nu_{\mathrm{m}}$ drops below $\nu_{\mathrm{a}}$ at about $25 \mathrm{~d}$. The high value of $\nu_{\mathrm{a}}$ is required to suppress the flux density at $8.46 \mathrm{GHz}$ and $5 \mathrm{GHz}$ (VLA), relative to the mm-band (PdBI and SMA). The ordering of the break frequencies relative to the observing bands, $\nu_{\mathrm{m}}, \nu_{\mathrm{c}}<\nu_{\mathrm{NIR}}$ at $1 \mathrm{~d}$, ensures that the optical and X-ray are on the same part of the synchrotron spectrum at this time. The jet break at $\approx 17 \mathrm{~d}$ is largely driven by the millimeter

\footnotetext{
16 To avoid the radio data from driving the fit, we apply an uncertainty floor of $15 \%$ to the $\mathrm{cm}$ - and mm-band observations.

17 http://www.swift.ac.uk/analysis/uvot/filters.php
} 

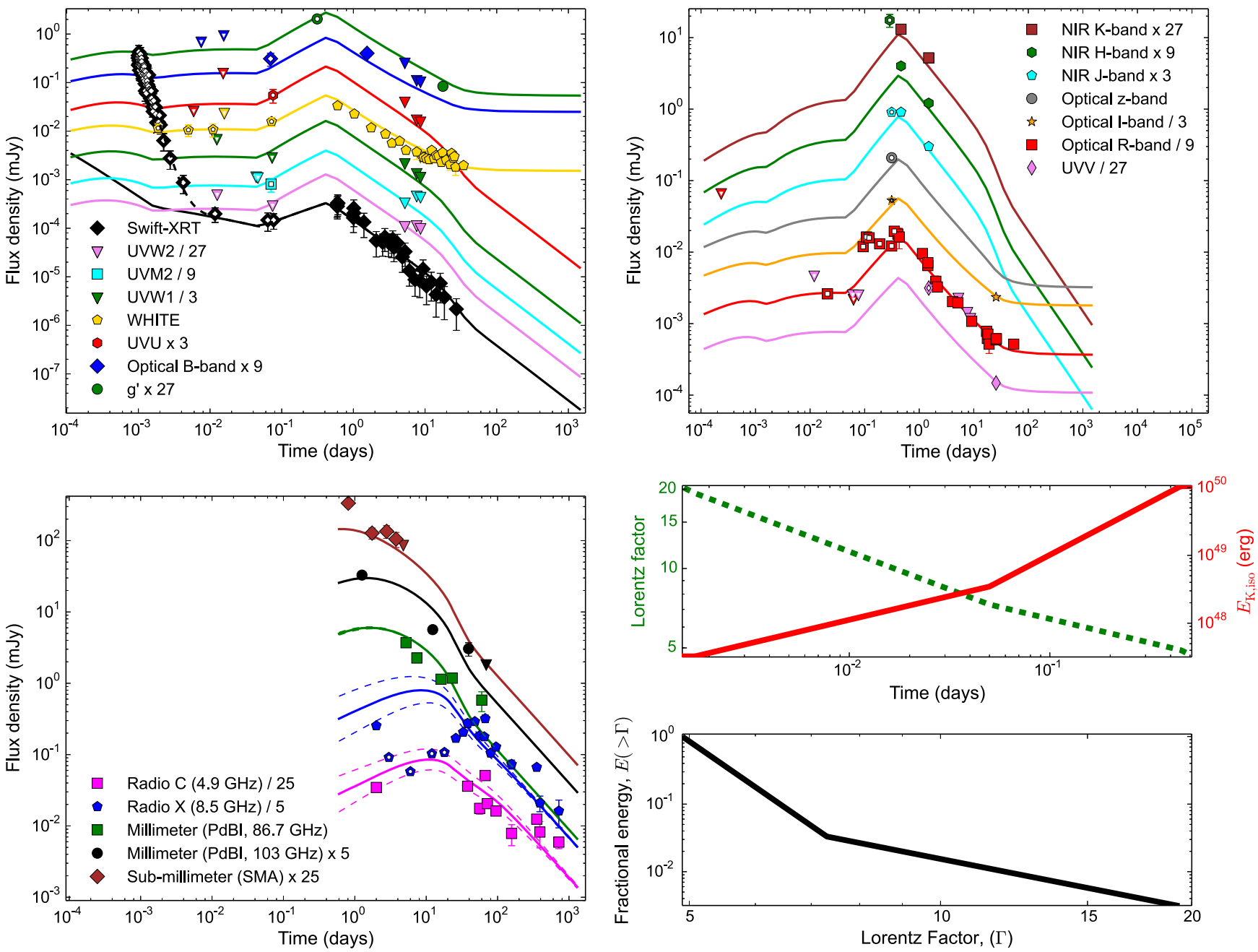

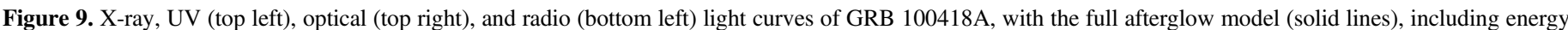

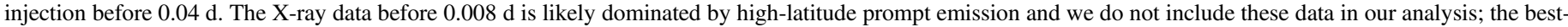

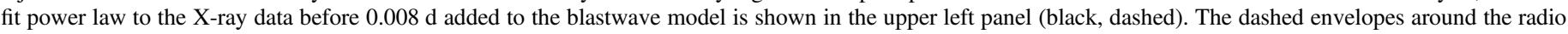

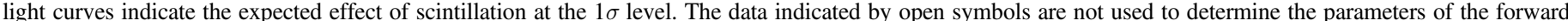

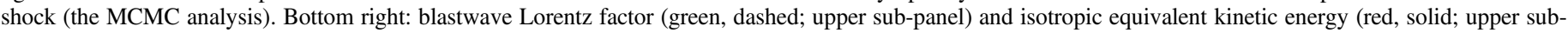

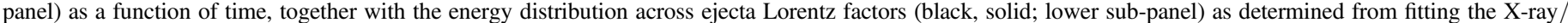
$\mathrm{UV} /$ optical re-brightening at $0.7 \mathrm{~d}$.

and radio data. The blastwave becomes non-relativistic at $t_{\mathrm{NR}} \approx$ $40 \mathrm{~d}$, resulting in a subsequent slow decline $\left(F_{\nu} \sim t^{0.6+3 \beta} \sim t^{-1}\right.$, with $\beta=(1-p) / 2 \approx-0.5$; Frail et al. 2000) in the radio light curves after the peak.

Moin et al. (2013) use a VLBA limit on the size of the afterglow at $65 \mathrm{~d}$ to estimate the average ejecta Lorentz factor. However, we note that the apparent physical size of the afterglow is given by $R_{\perp}=\left[2^{12-3 k}(4-k)^{5-k} /(5-k)^{5-k}\right]^{1 /(8-2 k)}$ $\Gamma c t_{\mathrm{z}} \approx 2.3 \Gamma c t_{\mathrm{z}}$, where $\gamma_{l}$ is the ejecta Lorentz factor of the ejecta at time, $t_{\mathrm{z}}=t_{\mathrm{obs}} /(1+z)$, and $k=0$ for the ISM model (Granot \& Sari 2002); therefore the VLBI measurement actually represents an upper limit to the ejecta Lorentz factor at the time of the measurement, such that $\Gamma\left(t_{\mathrm{obs}}=65 \mathrm{~d}\right)<5.8$. Since the blastwave becomes non-relativistic at around 40 days, our model does not violate the VLBA limit on the angular size of the afterglow.

\subsubsection{Energy Injection Model}

Taking the forward shock model described in Section 6.1.2 as a starting point, we find that the X-ray and UV/optical data before the re-brightening can be explained by two successive periods of energy injection,

$$
\begin{aligned}
& E_{\mathrm{K}, \text { iso }}(t) \\
& = \begin{cases}E_{\mathrm{K}, \text { iso }, \mathrm{f}}, & t>t_{0}=0.45 \mathrm{~d} \\
E_{\mathrm{K}, \text { iso }, \mathrm{f}}\left(\frac{t}{t_{0}}\right)^{1.55}, & t_{1}=0.05 \mathrm{~d}<t<t_{0} \\
E_{\mathrm{K}, \text { iso }, \mathrm{f}}\left(\frac{t_{1}}{t_{0}}\right)^{1.55}\left(\frac{t}{t_{1}}\right)^{0.7}, & t_{2}=1.7 \times 10^{-3} \mathrm{~d}<t<t_{1} \\
E_{\mathrm{K}, \text { iso }, \mathrm{f}}\left(\frac{t_{1}}{t_{0}}\right)^{1.55}\left(\frac{t_{2}}{t_{1}}\right)^{0.7}, & t<t_{2} .\end{cases}
\end{aligned}
$$

In this model, the energy increases by a factor of $\approx 11$ from $E_{\mathrm{K} \text {,iso,i }} \approx 1.0 \times 10^{50} \mathrm{erg}$ at $1.7 \times 10^{-3} \mathrm{~d}$ to $E_{\mathrm{K} \text {,iso }} \approx$ $1.1 \times 10^{51} \mathrm{erg}$ at $0.05 \mathrm{~d}$ followed by another increase by a factor of $\approx 30$ to $E_{\mathrm{K} \text {,iso,f }} \approx 3.4 \times 10^{52} \mathrm{erg}$ at $0.45 \mathrm{~d}$. The overall 

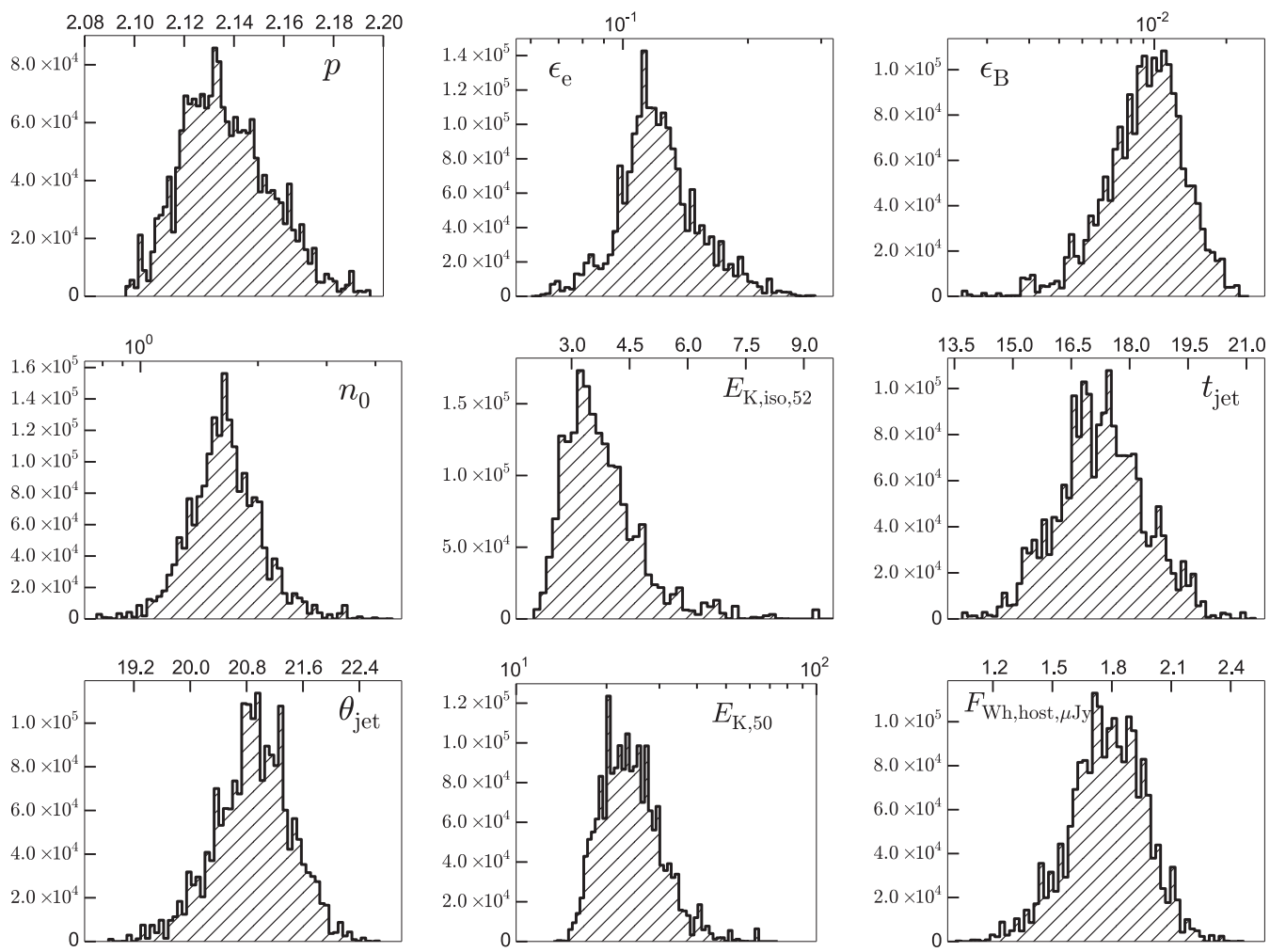

Figure 10. Posterior probability density functions for the physical parameters for GRB 100418A in a constant density environment from MCMC simulations.
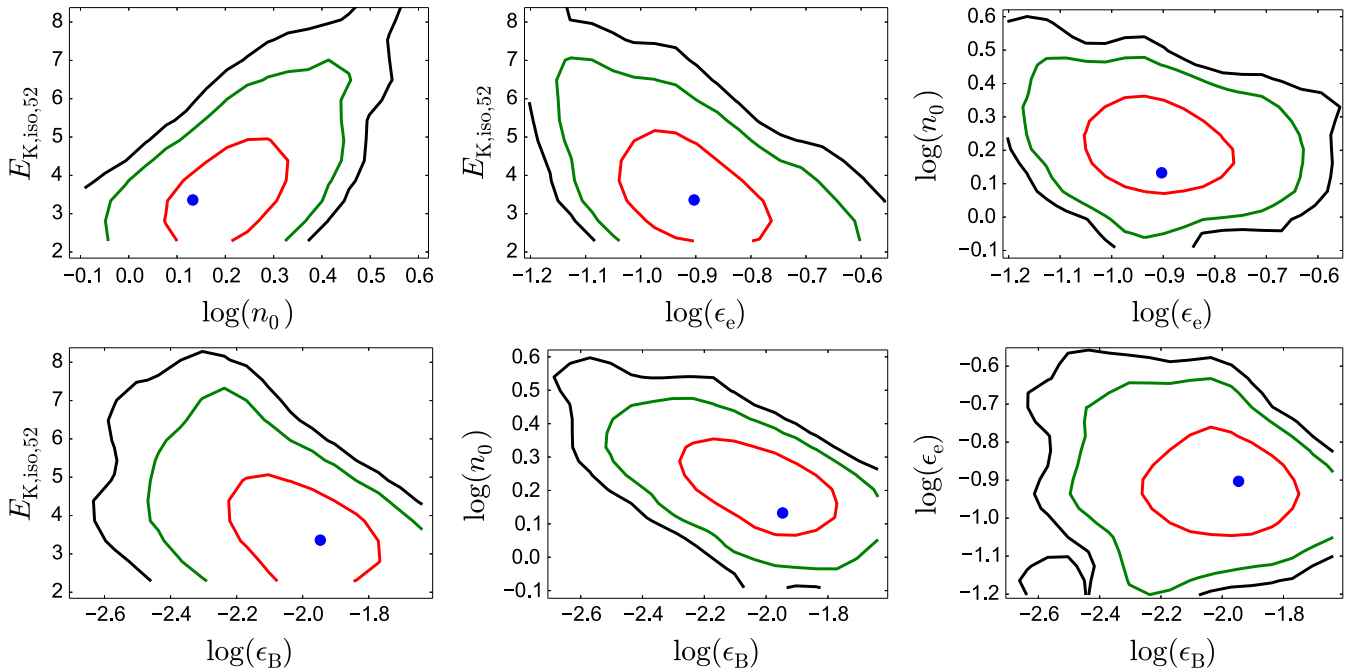

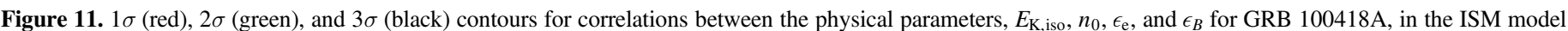

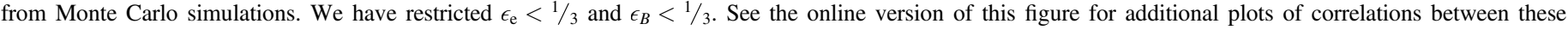

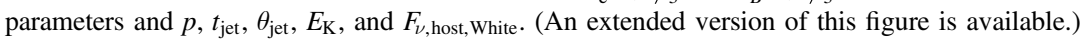

increase in energy is a factor of $\approx 340$, corresponding to an injected energy fraction of $\approx 99.7 \%$ over this period. In comparison, $E_{\gamma, \text { iso }}=9.9_{-3.4}^{+6.3} \times 10^{50}$ (Marshall et al. 2011). The blastwave Lorentz factor decreases from $\Gamma \approx 38$ at the start of energy injection at $1.7 \times 10^{-3}$ to $\Gamma \approx 11$ at $0.05 \mathrm{~d}$, and then to $\Gamma \approx 7$ at the end of energy injection at $0.45 \mathrm{~d}$. The values of $m$ derived above correspond to an ejecta distribution with $E(>\Gamma) \propto \Gamma^{1-s}$ with $s \approx 9.6$ for $7 \lesssim \Gamma \lesssim 11$ and $s \approx 3$ for $11 \lesssim \Gamma \lesssim 38$.

\subsection{GRB 100901A}

\subsubsection{GRB Properties and Basic Considerations}

GRB 100901A was detected and localized by the Swift BAT on 2010 September 01 at 13:34:10 UT (Immler et al. 2010). The burst duration is $T_{90}=(439 \pm 33) \mathrm{s}$, with a fluence of $F_{\gamma}=(2.1 \pm 0.3) \times 10^{-6} \mathrm{erg} \mathrm{cm}^{-2} \quad(15-150 \mathrm{keV}$ observer frame; Immler et al. 2010). The afterglow was detected in the X-rays and optical bands by XRT (Page \& Immler 2010), 
Table 7

Swift/UVOT Observations of GRB 100901A

\begin{tabular}{lccccc}
\hline \hline $\begin{array}{l}t-t_{0} \\
\text { (days) }\end{array}$ & Filter & $\begin{array}{c}\text { Frequency } \\
(\mathrm{Hz})\end{array}$ & $\begin{array}{c}\text { Flux } \\
\text { Density } \\
(\mu \mathrm{Jy})\end{array}$ & $\begin{array}{c}\text { Uncertainty } \\
(\mu \mathrm{Jy})\end{array}$ & $\begin{array}{c}\text { Detection? } \\
(1=\text { Yes })\end{array}$ \\
\hline 0.00258 & White & $8.64 \mathrm{e}+14$ & 27.4 & 5.65 & 1 \\
0.00498 & $u$ & $8.56 \mathrm{e}+14$ & 146 & 11.4 & 1 \\
0.00661 & $b$ & $6.92 \mathrm{e}+14$ & 230 & 75.6 & 0 \\
0.00689 & White & $8.64 \mathrm{e}+14$ & 58.6 & 18.9 & 1 \\
0.00717 & $u v w 2$ & $1.48 \mathrm{e}+15$ & 67.9 & 12.1 & 0
\end{tabular}

Note.

a In cases of non-detections, we report the formal flux measurement at the position of the afterglow.

(This table is available in its entirety in machine-readable form.)

UVOT (Pritchard \& Immler 2010), and by multiple groundbased observatories (e.g., de Cia et al. 2010; Hentunen et al. 2010; Gorbovskoy et al. 2010; Kopac et al. 2010; Kuroda et al. 2010c; Pandey \& Zheng 2010; Sahu et al. 2010b; Updike et al. 2010b), as well as in the radio by the WSRT and the VLA (Chandra \& Frail 2010a; van der Horst et al. 2010b). Spectroscopic observations with Magellan yielded a redshift of $z=1.408$ (Chornock et al. 2010). Using the BAT fluence, Gorbovskoy et al. (2012) determined the isotropic equivalent $\gamma$-ray energy of this burst to be $E_{\gamma \text {,iso }}=6.3 \times 10^{52} \mathrm{erg}$ (1-10 $0^{4} \mathrm{keV}$; rest frame). However, the $\gamma$-ray spectrum does not exhibit a turnover within the BAT energy range, indicating that the peak of the $\gamma$-ray spectrum, $E_{\text {peak }}$, is outside the BAT energy range. Margutti et al. (2013) obtain a correlation between $E_{\mathrm{BAT}, \mathrm{obs}}(15-150 \mathrm{keV})$ and $E_{\gamma, \text { iso }}$ : $\frac{E_{\gamma, \text { iso }}}{\text { erg }}=10^{-3.7}\left[\frac{E_{\mathrm{BAT}}^{15-150}}{\mathrm{erg}}\right]^{1.08 \pm 0.01}$ with a scatter of $\sigma=0.24$ $(68 \%)$, which we use to perform a K-correction and obtain $E_{\gamma, \text { iso }}=(8 \pm 1) \times 10^{52} \mathrm{erg}$. Note that the error bars do not include the uncertainty in the correlation itself.

XRT began observing the GRB during the $\gamma$-ray emission interval. The X-ray spectra from $1 \mathrm{ks}$ to the end of XRT observations are well fit by an absorbed single power-law model with $N_{\mathrm{H}, \mathrm{Gal}}=7.1 \times 10^{20} \mathrm{~cm}^{-2}$ (Kalberla et al. 2005), intrinsic hydrogen column $N_{\mathrm{H} \text {,int }}=(3.1 \pm 0.7) \times 10^{21} \mathrm{~cm}^{-2}$, and photon index, $\Gamma_{\mathrm{X}}=2.15 \pm 0.06$, with no evidence for spectral evolution during this period. We convert the count rate light curve published by Margutti et al. (2013) to a flux density light curve at $1 \mathrm{keV}$ using their time-resolved spectra. We also analyze all UVOT photometry for this burst and report our results in Table 7 , and a compilation of all photometry listed in GCN circulars ${ }^{18}$ or published elsewhere for this event in Table 8.

The X-ray data before the first orbital gap at around $10^{-2} \mathrm{~d}$ exhibits rapid flaring, ending in a steep decay. The earliest UVOT observations during this period detect a counterpart at multiple wavelengths. Owing to the contemporaneous $\gamma$-ray emission, indicating on-going central engine activity during this period, we do not consider data before the first orbital gap for our afterglow analysis. The X-ray re-brightening and subsequent

\footnotetext{
18 The KPNO/SARA $V$-band detection (Updike et al. 2010b) is significantly brighter than expected from interpolating the UVOT $V$-band light curve, implying a difference either in calibration or in the filter response between the two instruments. We do not include the KPNO $V$-band data point at $0.78 \mathrm{~d}$ in our analysis.
}

fading between $0.08 \mathrm{~d}$ and $20 \mathrm{~d}$ can be well fit with a broken power-law model (Equation (5)), with $t_{\mathrm{b}}=0.40 \pm$ $0.02 \mathrm{~d}, \quad F_{\nu, \mathrm{X}}\left(t_{\mathrm{b}}\right)=2.0 \pm 0.1 \mu \mathrm{Jy}, \quad \alpha_{1}=0.77 \pm 0.15, \quad \alpha_{2}=$ $-1.55 \pm 0.06$, and $y=2.3 \pm 1.1$ (Figure 12).

A broken power-law fit to the UVOT $U$-band data between $0.02 \mathrm{~d}$ and $20 \mathrm{~d}$ yields $t_{\mathrm{b}}=0.40 \pm 0.03 \mathrm{~d}, F_{\nu, \mathrm{U}}\left(t_{\mathrm{b}}\right)$ $=144 \pm 7 \mu \mathrm{Jy}, \alpha_{1}=0.45 \pm 0.06$, and $\alpha_{2}=-1.63 \pm 0.12$, with $y=2.5$, similar to the results from fitting the X-ray re-brightening. Over the same time range, a broken power-law fit to the UVOT $B$-band data yields $t_{\mathrm{b}}=0.39 \pm 0.08 \mathrm{~d}$, $F_{\nu, \mathrm{B}}\left(t_{\mathrm{b}}\right)=201 \pm 24 \mu \mathrm{Jy}, \quad \alpha_{1}=0.40 \pm 0.06, \quad$ and $\quad \alpha_{2}=$ $-1.46 \pm 0.21$, similar to the X-ray and $U$-band fits. Finally, we fit the X-ray, UVOT $U$-band, and UVOT $B$-band data jointly, where we constrain the model light curves at the two frequencies to have the same rise and decay rate and time of peak, and use independent normalizations in the three bands. Using a Markov Chain Monte Carlo simulation using EMCEE (Foreman-Mackey et al. 2013), we find $t_{\mathrm{b}}=$ $0.36 \pm 0.02, \quad \alpha_{1}=0.57 \pm 0.09, \quad$ and $\quad \alpha_{2}=-1.47 \pm 0.07$, with a fixed value of $y=2.5$.

The spectral index between the UKIRT $K$-band observation at $2.88 \mathrm{~d}$ (Im et al. 2010) and the $\mathrm{X}$-rays is $\beta_{\mathrm{NIR}, \mathrm{x}}=$ $-0.78 \pm 0.08$. The spectral index between the NIR $K$-band and the $S$ wift $/ U$-band is slightly steeper, $\beta_{\mathrm{NIR}, \mathrm{UV}}=-1.0 \pm 0.2$, indicating that some extinction may be present. The spectral slope in the $\mathrm{X}$-rays following the re-brightening is $\beta_{\mathrm{X}}=-1.15 \pm 0.06$, leading to $\beta_{\mathrm{NIR}, \mathrm{X}}-\beta_{\mathrm{X}}=0.4 \pm 0.1$ suggesting that $\nu_{\mathrm{opt}}<\nu_{\mathrm{c}}<\nu_{\mathrm{X}}$ at $2.88 \mathrm{~d}$ (Figure 13).

If the X-rays indeed lie above $\nu_{\mathrm{c}}$, we would have $p=-2 \beta_{\mathrm{X}}=2.3 \pm 0.12$. The expected light curve decay rate is $\alpha=(2-3 p) / 4=-1.2 \pm 0.1$ above $\nu_{\mathrm{c}}$ and $\alpha=$ $3(1-p) / 4=-1.0 \pm 0.1$ (ISM) or $\alpha=-1.5 \pm 0.1$ (wind) below $\nu_{\mathrm{c}}$. The $U$-band light curve after the re-brightening can be fit either with a single power law with $\alpha=-1.5$ or with a break from $\alpha \approx-0.9$ to $\alpha \approx-1.8$ at $\approx 1 \mathrm{~d}$ and $\alpha=-1.0 \pm 0.1$ between $0.2 \mathrm{~d}$ and $1.0 \mathrm{~d}$. The former is consistent with a wind-like environment, while the latter suggests an ISM model and a jet break at $\approx 1 \mathrm{~d}$. We return to this point later.

There are multiple epochs of observations with the VLA (PI: Kulkarni) and with WSRT (PI: van der Horst) in the NRAO and ASTRON archives. We downloaded and analyzed these observations with CASA using techniques described in Section 2.5. We list our derived radio photometry for the afterglow of GRB 100901A in Table 9, and additionally include the SMA observation of the afterglow at $345 \mathrm{GHz}$ reported by de Ugarte Postigo et al. (2007) in our analysis.

Extrapolating the Lightbuckets $R$-band data at $1.7 \mathrm{~d}$ to the time of the SMA $345 \mathrm{GHz}$ upper limit at 1.8 days using the fit to the optical $U$-band light curve described above, we find a spectral index of $\beta_{\mathrm{mm}-\mathrm{R}} \gtrsim-0.6$ from the millimeter to the optical at this time. Combined with the steeper spectrum of $\beta_{\mathrm{NIR}, \mathrm{X}}=-0.78 \pm 0.08$ between the optical and X-rays, this suggests that the spectrum turns over above the millimeter band, indicating that $\nu_{\mathrm{m}} \gtrsim 345 \mathrm{GHz}$ at $1.8 \mathrm{~d}$.

The spectral index between the VLA $4.5 \mathrm{GHz}$ and $7.9 \mathrm{GHz}$ bands at $4.92 \mathrm{~d}$ is $\beta=0.9 \pm 0.3$. However, the light curve at $4.5 \mathrm{GHz}$ declines as $\alpha=-0.17 \pm 0.12$ between $3 \mathrm{~d}$ and $12 \mathrm{~d}$, and as $\alpha=-1.1 \pm 0.5$ between $12 \mathrm{~d}$ and $20 \mathrm{~d}$. The rising radio spectrum coupled with the declining light curve implies that the jet break has occurred before $5 \mathrm{~d}$. The steepening of the $4.5 \mathrm{GHz}$ light curve at $\approx 12 \mathrm{~d}$ implies $\nu_{\mathrm{m}} \approx 4.5 \mathrm{GHz}$ at this 
Table 8

Optical Observations of GRB 100901A

\begin{tabular}{|c|c|c|c|c|c|c|c|c|}
\hline $\begin{array}{l}t-t_{0} \\
\text { (days) }\end{array}$ & Observatory & $\begin{array}{l}\text { Telescope/ } \\
\text { Instrument }\end{array}$ & Filter & $\begin{array}{c}\text { Frequency } \\
(\mathrm{Hz})\end{array}$ & $\begin{array}{l}\text { Flux Density }^{\mathrm{a}} \\
\qquad(\mu \mathrm{Jy})\end{array}$ & $\begin{array}{c}\text { Uncertainty } \\
(\mu \mathrm{Jy})\end{array}$ & $\begin{array}{l}\text { Detection? } \\
(1=\text { Yes })\end{array}$ & Reference \\
\hline 0.00131 & MASTER & $\cdots$ & $C R$ & $4.4 \mathrm{e}+14$ & 82.1 & 436 & 0 & Gorbovskoy et al. (2012) \\
\hline 0.00178 & MASTER & $\ldots$ & $C R$ & $4.4 \mathrm{e}+14$ & 156 & 170 & 0 & Gorbovskoy et al. (2012) \\
\hline 0.00246 & MASTER & $\ldots$ & $C R$ & $4.4 \mathrm{e}+14$ & 131 & 143 & 0 & Gorbovskoy et al. (2012) \\
\hline 0.00396 & MASTER & $\ldots$ & $C R$ & $4.4 \mathrm{e}+14$ & 119 & 107 & 0 & Gorbovskoy et al. (2012) \\
\hline 0.00494 & MASTER & $\ldots$ & $C R$ & $4.4 \mathrm{e}+14$ & 304 & 78.6 & 1 & Gorbovskoy et al. (2012) \\
\hline
\end{tabular}

Note.

${ }^{a}$ In cases of non-detections, we report the formal flux measurement at the position of the afterglow.

(This table is available in its entirety in machine-readable form.)

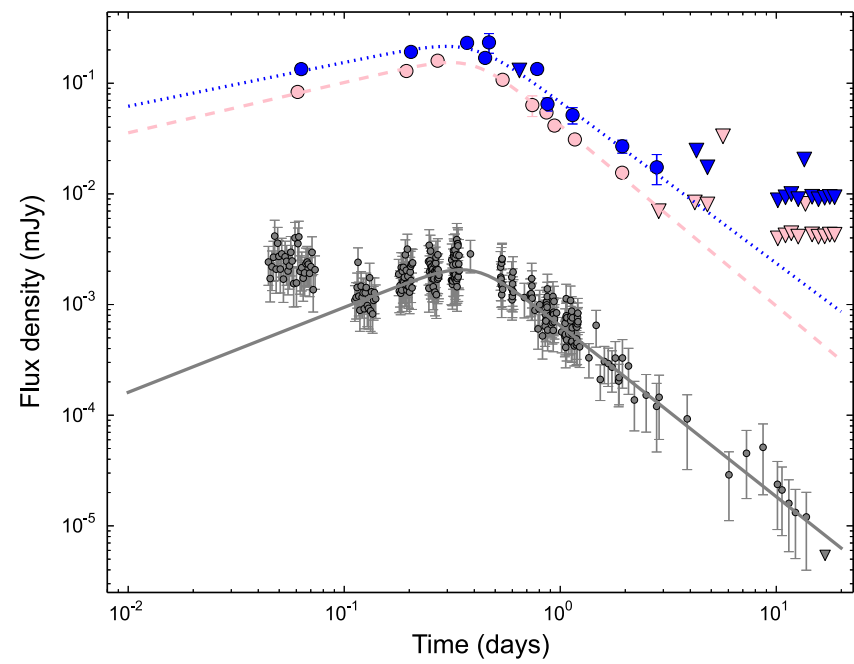

Figure 12. Broken power-law fit to the X-ray (gray, solid), Swift/UVOT $U$-band (pink, dashed), and Swift/UVOT B-band (blue, dotted) light curves for GRB 100901A near the re-brightening around 0.6 d. The X-ray fit includes points between 0.08 and $20 \mathrm{~d}$, while the $U$ - and $B$-band fits include points between $0.02 \mathrm{~d}$ and $20 \mathrm{~d}$. A joint fit yields the best-fit parameters $t_{\mathrm{b}}=0.36 \pm 0.02, \alpha_{1}=0.57 \pm 0.09$, and $\alpha_{2}=-1.47 \pm 0.07$ (Section 6.2.1).

time. Given that $\nu_{\mathrm{m}}>345 \mathrm{GHz}$ at $1.8 \mathrm{~d}$, this would imply an evolution of $\sim \nu_{\mathrm{m}} \propto t^{-2.3}$, consistent with the expected evolution of $\nu_{\mathrm{m}} \propto t^{-2}$ following the jet break. Together, this implies that the jet break must have occurred before $1.8 \mathrm{~d}$, which agrees with the results from the X-ray and optical analysis above and indicates an ISM-like environment. We therefore focus on the ISM model for the remainder of this section, and discuss the wind model for completeness in Appendix D.

To summarize, the NIR to X-ray SED exhibits mild evidence for extinction and suggests that $\nu_{\mathrm{NIR}}<\nu_{\mathrm{c}}<\nu_{\mathrm{X}}$ at $\approx 3 \mathrm{~d}$, with $p=2.3 \pm 0.1$. The radio observations indicate $\nu_{\mathrm{m}} \approx 5 \mathrm{GHz}$ at $\approx 12 \mathrm{~d}$. Together, the $\mathrm{X}$-ray, NIR and radio data suggest $t_{\text {jet }} \approx 1 \mathrm{~d}$ and an ISM-like environment. All radio observations took place after this time, and are therefore insensitive to the density profile of the circumburst environment.

\subsubsection{Forward Shock Model at $t \gtrsim 0.25 d$}

We employ our MCMC analysis to fit the multi-band data for GRB $100901 \mathrm{~A}$ after $t \approx 0.25 \mathrm{~d}$. At the redshift of $z=1.408$, the UVOT White-, $u v w 1-, u v w 2-$, and $u v m 2$-band

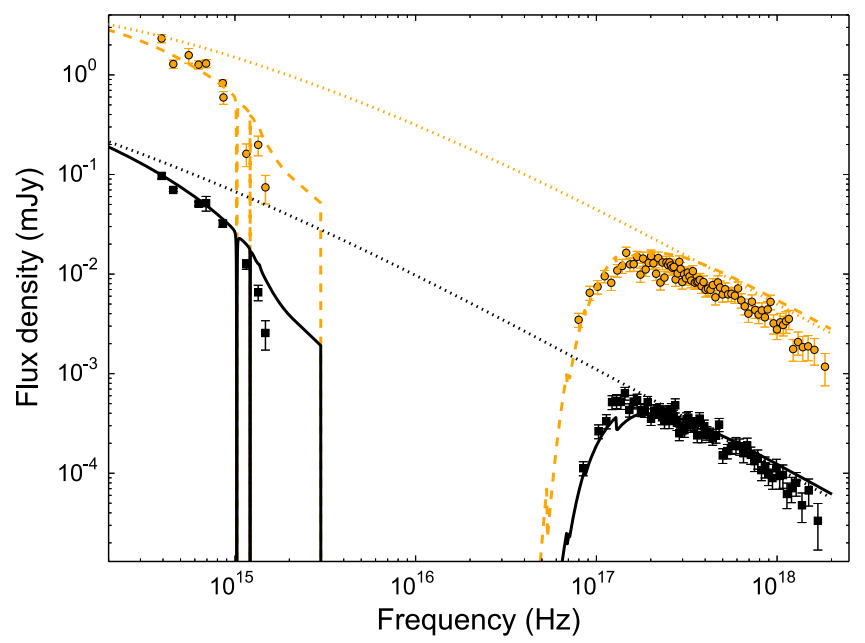

Figure 13. Observed NIR to X-ray SED of the afterglow of GRB 100901A at $0.06 \mathrm{~d}$ (orange circles) and after the re-brightening (1.14 d; black squares), together with the best-fit ISM model (Section 6.2.2) including energy injection (Section 6.2.3) at $0.06 \mathrm{~d}$ (orange; dashed) and $1.14 \mathrm{~d}$ (black; solid). The data points and model at $0.06 \mathrm{~d}$ have been multiplied by a factor of 10 . for clarity. The XRT SEDs at $1.14 \mathrm{~d}$ has been extrapolated from $3 \mathrm{~d}$ using the best-fit broken power-law model to the XRT light curve (Figure 12; the correction factor is $\approx 4.5$ ). The optical data have been extrapolated using the joint best fit to the Swift/XRT and UVOT light curves (the corrections are small at $\lesssim 5 \%$ ). The dotted curves indicate afterglow models with no extinction or IGM absorption. Note that the Swift/UVOT $u v w 1-, u v w 2-$, and $u v m 2$-band data lie blueward of Ly $\alpha$ in the rest frame of the host galaxy $\left(1.0 \times 10^{15} \mathrm{~Hz}\right.$ in the observer frame), and are likely subject to significant IGM absorption. For this reason, we do not include these bands (or the Swift/UVOT White-band, not shown here) to constrain our afterglow model.

data are affected by IGM absorption, and we do not include these bands in our analysis. The highest-likelihood ISM model (Figure 14) the parameters $p \approx 2.03, \epsilon_{\mathrm{e}} \approx 0.33, \epsilon_{B} \approx 0.32$, $n_{0} \approx 3.2 \times 10^{-3} \mathrm{~cm}^{-3}, E_{\mathrm{K}, \text { iso }} \approx 3.0 \times 10^{53} \mathrm{erg}, t_{\mathrm{jet}} \approx 0.96 \mathrm{~d}$, $A_{\mathrm{V}} \approx 0.09 \mathrm{mag}$, and $F_{\nu, \text { host, } \mathrm{r}^{\prime}} \approx 4.1 \mu \mathrm{Jy}$, with a Compton $y$-parameter of $\approx 0.6$. The blastwave Lorentz factor is $\Gamma=15.9(t / 1 \mathrm{~d})^{-3 / 8}$ and the jet opening angle is $\theta_{\text {jet }} \approx 2 \circ 1$. The beaming-corrected kinetic energy is $E_{\mathrm{K}} \approx 2.1 \times 10^{50} \mathrm{erg}$, while the beaming corrected $\gamma$-ray energy is $E_{\gamma} \approx 5 \times 10^{49} \mathrm{erg}$ $\left(1-10^{4} \mathrm{keV}\right.$; rest frame). We plot histograms of the measured parameters in Figure 15 and correlation contours between the physical parameters in Figure 16, providing summary statistics from our MCMC analysis in Table 5.

In this model, the break frequencies at $1 \mathrm{~d}$ are located at $\nu_{\mathrm{a}} \approx 0.7 \mathrm{GHz}, \quad \nu_{\mathrm{m}} \approx 2 \times 10^{12} \mathrm{~Hz}$, and $\nu_{\mathrm{c}} \approx 9 \times 10^{14} \mathrm{~Hz}$, while the peak flux density is about $4.2 \mathrm{mJy}$ at $\nu_{\mathrm{m}}$. The 
Table 9

Millimeter and Radio Observations of GRB 100901A

\begin{tabular}{|c|c|c|c|c|c|c|c|}
\hline $\begin{array}{l}\text { Date } \\
\text { (UT) }\end{array}$ & $\begin{array}{l}t-t_{0} \\
\text { (days) }\end{array}$ & Observatory & $\begin{array}{l}\text { Frequency } \\
\quad(\mathrm{GHz})\end{array}$ & $\begin{array}{l}\text { Integration time } \\
\qquad(\min )\end{array}$ & $\begin{array}{l}\text { Flux density } \\
\qquad(\mathrm{mJy})\end{array}$ & $\begin{array}{l}\text { Uncertainty } \\
\text { (mJy) }\end{array}$ & $\begin{array}{r}\text { Detection? } \\
(1=\text { Yes })\end{array}$ \\
\hline 2010 Sep 06.49 & 4.92 & VLA & 4.495 & 31.3 & 289 & 43 & 1 \\
\hline 2010 Sep 06.49 & 4.92 & VLA & 7.915 & 25.7 & 488 & 37 & 1 \\
\hline 2010 Sep 08.42 & 6.85 & VLA & 4.495 & 26.7 & 247 & 40 & 1 \\
\hline 2010 Sep 08.42 & 6.85 & VLA & 7.915 & 24.3 & 349 & 54 & 1 \\
\hline 2010 Sep 13.46 & 11.90 & VLA & 4.495 & 33.4 & 245 & 38 & 1 \\
\hline 2010 Sep 13.46 & 11.90 & VLA & 7.915 & 28.2 & 419 & 46 & 1 \\
\hline 2010 Sep 13.46 & 11.90 & VLA & 33.559 & 22.3 & 270 & 90 & 0 \\
\hline 2010 Sep 21.44 & 19.87 & VLA & 4.495 & 32.7 & 138 & 32 & 1 \\
\hline 2010 Sep 21.44 & 19.87 & VLA & 7.915 & 32.7 & 209 & 46 & 1 \\
\hline 2010 Sep 21.44 & 19.79 & VLA & 33.559 & 14.7 & 288 & 96 & 0 \\
\hline 2010 Oct 15.18 & 43.61 & VLA & 4.495 & 19.1 & 126 & 42 & 0 \\
\hline 2010 Oct 15.18 & 43.61 & VLA & 7.915 & 19.4 & 213 & 71 & 0 \\
\hline 2010 Oct 15.18 & 43.86 & VLA & 33.559 & 17.6 & 246 & 82 & 0 \\
\hline 2010 Sep 04.10 & 2.54 & WSRT & 4.90 & 567.6 & 189 & 46 & 1 \\
\hline 2010 Sep 07.10 & 5.53 & WSRT & 4.90 & 531.9 & 275 & 61 & 1 \\
\hline 2010 Sep 12.08 & 10.52 & WSRT & 4.90 & 551.9 & 311 & 47 & 1 \\
\hline 2010 Sep 21.05 & 19.48 & WSRT & 4.90 & 525.7 & 252 & 84 & 0 \\
\hline 2010 Sep 42.00 & 40.44 & WSRT & 4.90 & 550.8 & 123 & 41 & 0 \\
\hline 2010 Sep 03.44 & 1.875 & SMA & 345.0 & $\ldots$ & 2250 & 750 & 0 \\
\hline
\end{tabular}

VLA and WSRT data are from this work. SMA data are from de Ugarte Postigo et al. (2007). Dates in column 1 refer to the mid-point of each observation.

spectrum transitions from fast to slow cooling at about $350 \mathrm{~s}$ after the burst. The cooling frequency is located between the optical and X-rays and the jet break is before $1 \mathrm{~d}$, both of which are expected from the basic analysis outlined above. The low value of $p$ is driven by the shallow measured decline in the $\mathrm{X}$-rays following the jet break, and the resulting spectrum remains consistent with the optical-to-X-ray SED (Figure 13).

\subsubsection{Energy Injection Model}

Taking the forward shock model described in Section 6.2.2 as a starting point, we find that the X-ray and UV/optical data before the re-brightening can be explained by a single period of energy injection between $0.105 \mathrm{~d}$ and $\approx 0.26 \mathrm{~d}$ :

$$
E_{\mathrm{K}, \text { iso }}(t)= \begin{cases}E_{\mathrm{K}, \text { iso }, \mathrm{f}}, & t>t_{0}=0.26 \mathrm{~d} \\ E_{\mathrm{K}, \text { iso }, \mathrm{f}}\left(\frac{t}{t_{0}}\right)^{1.88}, & t_{1}=0.105 \mathrm{~d}<t<t_{0} \\ E_{\mathrm{K}, \text { iso }, \mathrm{f}}\left(\frac{t_{1}}{t_{0}}\right)^{1.88}, & t<t_{1} .\end{cases}
$$

In this model, the energy increases by a factor of $\approx 5.5$ from $E_{\mathrm{K}, \text { iso }, \mathrm{i}} \approx 5.4 \times 10^{52} \mathrm{erg}$ at $0.105 \mathrm{~d}$ to $E_{\mathrm{K}, \text { iso } \mathrm{f}} \approx 3.0 \times 10^{53} \mathrm{erg}$ at $0.26 \mathrm{~d}$, corresponding to an injected energy fraction of $\approx 85 \%$, while the Lorentz factor decreases from $\Gamma \approx 30$ to $\Gamma \approx 26$ over this period. In comparison, $E_{\gamma, \text { iso }} \approx 8 \times 10^{52} \mathrm{erg}$ (Section 6.2.1). The value of $m \approx 1.88$ corresponds to $s \approx 14.4$ for $26 \lesssim \Gamma \lesssim 30$.

\subsection{GRB 120404A \\ 6.3.1. GRB Properties}

GRB 120404A was detected and localized by the Swift BAT on 2012 April 04 at 12:51:02 UT (Stratta et al. 2012). The burst duration was $T_{90}=38.7 \pm 4.1 \mathrm{~s}$, with a fluence of $F_{\gamma}=$
$(1.6 \pm 0.1) \times 10^{-6} \mathrm{erg} \mathrm{cm}^{-2} \quad(15-150 \mathrm{keV}$ observer frame, Ukwatta et al. 2012). Swift and ground-based observatories detected an afterglow in the X-rays and UV/optical (Breeveld \& Stratta 2012; Gorbovskoy et al. 2012; Guidorzi et al. 2012; Xin et al. 2012b), as well as in the radio (Zauderer et al. 2012). Spectroscopic observations with Gemini-north yielded a redshift of $z=2.876$ (Cucchiara \& Tanvir 2012). The isotropic-equivalent $\gamma$-ray energy for this event is $E_{\gamma, \text { iso }}=(9 \pm 4) \times 10^{52} \mathrm{erg}\left(1-10^{4} \mathrm{keV}\right.$, rest frame; Guidorzi et al. 2014).

This burst has been previously studied in detail by Guidorzi et al. (2014), who interpret the optical re-brightening starting around $800 \mathrm{~s}$ in their well-sampled, multi-band optical light curves as due to the passage of the characteristic synchrotron frequency, $\nu_{\mathrm{m}}$. They additionally invoke RS emission to explain the flat $\left(t^{0.0 \pm 0.1}\right)$ portion of the optical light curve before the onset of the re-brightening. In the following, we propose an alternate model for the multi-band radio through $\mathrm{X}$-ray light curves in the context of energy injection.

The X-ray light curve before $700 \mathrm{~s}$ can be modeled as a power-law decay with $\alpha_{\mathrm{X}} \approx-2$. This light curve phase is likely part of the high latitude emission, and we ignore the data before $0.008 \mathrm{~d}$ in our analysis. The X-ray photon index at $0.12-0.24 \mathrm{~d}, \Gamma_{X}=2.3 \pm 0.3$ (Guidorzi et al. 2014), implying a spectrum, $F_{\nu} \propto \nu^{-1.3 \pm 0.3}$, is consistent with the spectral slope between the optical $i^{\prime}$-band and the X-rays at $0.07 \mathrm{~d}, \beta_{\text {opt }-\mathrm{X}}=-0.91 \pm 0.04$, suggesting that the optical and $\mathrm{X}$-ray bands are on the same segment of the afterglow SED, although the large uncertainty in $\beta_{X}$ leaves open the possibility that $\nu_{\mathrm{c}}$ lies between the optical and X-rays. Additionally, the spectral slope within the optical ( $B$ - to $i^{\prime}$-band) is $\beta_{\mathrm{opt}}=-1.3 \pm 0.2$ at $0.07 \mathrm{~d}$, indicating that extinction is present. The spectral index between the 19.2 and $24.5 \mathrm{GHz}$ observations at $0.75 \mathrm{~d}$ is $\beta_{\text {radio }} \approx 2$, which indicates that $\nu_{\mathrm{a}}$ is located above $24.5 \mathrm{GHz}$ at this time (Figure 17). 

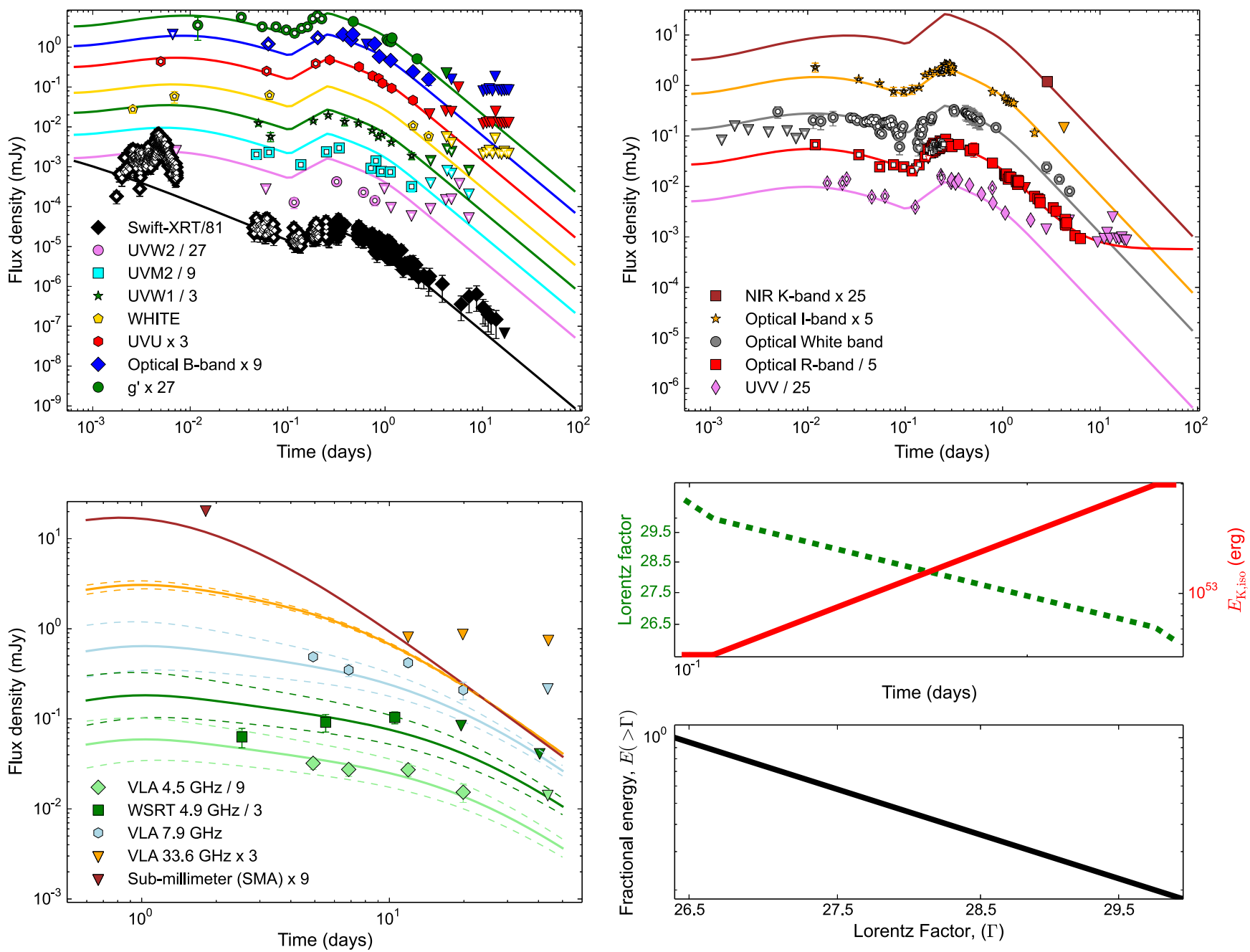

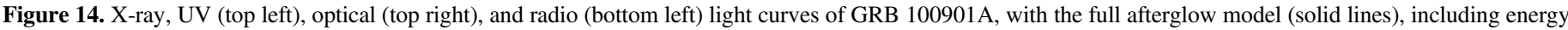

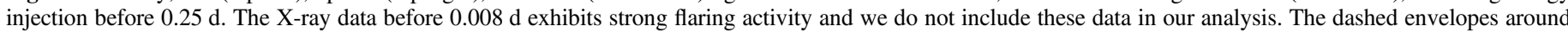

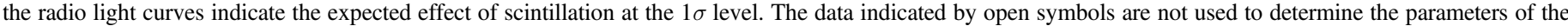

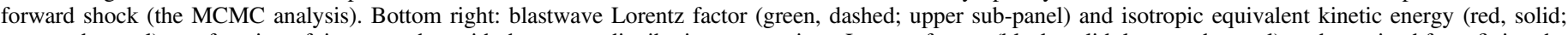

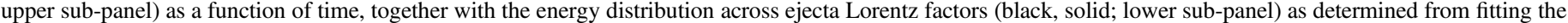
$\mathrm{X}$-ray/UV/optical re-brightening at $0.36 \mathrm{~d}$.

The optical $R$-band light curve declines as $t^{-1.9 \pm 0.02}$ after $0.13 \mathrm{~d}$, consistent with the X-ray decline rate of $t^{-1.8 \pm 0.1}$ after $0.05 \mathrm{~d}$. The steep decline of $\alpha \approx-2$ is indicative of a jet break before $\approx 0.1 \mathrm{~d}$. A broken power-law fit to the $B$-band light curve results in the parameters $t_{\mathrm{b}}=(2.8 \pm 0.4) \times 10^{-2} \mathrm{~d}$, $F_{\nu, \mathrm{B}}\left(t_{\mathrm{b}}\right)=252 \pm 10 \mu \mathrm{Jy}, \alpha_{1}=1.74 \pm 0.58, \alpha_{2}=-1.71 \pm$ 0.17 , and $y=0.78 \pm 0.43$, making this the earliest re-brightening episode of the four events studied in this paper.

\subsubsection{Forward Shock Model at $t \gtrsim 0.04 d$}

We interpret the optical light curve peaks at around $2500 \mathrm{~s}$ as the end of a period of energy injection, after which the afterglow evolves according to the standard framework with a

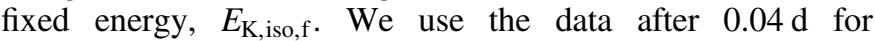
estimating the parameters of the blastwave shock and employ our MCMC tools described in Section 4 to model the afterglow after this time.

The parameters of our highest likelihood ISM model are $p \approx 2.06, \epsilon_{\mathrm{e}} \approx 0.27, \epsilon_{B} \approx 0.16, n_{0} \approx 2.8 \times 10^{2} \mathrm{~cm}^{-3}, E_{\mathrm{K} \text {,iso }} \approx$ $1.2 \times 10^{53} \mathrm{erg}, t_{\text {jet }} \approx 6.6 \times 10^{-2} \mathrm{~d}$, and $A_{\mathrm{V}} \approx 0.13 \mathrm{mag}$. The
Compton $y$-parameter for this model is $\approx 0.9$. The blastwave Lorentz factor is $\Gamma=4.1(t / 1 \mathrm{~d})^{-3 / 8}$ and the jet opening angle is $\theta_{\text {jet }} \approx 3: 1$. The beaming-corrected kinetic energy is $E_{\mathrm{K}} \approx 1.7 \times 10^{50} \mathrm{erg}$, while the beaming corrected $\gamma$-ray energy is $E_{\gamma} \approx 1.2 \times 10^{50} \mathrm{erg}\left(1-10^{4} \mathrm{keV}\right.$; rest frame). The MCMC analysis yields an isotropic equivalent kinetic energy of $E_{\mathrm{K}, \text { iso }}=1.3_{-0.2}^{+0.4} \times 10^{53} \mathrm{erg}$, which is similar to the value of $1.9_{-0.1}^{+0.7} \times 10^{53}$ erg derived by Guidorzi et al. (2014). The high circumburst density of $\log \left(n_{0}\right)=2.5_{-0.3}^{0.4}$ is also consistent with the value of $\log \left(n_{0}\right)=2.4_{-0.2}^{+0.02}$ determined by Guidorzi et al. 2014), and is driven by the low flux density and steep spectrum in the radio. However, unlike Guidorzi et al. (2014), our low value of $p \approx 2.1$ allows us to match the NIR to optical SED and the normalization of the X-ray light curve. We note that the high circumburst density also results in a low cooling frequency ${ }^{19}$ : in our highest likelihood model $\nu_{\mathrm{c}}<\nu_{\mathrm{a}}$ and the spectrum remains in the fast cooling regime through the

\footnotetext{
${ }^{19}$ Once again, $\nu_{\mathrm{c}}=\sqrt{\nu_{3} \nu_{11}}$, with $\nu_{3} \approx 8.0 \times 10^{10} \mathrm{~Hz}$, and $\nu_{11} \approx 5.0 \times$ $10^{10} \mathrm{~Hz}$ at $1 \mathrm{~d}$ (see footnote 12).
} 

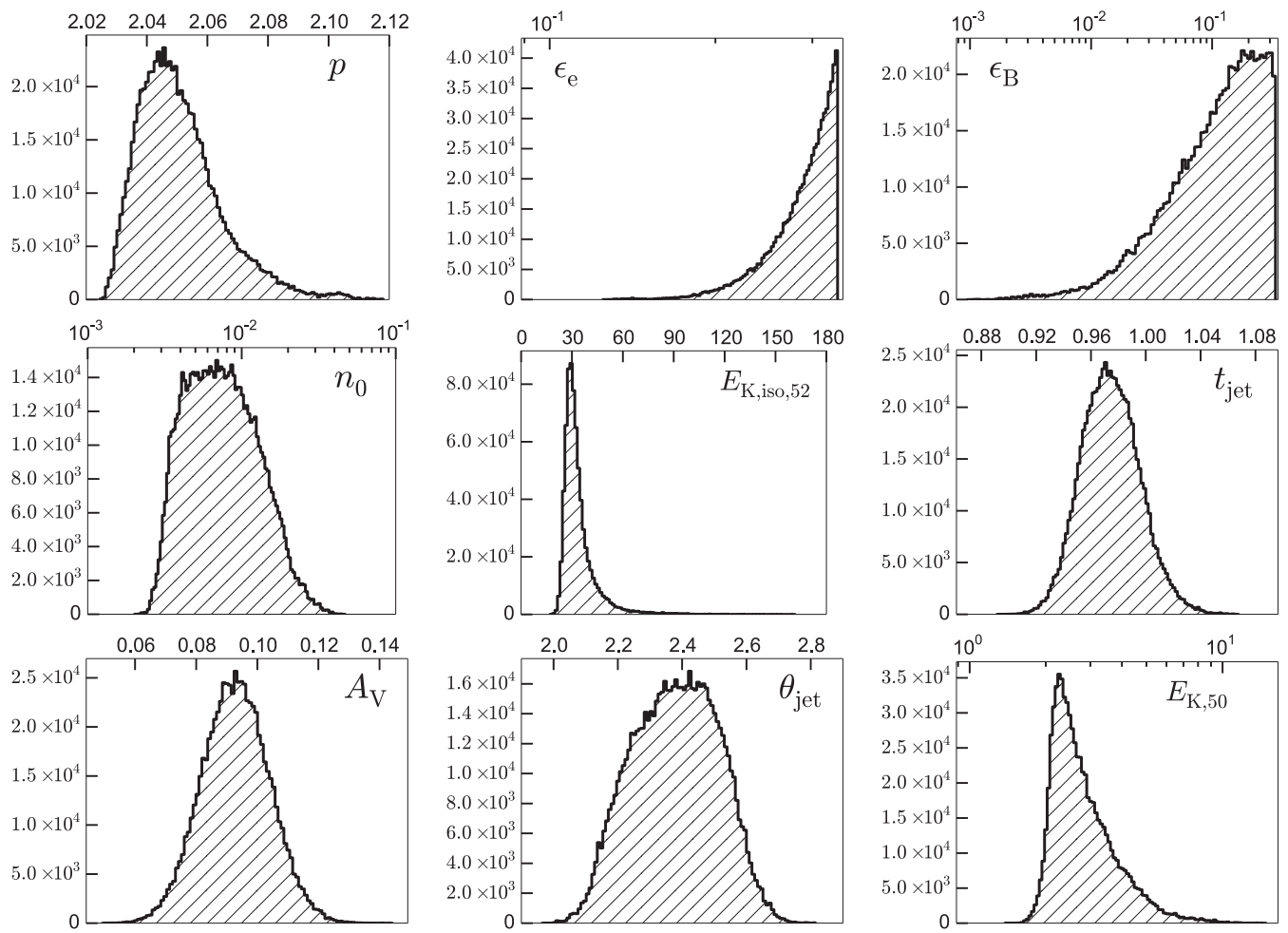

Figure 15. Posterior probability density functions for the physical parameters for GRB 100901A in a constant density environment from MCMC simulations. We

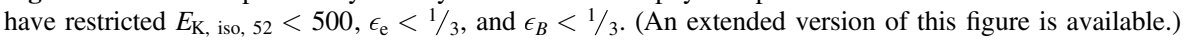
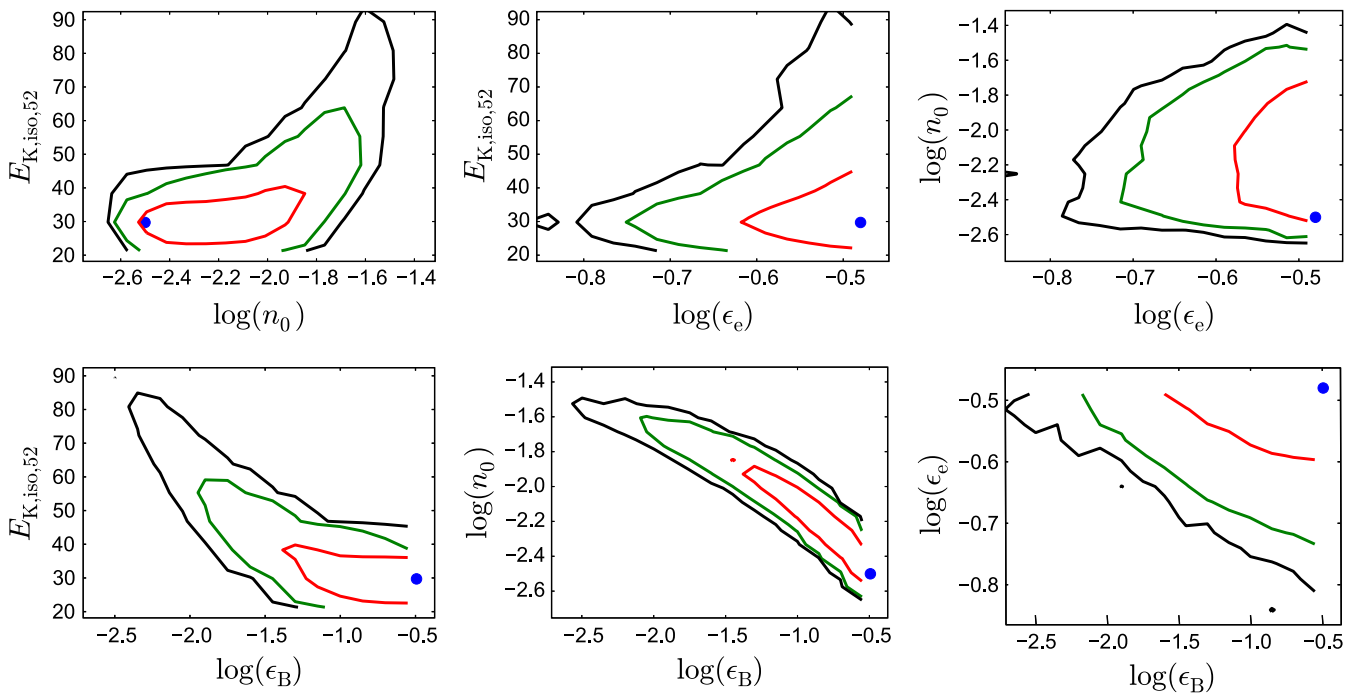

Figure 16. $1 \sigma$ (red), $2 \sigma$ (green), and $3 \sigma$ (black) contours for correlations between the physical parameters, $E_{\mathrm{K} \text {,iso, }}, n_{0}, \epsilon_{\mathrm{e}}$, and $\epsilon_{B}$ for GRB $100901 \mathrm{~A}$, in the ISM model from Monte Carlo simulations. We have restricted $\epsilon_{\mathrm{e}}<1 / 3$ and $\epsilon_{B}<1 / 3$. See the online version of this figure for additional plots of correlations between these

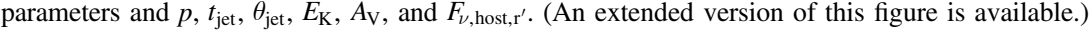

duration of the observations (spectrum 4 of Granot \& Sari 2002), with $\nu_{\mathrm{ac}} \approx 5.9 \times 10^{10} \mathrm{~Hz}, \nu_{\mathrm{sa}} \approx 2.0 \times 10^{11} \mathrm{~Hz}$, $\nu_{\mathrm{m}} \approx 9.1 \times 10^{11} \mathrm{~Hz}$, and $\nu_{\mathrm{c}} \approx 6.3 \times 10^{10} \mathrm{~Hz}$ at $1 \mathrm{~d}$.

We plot the posterior density functions for the all parameters in Figure 18 and correlation contours in Figure 19; we list our measured values for the physical parameters in Table 5. In the wind and ISM models, light curves at all frequencies become indistinguishable following a jet break. Since the jet break occurs early, soon after the start of the data used for deriving the parameters of the blastwave, we expect a viable wind model to exist as well. We discuss this model in Appendix E.

\subsubsection{Energy Injection Model}

We model the light curves before $t_{0}=0.04 \mathrm{~d}$ by injection of energy into the blastwave shock. We use the afterglow parameters for the highest-likelihood model (Section 6.3.2) as the final parameters following the end of energy injection, with $E_{\mathrm{K}, \text { iso,f }}=1.2 \times 10^{53} \mathrm{erg}$. We find that the optical and X-ray 

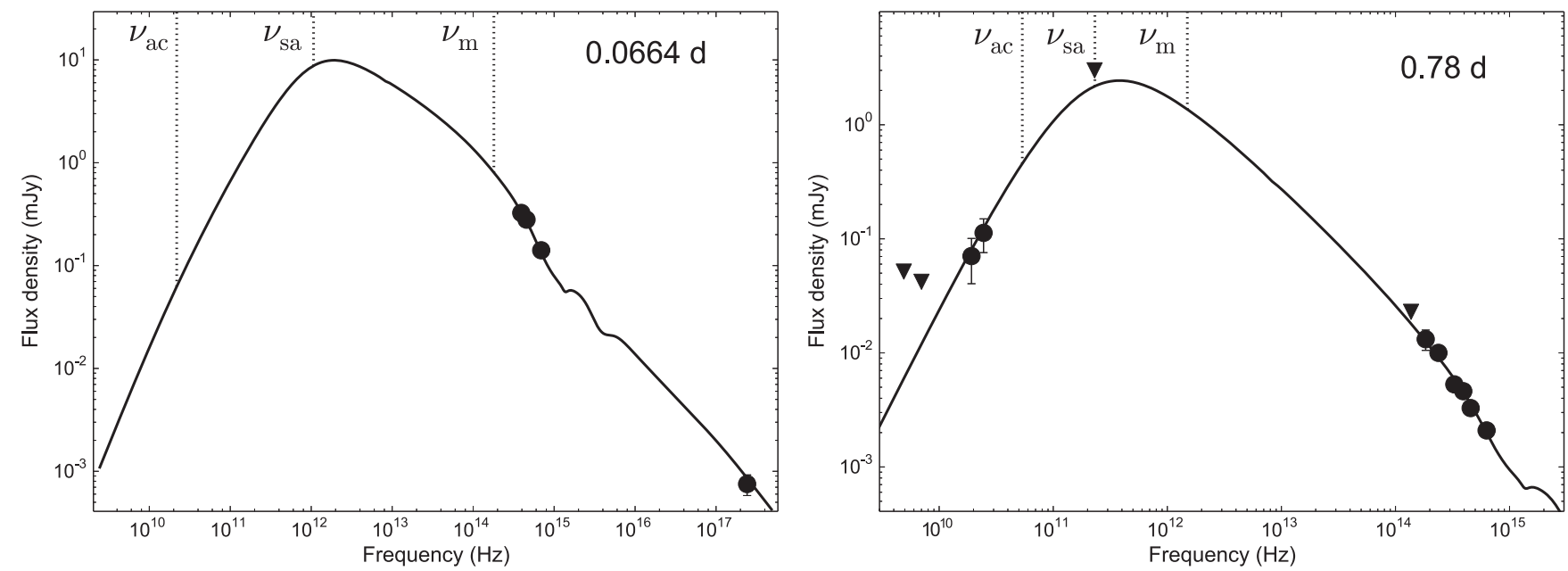

Figure 17. Left: observer-frame SED of the afterglow of GRB 120404A at 0.0664 d, together with the best-fit model (spectrum 4 from GS02: black, solid). Right: the same as the left panel, but at $0.78 \mathrm{~d}$. The radio detections with a steeply rising spectrum suggest that the self-absorption frequency is near or above the radio band, requiring a high circumburst density. Both SEDs are corrected for the effect of inverse Compton cooling, which is significant, with the Compton $y$-parameter $\approx 0.9$ during the fast cooling phase. We do not show the inverse Compton radiation, since even at its peak (near the Swift/XRT band), it is four orders of magnitude weaker than the synchrotron component. See Appendix A for a discussion of the inverse Compton effect in the context of modeling GRB afterglows.
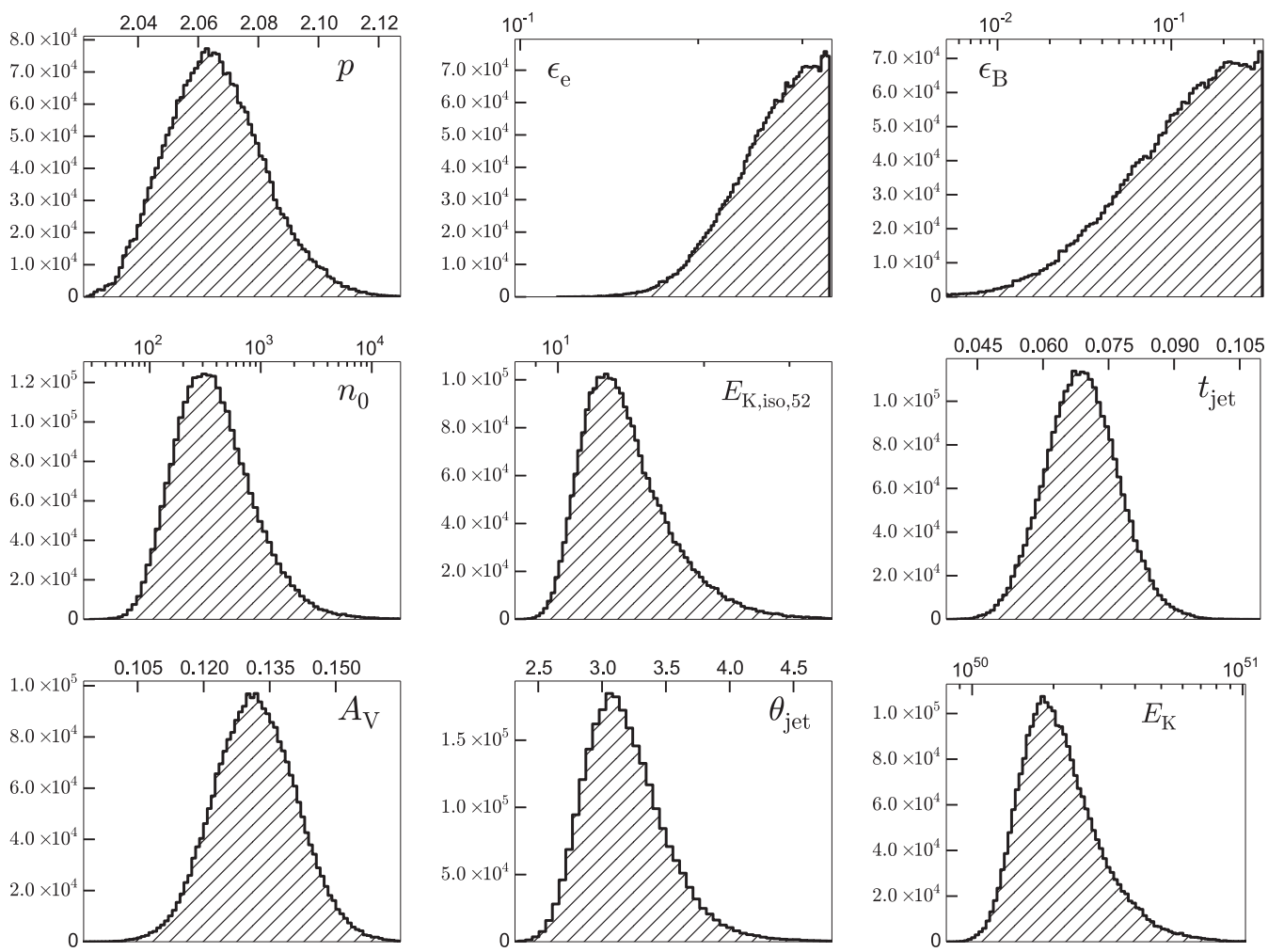

Figure 18. Posterior probability density functions for the physical parameters for GRB 120404A in a constant density environment from MCMC simulations. We have restricted $E_{\mathrm{K}, \text { iso }, 52}<500, \epsilon_{\mathrm{e}}<1 / 3$, and $\epsilon_{B}<1 / 3$. 

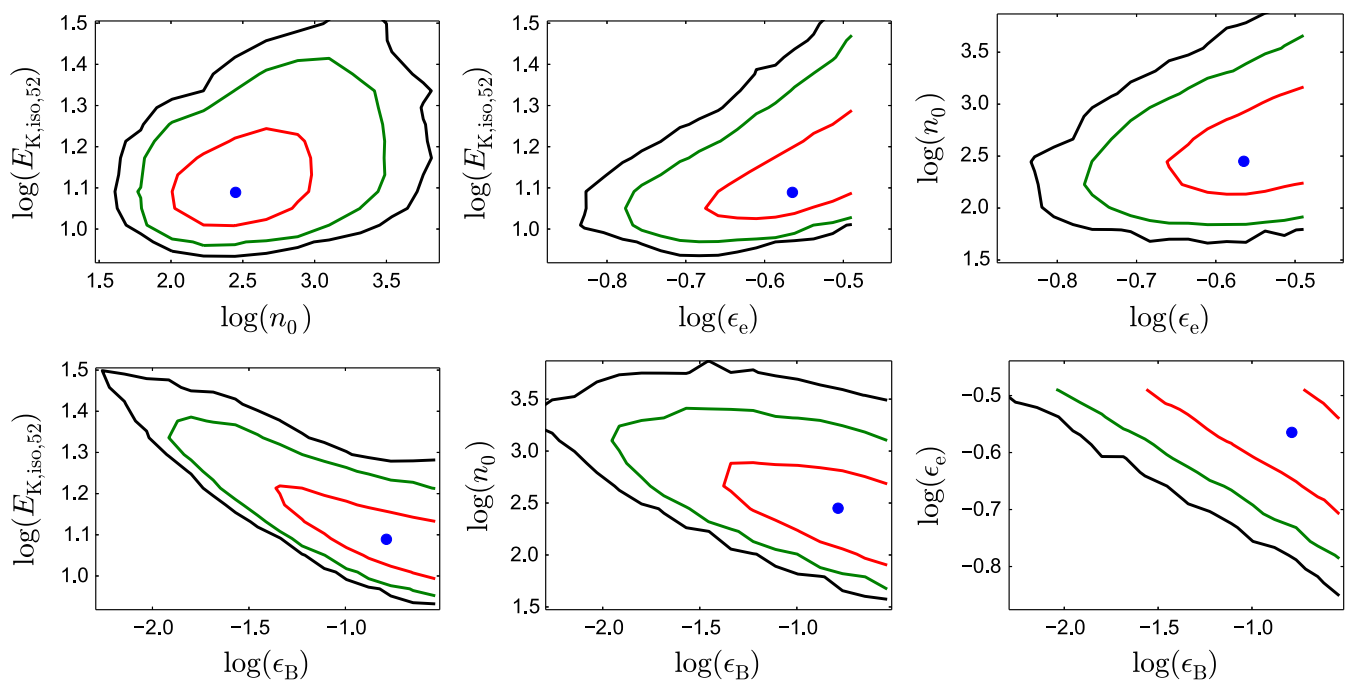

Figure 19. $1 \sigma$ (red), $2 \sigma$ (green), and $3 \sigma$ (black) contours for correlations between the physical parameters, $E_{\mathrm{K}, \text { iso }}, n_{0}, \epsilon_{\mathrm{e}}$, and $\epsilon_{B}$ for GRB $120404 \mathrm{~A}$, in the ISM model from Monte Carlo simulations. We have restricted $E_{\mathrm{K}, \text { iso,52 }}<500, \epsilon_{\mathrm{e}}<1 / 3$, and $\epsilon_{B}<1 / 3$. See the online version of this figure for additional plots of correlations between these parameters and $p, t_{\mathrm{jet}}, \theta_{\mathrm{jet}}, E_{\mathrm{K}}$, and $A_{\mathrm{V}}$. (An extended version of this figure is available.)
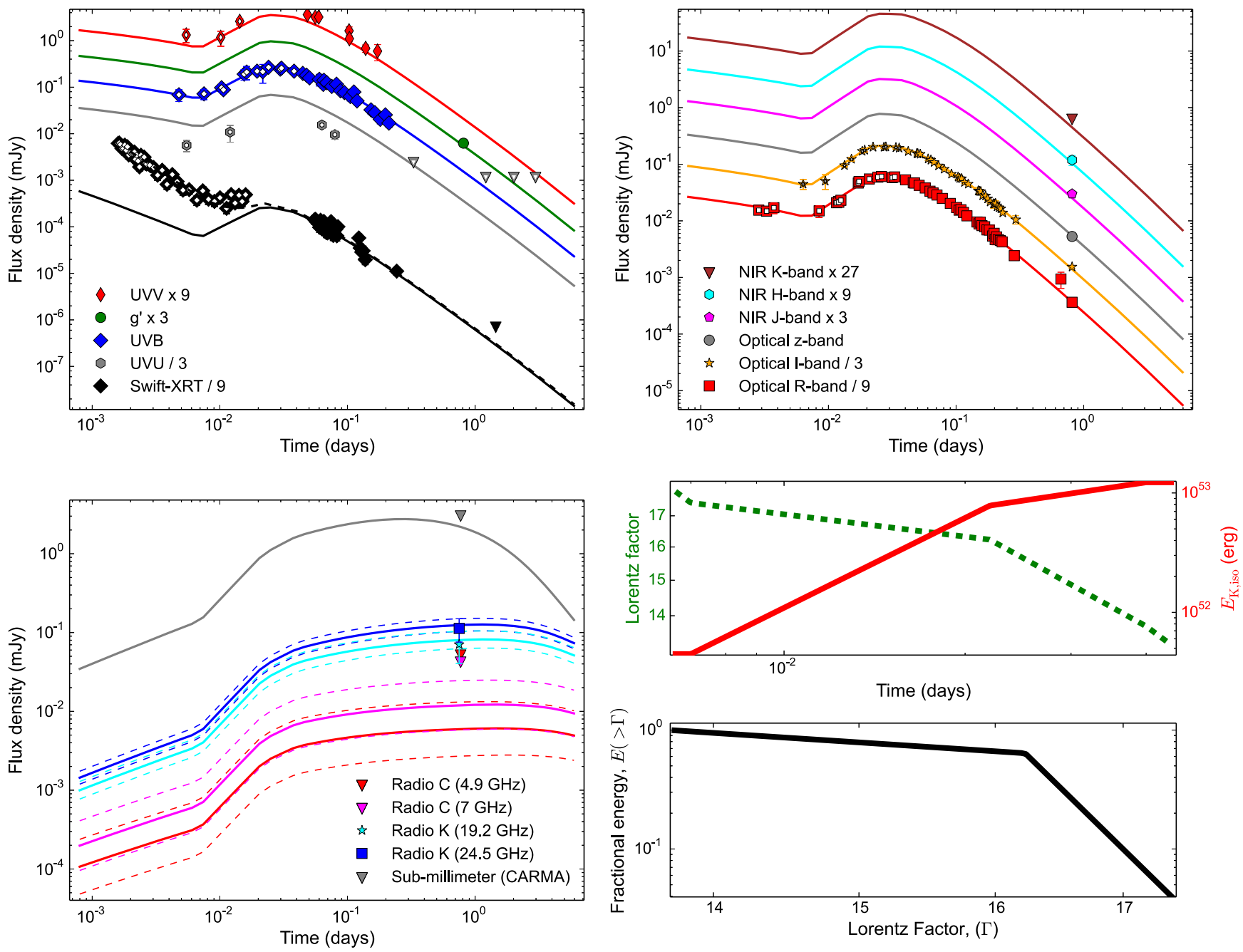

Figure 20. X-ray, UV (top left), optical (top right), and radio (bottom left) light curves of GRB 120404A, with the full afterglow model (solid lines), including energy injection before $0.04 \mathrm{~d}$. The X-ray data before $0.008 \mathrm{~d}$ is likely dominated by high-latitude prompt emission and we do not include these data in our analysis; the bestfit power law to the X-ray data before $0.008 \mathrm{~d}$ added to the blastwave model is shown in the upper left panel (black, dashed). The dashed envelopes around the radio light curves indicate the expected effect of scintillation at the $1 \sigma$ level. The data indicated by open symbols are not used to determine the parameters of the forward shock (the MCMC analysis). The $U$-band data are strongly affected by IGM absorption and are not included in the fit. Bottom right: blastwave Lorentz factor (green, dashed; upper sub-panel) and isotropic equivalent kinetic energy (red, solid; upper sub-panel) as a function of time, together with the energy distribution across ejecta Lorentz factors (black, solid; lower sub-panel) as determined from fitting the X-ray/UV/optical re-brightening at $0.04 \mathrm{~d}$. 

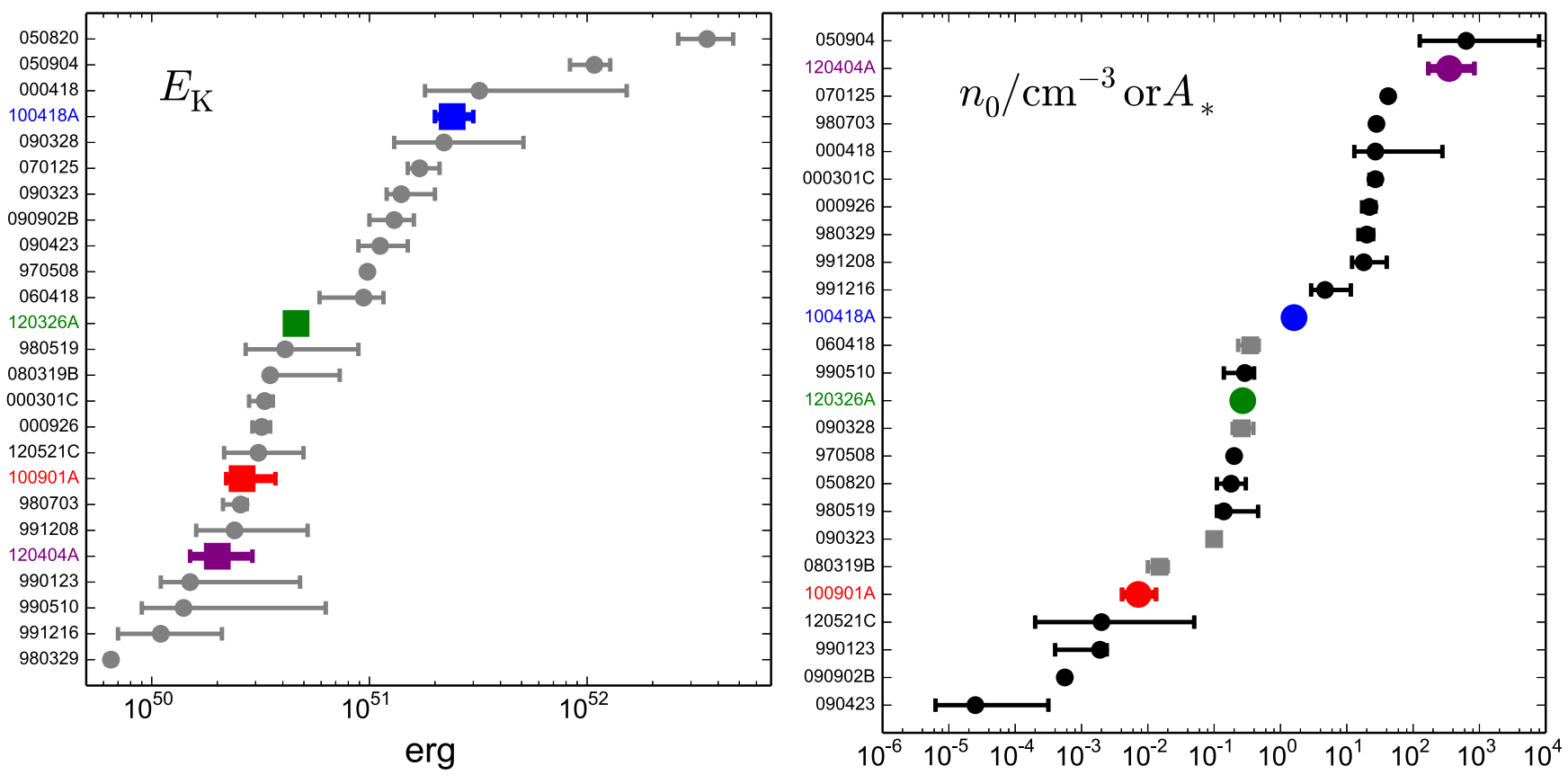

Figure 21. Beaming-corrected kinetic energy (left) and circumburst density (right) for both ISM (black circles) and wind-like environments (gray squares). The four GRBs in our analysis 100418A (blue), 100901A (red), 120326AA (green), and 120404A (purple), do not appear distinct from the comparison sample (gray and black; Panaitescu \& Kumar 2002; Yost et al. 2003; Chandra et al. 2008; Cenko et al. 2010, 2011; Laskar et al. 2014).

light curves can be modeled well by two subsequent periods of energy injection, beginning at $t_{2}=7 \times 10^{-3} \mathrm{~d}$ (Figure 20):

$$
\begin{aligned}
& E_{\mathrm{K}, \text { iso }}(t) \\
& = \begin{cases}E_{\mathrm{K}, \text { iso }, \mathrm{f}}, & t>t_{0}=0.04 \mathrm{~d} \\
E_{\mathrm{K}, \text { iso }, \mathrm{f}}\left(\frac{t}{t_{0}}\right)^{0.75}, & t_{1}=2.2 \times 10^{-2} \mathrm{~d}<t<t_{0} \\
E_{\mathrm{K}, \text { iso }, \mathrm{f}}\left(\frac{t_{1}}{t_{0}}\right)^{0.75}\left(\frac{t}{t_{1}}\right)^{2.5}, & t_{2}=7.0 \times 10^{-3} \mathrm{~d}<t<t_{1} \\
E_{\mathrm{K}, \text { iso }, \mathrm{f}}\left(\frac{t_{1}}{t_{0}}\right)^{0.75}\left(\frac{t_{2}}{t_{1}}\right)^{2.5}, & t<t_{2} .\end{cases}
\end{aligned}
$$

In this model, $E_{\mathrm{K} \text {,iso }}$ increases by a factor of $\approx 18$ from $4.4 \times 10^{51} \mathrm{erg}$ to $7.8 \times 10^{52} \mathrm{erg}$ between $7.0 \times 10^{-3} \mathrm{~d}$ and $2.2 \times 10^{-2} \mathrm{~d}$, and then by $\approx 50 \%$ to $E_{\mathrm{K} \text {,iso, } \mathrm{f}} \approx 1.2 \times 10^{53} \mathrm{erg}$ between $2.2 \times 10^{-2} \mathrm{~d}$ and $0.04 \mathrm{~d}$. Thus $\approx 95 \%$ of the final kinetic energy is injected into the blastwave between $7.0 \times 10^{-3} \mathrm{~d}$ and $0.04 \mathrm{~d}$. In comparison, $E_{\gamma, \text { iso }} \approx 10^{53} \mathrm{erg}$ is similar to the final kinetic energy. The blastwave Lorentz factor decreases from $\Gamma \approx 17.4$ at $7.3 \times 10^{-3}$ to $\Gamma \approx 16.2$ at $2.2 \times 10^{-2} \mathrm{~d}$, and then to $\Gamma \approx 13.7$ at the end of energy injection at $0.04 \mathrm{~d}$. The value of $m$ derived above corresponds to $s \approx 3.7$ for $13.7 \lesssim \Gamma \lesssim 16.2$ and $s \approx 40$ for $16.2 \lesssim$ $\Gamma \lesssim 17.4$

\section{DISCUSSION}

\subsection{Parameter Distributions}

We now turn to the question of how GRBs that exhibit simultaneous optical and X-ray re-brightening episodes compare with each other, and with events that do not exhibit such a feature. For this purpose, we use the compilation of measurements of $E_{\gamma}, \theta_{\text {jet }}, E_{\mathrm{K}}$, and $n_{0}$ (or $A_{*}$ ) from the literature (Panaitescu \& Kumar 2002; Yost et al. 2003; Friedman \&
Bloom 2005; Ghirlanda et al. 2007; Cenko et al. 2010, 2011) reported in Laskar et al. (2014). This sample includes GRBs from the pre-Swift era, as well as Swift and Fermi events.

The radio to X-ray observations of all four GRBs presented here can be fit by constant density ISM models. The best-fit models yield densities from $\approx 3 \times 10^{-3}$ to $\approx 3 \times 10^{2} \mathrm{~cm}^{-3}$ and final beaming-corrected kinetic energies from $\approx 2 \times 10^{50} \mathrm{erg}$ to $\approx 2 \times 10^{51} \mathrm{erg}$, spanning the full range of values inferred from GRB afterglow modeling (Figure 21). We constrain the jet break time and hence the opening angle of the GRB jet in each case, and find $\theta_{\text {jet }} \approx 2^{\circ}-21^{\circ}$, spanning the range inferred from the comparison sample.

The beaming-corrected $\gamma$-ray energies in the $1-10^{4} \mathrm{keV}$ restframe energy band of the events in our sample range from $5 \times 10^{49} \mathrm{erg}$ to $1.3 \times 10^{50} \mathrm{erg}$, while the median ${ }^{20}$ beaming corrected $\gamma$-ray energy of the comparison sample is $E_{\gamma}=\left(8.1_{-4.1}^{+3.1}\right) \times 10^{50} \mathrm{erg}(95 \%$ confidence interval, Figure 22$)$. Therefore, the observed values of $E_{\gamma}$ for the events in our sample are all smaller than the best estimate for the median of the comparison sample.

To further quantify this effect, we compute the radiative efficiency, $\eta \equiv E_{\mathrm{K}} /\left(E_{\gamma}+E_{\mathrm{K}}\right)$ for each $\mathrm{GRB}$ and in the comparison sample. Since both $E_{\mathrm{K}}$ and $E_{\gamma}$ have associated uncertainties, while the expression for $\eta$ is nonlinear in these two quantities, a proper accounting of the final uncertainty requires a Monte Carlo analysis. We generate $10^{5}$ Monte Carlo realizations of $E_{\mathrm{K}}$ and $E_{\gamma}$ for each burst, assuming a uniform distribution $^{21}$ between the $1 \sigma$ error bars. We then compute and

\footnotetext{
${ }^{20}$ The uncertainty on the median is computed using Greenwood's formula for the variance of the Kaplan-Meier estimate of the cumulative distribution function. This method accounts for both upper and lower limits, which exist in the data.

${ }^{21}$ We choose a uniform distribution instead of, say, a Gaussian distribution because the uncertainties on these parameters are frequently large compared to the mean, and a Gaussian distribution in linear space results in a tail of unphysical, negative values. A more detailed analysis requires the full posterior density functions for both $E_{\gamma}$ and $E_{\mathrm{K}}$ for every GRB, which are not available.
} 

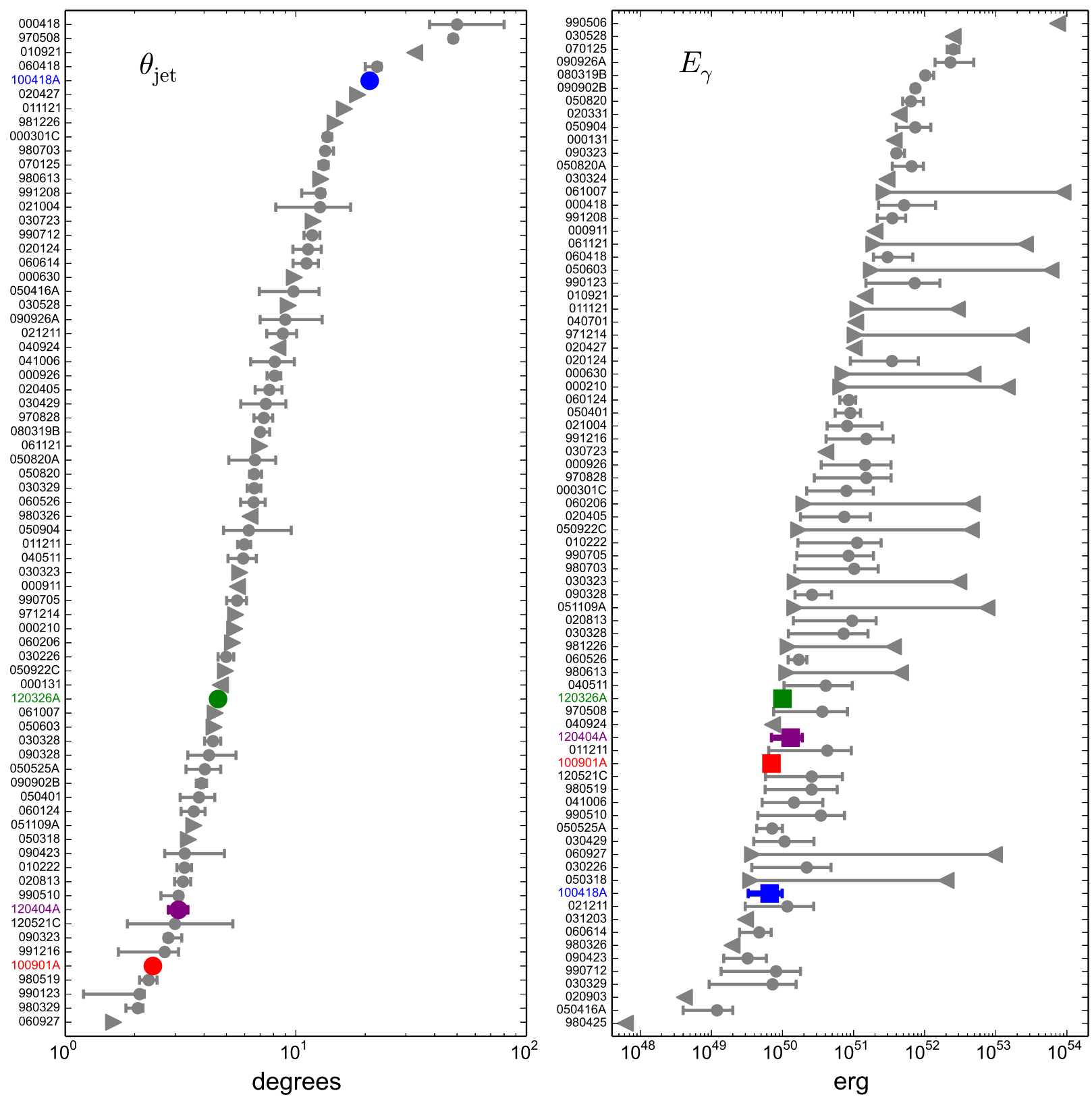

Figure 22. Beaming-corrected $\gamma$-ray energy (left) and jet opening angle (right) for the events in our analysis, GRB 100418A (blue), 100901A (red), 120326AA (green), and 120404A (purple), together with a comparison sample of long GRBs (gray; Friedman \& Bloom 2005; Ghirlanda et al. 2007; Cenko et al. 2010, 2011; Laskar et al. 2014). The isotropic-equivalent $\gamma$-ray energy for GRB 050904 is taken from Amati et al. (2008), and for GRB 090423 from Salvaterra et al. (2009). The four GRBs exhibiting X-ray and optical re-brightening episodes do not appear distinct from the comparison sample in $\theta_{\text {jet }}$, but appear to all reside at lower values of $E_{\gamma}$ than the median.

summarize the resulting distribution of $\eta$ using the median and $68 \%$ credible intervals (Figure 23). We find that the GRBs in our sample have systematically lower radiative efficiencies. This is consistent with the energy injection scenario, if the prompt $\gamma$-ray radiation is dominated by emission from the fastmoving ejecta, while a significant amount of kinetic energy is carried by slow-moving ejecta.

From this comparison, we conclude that events exhibiting simultaneous, multi-wavelength re-brightening episodes also span the same wide range of circumburst densities, jet opening angles, and beaming-corrected kinetic energies inferred from previous studies of events at $z \sim 1$ and $z \gtrsim 6$. However, the events reported here have smaller beaming-corrected $\gamma$-ray energies, and as a result lower radiative efficiencies than the comparison sample, suggesting that the prompt radiation is dominated by ejecta at high Lorentz factors, while the bulk kinetic energy is dominated by slow-moving ejecta at least in these cases.

\subsection{Absence of $R S$}

When energy injection into the blastwave is caused by a distribution of ejecta Lorentz factors, the RS from the initial interaction of the leading edge of the ejecta with the circumburst medium is expected to continue to propagate through the ejecta until the end of the period of energy injection (Sari \& Mészáros 2000). During the period of energy injection, the afterglow SED is expected to be composed of contributions 


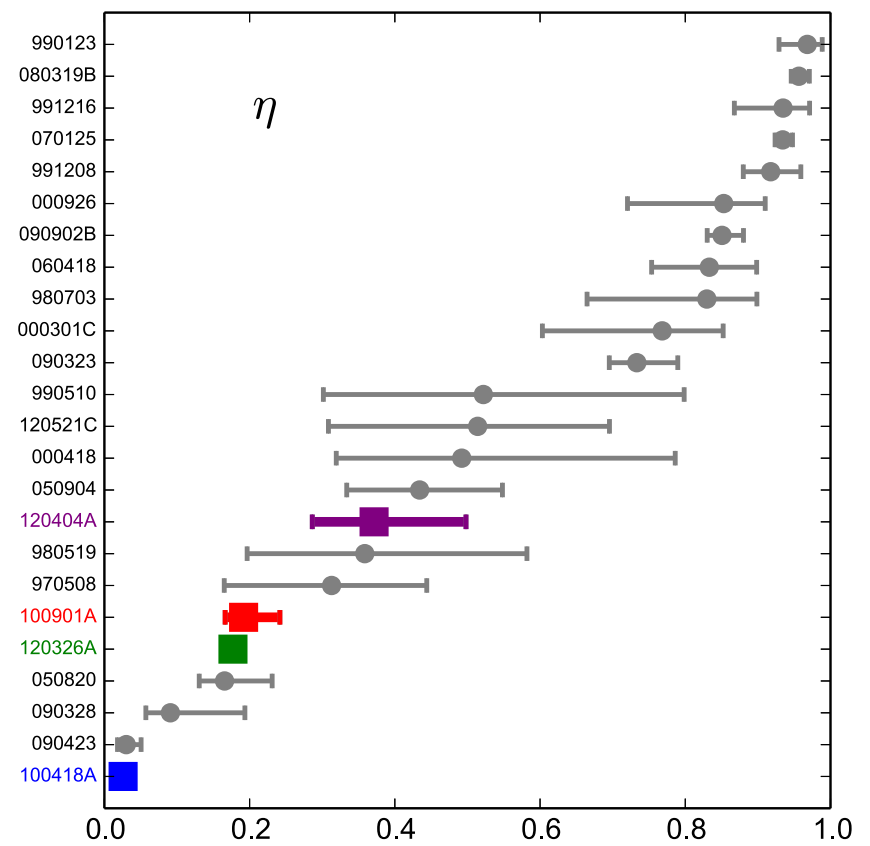

Figure 23. Radiative efficiency, $\eta$ for the events in our analysis, GRB 100418A (blue), 100901A (red), 120326AA (green), and 120404A (purple), together with a comparison sample of long GRBs (gray; Friedman \& Bloom 2005; Ghirlanda et al. 2007; Cenko et al. 2010, 2011; Laskar et al. 2014), with 68\% credible regions (error bars) about the median (points) computed from $E_{\mathrm{K}}$ and $E_{\gamma}$ using a Monte Carlo procedure. The four GRBs exhibiting X-ray and optical re-brightening episodes have lower radiative efficiencies than the median.

from both forward (FS) and reverse (RS) shocks, each with its three characteristic frequencies and flux normalizations. The spectral characteristics of the two shocks are expected to be related by $F_{\nu, \max , \mathrm{RS}}(t)=F_{\nu, \max , \mathrm{FS}}(t) \times \Gamma(t), \quad \nu_{\mathrm{m}, \mathrm{RS}}(t)=$ $\nu_{\mathrm{m}, \mathrm{FS}}(t) / \Gamma^{2}(t)$, and $\nu_{\mathrm{c}, \mathrm{RS}}(t)=\nu_{\mathrm{c}, \mathrm{FS}}(t)$, while the two selfabsorption frequencies should be related by $\nu_{\mathrm{a}, \mathrm{RS}}(t)$ $=\nu_{\mathrm{a}, \mathrm{FS}}(t) \times \Gamma^{\lambda}(t)$, where $\lambda=3 / 5$ when both shocks are fast cooling and $\lambda=8 / 5$ when both shocks are slow cooling. The RS is expected to last through the period of energy injection, whereupon all ejecta have been decelerated to a common Lorentz factor, and the two shocks decouple in their subsequent evolution.

A calculation of the RS SED based on the above considerations, together with the Lorentz factor of the blastwave at the end of the period of energy injection $\left(t_{\mathrm{dec}}\right)$ and the SED of the forward shock at that time indicates that the RS generically peaks around the millimeter band at $t_{\mathrm{dec}}$ (Sari \& Mészáros 2000). For GRB 100901A and 120404A, there are no data in the millimeter bands at the relevant time. For GRBs 120326A and $100418 \mathrm{~A}$ where we have millimeter observations at $\approx t_{\mathrm{dec}}$, we find that the RS light curves over-predict the observations by factors of $\approx 2-10$. Additionally, the RS would also contribute a flux density comparable to the forward shock in the X-rays and optical for all four events during energy injection, which would require suppressing the forward shock before the re-brightening by invoking even lower blastwave energies (and consequently requiring an even greater rate of energy injection) before the optical/X-ray peak. Thus the data are inconsistent with the presence of a strong RS for these events.

We note that in our model there is a gap before the beginning of energy injection. If the energy injection is caused by a shell of material with a distribution of Lorentz factors (the "injective shell") catching up with the initial shell ("the impulsive shell"), then a long-lasting RS in the injective shell is only expected when the two shells collide violently, with a relative Lorentz factor greater that the sound speed in the injective shell (Zhang \& Mészáros 2002). If the injective shell is released from the central engine at roughly the same time as the impulsive shell, the collision between the two will be mild, and a RS will not occur. This appears to be the case for the GRBs studied here, suggesting that the shell collisions in these events exhibiting $\mathrm{X}$-ray and UV/optical re-brightening episodes are gentle, resulting simply in a transfer of energy to the blastwave. Even more fundamentally, this implies that the shells are emitted at the same time from near the central engine and thus the engine need not be on during the re-brightening episode.

\subsection{Energy Injection: A Ubiquitous Phenomenon?}

The phenomenon of short-lived plateaus at an early time is ubiquitous in Swift/XRT afterglow light curves (Liang et al. 2007). Using a complete sample of Swift/XRT light curves through 2010, Margutti et al. (2013) find about 37\% of longduration GRBs exhibit a shallow decay phase, with $-1 \lesssim \alpha \lesssim 1$. If these plateaus are caused by injection of energy into the forward shock, the events with X-ray/optical re-brightenings discussed in this paper might be the extreme tail of a distribution in energy injection factor, duration or rate. A rigorous exploration of these possibilities requires multiwavelength fits to the data, but such data are generally not available. We therefore compute energy injection fractions based on the X-ray light curves alone, and compare the results for the objects where we do have multi-wavelength observations as reported in this paper.

Margutti et al. (2013) measured the timing of X-ray plateaus in Swift/XRT light curves and quantified them by their start time, $t_{1}$, end time, $t_{2}$, and rise rate ${ }^{22}$ during the plateau phase, $\alpha$. For our X-ray plateau analysis, we select the 96 events in their study where the reported uncertainty in $\alpha$ is $<0.3$. We repeat their light curve decomposition analysis on the X-ray light curves of GRB 120326A and GRB 120404A (which are not included in their sample), and find $t_{1}=5.7 \times 10^{-3} \mathrm{~d}$, $t_{2}=0.62 \mathrm{~d}$, and $\alpha=0.27 \pm 0.03$, for GRB 120326A and $t_{1}=9.8 \times 10^{-3} \mathrm{~d}, t_{2}=2.9 \times 10^{-2} \mathrm{~d}$, and $\alpha=0.76 \pm 0.23$ for GRB 120404A. ${ }^{23}$

We define the plateau duration, $\Delta T \equiv t_{2}-t_{1}$ and the fractional duration, $\xi \equiv\left(t_{2}-t_{1}\right) /\left(t_{2}+t_{1}\right) \in[0,1)$. Note that $\xi \rightarrow 1$ when $t_{2} \gg t_{1}$, which corresponds to the case that the plateau lasts much longer than its onset time. Margutti et al. (2013) computed the start time of the plateau using the intersection of the steep decay phase with the best-fit plateau model; hence they do not report an error on this quantity. We can estimate the uncertainty on $\xi$ using $\sigma_{\xi}=\sigma_{t_{2}} \partial \xi / \partial t_{2}=2 t_{1} \sigma_{t_{2}} /\left(t_{1}+t_{2}\right)^{2}$, which is dimensionless as desired.

Assuming the X-ray band is located above the cooling frequency for all cases (which is indeed the case for the five events considered in detail here), and that $p=2$, we can compute an effective energy injection rate, $E \propto t^{m}$, where $m=\alpha+1$ is the rate required to bring the measured light

\footnotetext{
${ }^{22}$ Margutti et al. (2013) use the convention $F(t) \propto t^{-\alpha}$, which is opposite to the convention in this paper.

${ }^{23}$ The X-ray light curve of GRB 120404A is not well-sampled near the peak of the re-brightening and we therefore fix the peak time in the fit to the value inferred from the optical light curve $(\approx 0.3 \mathrm{~d}$; Section 6.3.3).
} 


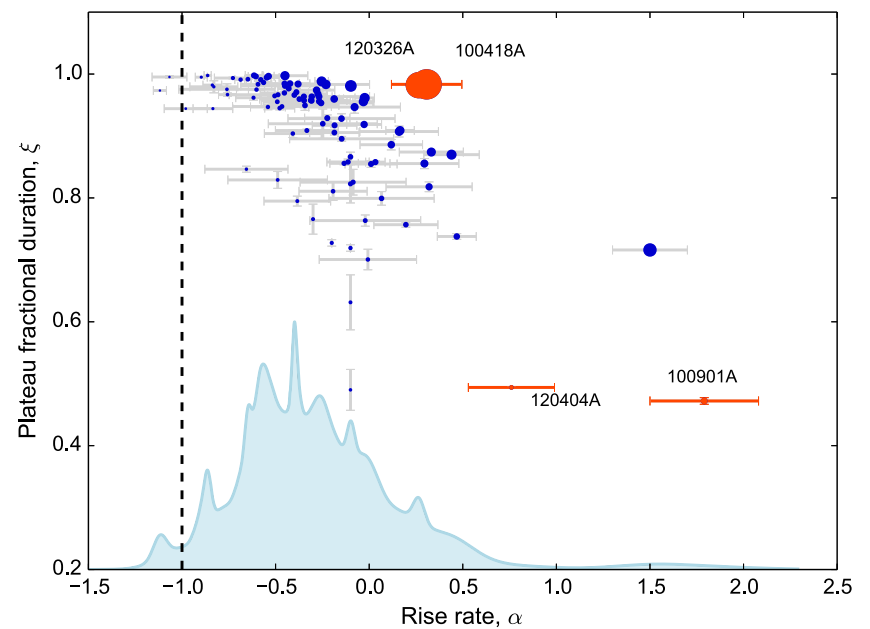

Figure 24. Fractional duration of X-ray plateaus from Margutti et al. (2013) as a function of the light curve rise rate, $\alpha$ (with the convention $F \propto t^{\alpha}$ ). Errors in both directions are computed according to the formulas described in Section 7.3. The vertical dashed black line indicates the canonical light curve decay of $\alpha \approx-1$ expected for $p \approx 2.21 \%$ of events with plateaus exhibit rebrightenings $(\alpha>0)$. Events analyzed in this paper are shown in red. The area of each symbol is linearly proportional to the ratio of the energy at the end to that at the beginning of the plateau phase, $\Upsilon$. The blue point at $\alpha \approx-1.5$ is GRB 081028, which also exhibits an X-ray/optical re-brightening; however, this event does not have radio data and we therefore exclude it from our multiwavelength analysis. A probability density function of the distribution of $\alpha$ (computed from a kernel density estimate using $\sigma_{\alpha}$ as a varying kernel bandwidth) is shown in cyan.

curve slope to $\alpha$ from the theoretically expected value ${ }^{24}$ of $t^{(2-3 p) / 4} \propto t^{-1}$ for no energy injection. For the sake of simplicity and uniformity, we use $p=2$ even where we have other measurements of $p$, such as for the events reported in this paper. The ratio of the energy at the end of the plateau phase to the energy at the beginning of the plateau phase is then given by $\Upsilon \equiv E_{2} / E_{1}=\left(t_{2} / t_{1}\right)^{m} .^{25}$

We plot the plateau fractional duration, $\xi$ against the plateau slope, $\alpha$ in Figure 24, scaling the area of the symbols by $\Upsilon$. The slopes during the plateau phase range from $\alpha \approx-1$ to $\approx 1.8$. As evident from the kernel density estimate of $\alpha$, most light curves exhibit a gentle decay during the plateau phase (Figure 24). However, all four events in our sample are exceptions to this rule. $^{26}$ These four events also exhibit the greatest rise in their plateau phase light curves for a given normalized duration, $\xi$. Two out of these four (GRBs 120326A and 100418A) have the largest fractional changes in the kinetic energy of the entire sample of 98 events. These are also the events with the largest value of $\xi$ in our sample. For GRB 120404A, we note that the $\mathrm{X}$-ray light curve around the peak of the re-brightening is missing due to a Swift orbital gap. Therefore any results that

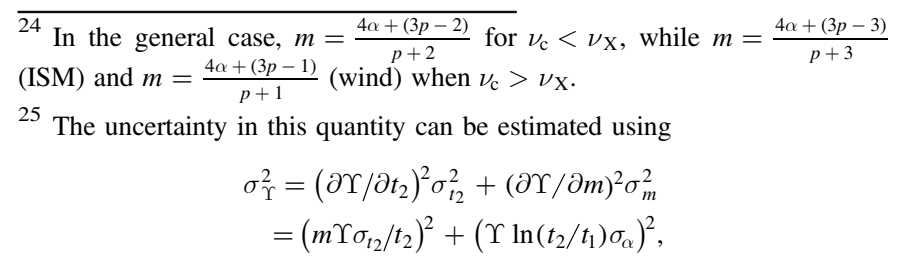

where we have taken $\sigma_{m}=\sigma_{\alpha}$. This assumes that $t_{2}$ and $\alpha$ are independent. However, these quantities are expected to be correlated, and a more complete analysis of the uncertainty in $\Upsilon$ requires the full covariance matrix between $\alpha$ and $t_{2}$.

${ }^{26}$ Since we selected GRBs that exhibit an X-ray re-brightening, this is partly by sample construction.

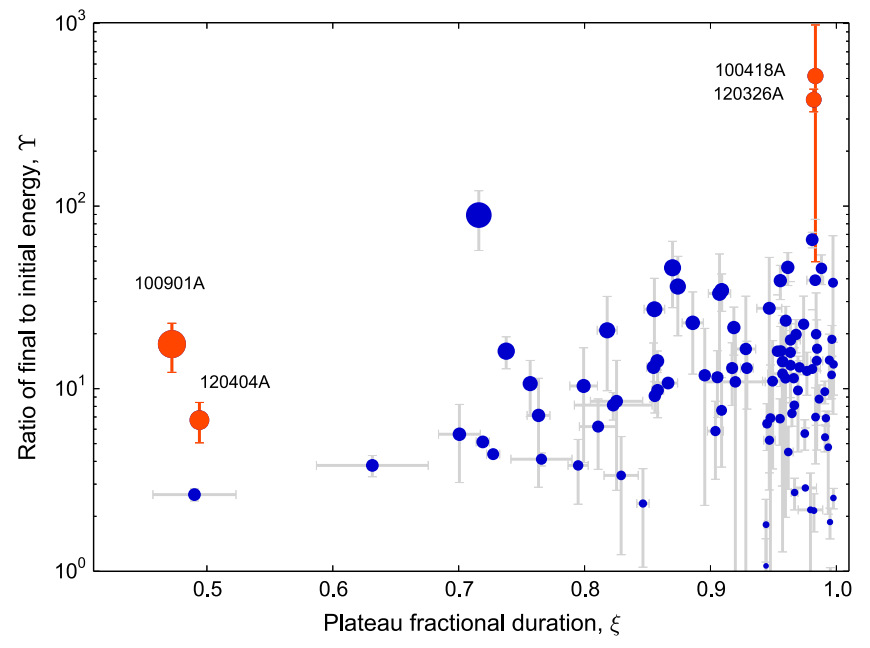

Figure 25. Ratio of energy at the end to that at the beginning of the plateau phase in X-ray light curves of 96 events from Margutti et al. (2013), as well as GRBs 120326A and 120404A from our analysis, as a function of the plateau fractional duration, $\xi$, with error bars computed according to the prescription in Section 7.3. The size of the symbols is scaled to a measure of the light curve rise rate during the plateau decay phase, $\alpha$, with the radius of the symbols proportional to $\alpha+1.5$. Larger symbols denote a greater departure from a canonical light curve decay of $\alpha \approx-1$, indicating greater rates of energy injection during the plateau phase.

rely solely on the X-ray data of this burst underestimate the value of $\alpha$ and the fraction of energy injection relative to the multi-wavelength analysis we carried out in Section 6.3.3.

In Figure 25 we plot the ratio of final to initial energy, $\Upsilon$ against the normalized plateau duration, $\xi$, scaling the radius ${ }^{27}$ of the symbols by $\alpha$. We find that for a given (normalized) plateau duration, the events in our sample have the largest fractional change in blastwave kinetic energy during the plateau phase. At the same time, they also possess the steepest rise rates in the sample, which is simply indicative of our selection criteria for inclusion in the present analysis. Finally, these events have the shortest normalized plateau durations for a given fractional change in energy. Together, these observations suggest that a large amount of injected energy is not sufficient to cause simultaneous X-ray/optical re-brightenings, but that it must be done in a relatively small amount of time, and thus that the defining characteristic of these events is a high rate of energy injection. Physically, this translates to a steep distribution of ejecta Lorentz factors over a small range of $\Gamma$. Hascoet et al. (2015) recently suggested that the interaction of low-Lorentz factor ejecta with the RS can explain flares in the X-ray light curves. Our observations provide supporting evidence for this hypothesis in the form of significant ejecta energy down to the requisite low Lorentz factors, $\Gamma \sim 10$.

\subsection{Energetics}

Having compared the plateau duration, light curve rise rate, and injected energy fraction for our sample with a complete sample of Swift events using the X-ray data alone, we now turn to an analysis of the results from our multi-wavelength energy injection modeling in this context. In Figure 26 we plot the

\footnotetext{
27 Since $\alpha$ can be negative while the area of the symbols in the plot is a positive definite quantity, we scale the radius of the symbols as $\alpha+1.5$, where the additive term accounts for the minimum value of $\alpha$.
} 


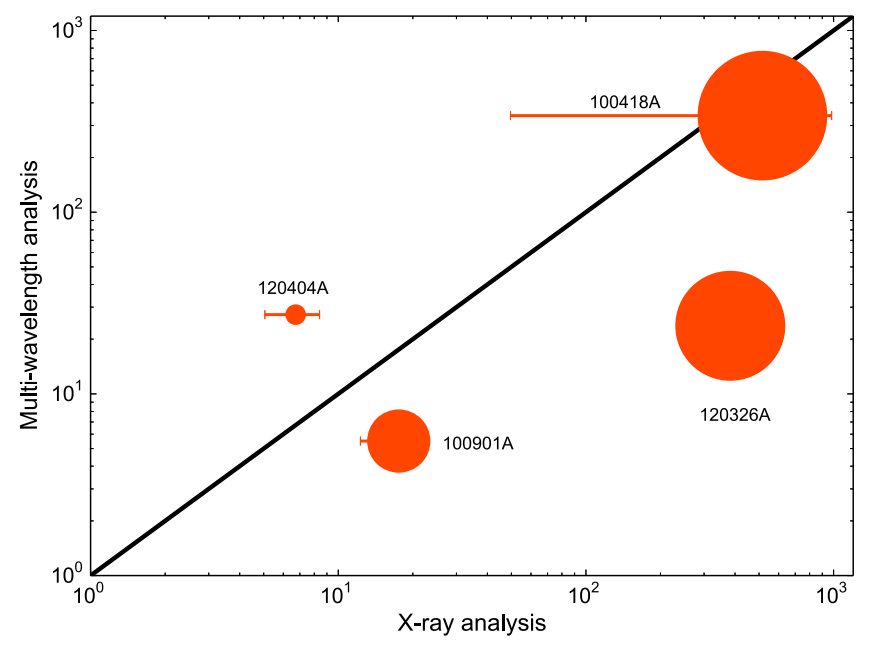

Figure 26. Ratio of final energy to the energy prior to the first injection episode from the multi-wavelength analysis ( $y$-axis) compared to the ratio of the energy at the end to that at the start of the "plateau phase," $\Upsilon$, computed in Section 7.3 for the five events in our sample, together with the 1:1 line (solid, black). The area of the symbols is linearly proportional to the physical duration of the plateaus, $t_{2}-t_{1}$

fractional change in energy determined from multi-wavelength modeling against the fractional change in energy, $\Upsilon$, inferred from the X-ray-only analysis in Section 7.3 for the four events in our sample. We scale the area of the symbols with the plateau physical duration, $t_{2}-t_{1}$. As expected, events with longer plateau durations have greater estimated energy injection fractions both from the X-ray analysis and from the full multi-wavelength study. The fractional change in energy from the X-ray analysis is higher than inferred from the full model for three out of four cases. This is due to the typically lower value of $m$ from the X-ray-only analysis compared to the full model. Ultimately, this can be traced to the fitting procedure: Margutti et al. (2013) fit a sum of a steep decay and a rising light curve, the sum of which results in the X-ray plateau, whereas we do not subtract fits to the steep decay phase from the X-ray light curve prior to our multi-band modeling. In the case of GRB 120326A, where the X-ray analysis over-estimates $\Upsilon$ by an order of magnitude, the variance between the two techniques is due to differences in the plateau start time $\left(4.3 \times 10^{-2} \mathrm{~d}\right.$ for the multi-wavelength analysis, compared to $5.7 \times 10^{-3} \mathrm{~d}$ in the X-ray analysis). Based on this comparison, we conclude that a determination of $\Upsilon$ using the X-ray data alone yields a reasonable estimate of the energy injection factor on average, but that multi-wavelength modeling is essential to obtain the full picture.

\section{SUMMARY AND CONCLUSIONS}

Plateaus and re-brightening episodes are frequent in GRB afterglow X-ray light curves. However, X-ray data by themselves provide only a limited understanding of the physical processes underlying these unexpected phenomena. We perform a thorough multi-wavelength study of all longduration GRBs through 2012 featuring simultaneous X-ray, and UV/Optical re-brightenings and that have radio detections, using a Markov Chain Monte Carlo analysis and a physical afterglow model. Our analysis yields the first set of models that explain the multi-wavelength afterglows for all of these events with the same unifying principle. In all cases, the afterglow light curves can be modeled with a standard forward shock model following the re-brightening episode, an ISM density profile, and a jet break. From our multi-wavelength analysis, we find that the circumburst densities, jet opening angles, and beaming-corrected kinetic energies for these events span the full range described by typical GRBs at $z \sim 1$ and $z \gtrsim 6$.

We explore a range of possible models to understand the rebrightenings, including the onset of the afterglow, off-axis viewing geometry, and continuous energy injection. We are able to rule out the afterglow onset and off-axis jets, and find instead that injection of energy into the blastwave (the so-called "refreshed-shock" scenario) provides a good explanation for all events. We interpret energy injection in the framework of the stratified Lorentz factor model, and find that our measured energy injection rates always obey the theoretical constraints relating the rate of injection and the distribution of ejecta Lorentz factors in the ISM model.

We perform the first measurement of the ejecta Lorentz factor distribution index, $s$, and find $s \approx 3-40$, suggesting that a large amount of kinetic energy resides in the slowest-moving ejecta. This is supported by low radiative efficiencies for the events in our sample, indicating that the $\gamma$-ray radiation is dominated by ejecta at high Lorentz factors, while the kinetic energy is dominated by slower-moving ejecta. We note that keeping the injection rates simple power laws allows us to directly convert the injection rate to a Lorentz factor distribution index, but the true injection rate and also the true Lorentz factor distribution is likely to be a smoother function of time and Lorentz factor, respectively.

Finally, using a compilation of X-ray plateaus in GRB afterglows, we present a comparative discussion of this interesting sub-population of GRBs. We find that the phenomenon of energy injection is ubiquitous in long-duration GRBs, with re-brightening episodes likely simply extreme injection events. In future work, we aim to fit the light curves before the re-brightening episodes in a statistical sense, allowing us to estimate uncertainties on the rate and duration of the energy injection episodes. At the same time, radio monitoring of Swift events exhibiting re-brightening episodes will be crucial in multi-wavelength modeling of this interesting class of GRBs, while ALMA observations will irrefutably establish the presence or absence of RSs, laying to rest the question of whether the energy injection process is violent or gentle.

We thank the anonymous referee for their thoughtful and detailed comments. We thank M. Viero and J. Viera for acquiring the P200 WIRC observations as part of our target-ofopportunity program. Support for CARMA construction was derived from the states of California, Illinois, and Maryland, the James S. McDonnell Foundation, the Gordon and Betty Moore Foundation, the Kenneth T. and Eileen L. Norris Foundation, the University of Chicago, the Associates of the California Institute of Technology, and the National Science Foundation. Ongoing CARMA development and operations are supported by the National Science Foundation under a cooperative agreement, and by the CARMA partner universities. The Submillimeter Array is a joint project between the Smithsonian Astrophysical Observatory and the Academia Sinica Institute of Astronomy and Astrophysics and is funded by the Smithsonian Institution and the Academia Sinica. The National Radio Astronomy Observatory is a facility of the 
Table 10

Inverse Compton Corrections to Spectral Break Frequencies

\begin{tabular}{lcccc}
\hline \hline $\begin{array}{l}\text { Spectral } \\
\text { Break }\end{array}$ & $\begin{array}{c}\text { Break } \\
\text { Frequency }\end{array}$ & Break Type & $\begin{array}{c}\text { Frequency } \\
\text { Scaling }\end{array}$ & $\begin{array}{c}\text { Flux Density } \\
\text { Scaling }\end{array}$ \\
\hline 1 & $\nu_{\mathrm{sa}}$ & Self-absorption & $(1+Y)^{0}$ & $(1+Y)^{0}$ \\
2 & $\nu_{\mathrm{m}}$ & Characteristic & $(1+Y)^{0}$ & $(1+Y)^{0}$ \\
3 & $\nu_{\mathrm{c}}$ & Cooling & $(1+Y)^{-2}$ & $(1+Y)^{p-1}$ \\
4 & $\nu_{\mathrm{m}}$ & Characteristic & $(1+Y)^{0}$ & $(1+Y)^{0}$ \\
5 & $\nu_{\mathrm{sa}}$ & Self-absorption & $(1+Y)^{0}$ & $(1+Y)^{0}$ \\
6 & $\nu_{\mathrm{sa}}$ & Self-absorption & $(1+Y)^{-2} p$ & $(1+Y)^{-5}$ \\
7 & $\nu_{\mathrm{ac}}$ & Self-absorption & $(1+Y)^{-3 / 5}$ & $(1+Y)^{-6 / 5}$ \\
8 & $\nu_{\mathrm{sa}}$ & Self-absorption & $(1+Y)^{-1 / 3}$ & $(1+Y)^{-5 / 6}$ \\
9 & $\nu_{\mathrm{m}}$ & Characteristic & $(1+Y)^{0}$ & $(1+Y)^{-1}$ \\
10 & $\nu_{\mathrm{sa}}$ & Self-absorption & $(1+Y)^{1}$ & $(1+Y)^{1}$ \\
11 & $\nu_{\mathrm{c}}$ & Cooling & $(1+Y)^{-2}$ & $(1+Y)^{0}$ \\
\hline
\end{tabular}

National Science Foundation under cooperative agreement by Associated universities, Inc. The Berger GRB group at Harvard is supported in part by the National Science Foundation under Grant AST-1107973. B.A.Z. acknowledges support from NSF AST-1302954. D.A.P. is supported by Hubble Fellowship grant HST-HF-51296.01-A, and by NASA through an award issued by JPL/Caltech as part of Spitzer proposal GO-90082. This research has made use of data obtained through the High Energy Astrophysics Science Archive Research Center Online Service, provided by the NASA/Goddard Space Flight Center.

\section{APPENDIX A INVERSE COMPTON CORRECTION}

The synchrotron photons produced in the GRB blastwave can Compton-scatter off the shock-accelerated relativistic electrons in the blastwave, producing a Comptonized spectrum at high energies (Blandford \& McKee 1977; Panaitescu \& Mészáros 1998; Totani 1998; Wei \& Lu 1998; Chiang \& Dermer 1999; Dermer et al. 2000a, 2000b; Panaitescu \& Kumar 2000; Sari \& Esin 2001). We compute the IC spectrum by directly integrating the synchrotron spectrum over the electron Lorentz factor distribution (back-calculated from the synchrotron spectrum) using Equation (A2) in Sari \& Esin (2001). We find that IC emission contributes negligible flux compared to synchrotron radiation and we ignore this component in our analysis. However, the IC mechanism can provide a significant source of cooling for the shockaccelerated electrons and thereby dominate the total cooling rate, even when IC emission itself is not directly observable (Sari et al. 1996; Sari \& Esin 2001; Zhang et al. 2007). This affects the synchrotron cooling frequency, as well as the selfabsorption frequency (when $\nu_{\mathrm{c}}<\nu_{\mathrm{m}}$ ). Thus IC cooling should be taken into account when computing SEDs and light curves for the synchrotron component.

\footnotetext{
${ }^{28}$ Note that this formula for $Y$ does not take the Klein-Nishina correction into account. This frequency-dependent correction is expected to be important only at high frequencies, $\nu \gtrsim 10^{18} \mathrm{~Hz}$ at $t \gtrsim 1 \mathrm{~d}$ (Fan \& Piran 2006; Zhang et al. 2007). Upon detailed investigation, we find that a consequence of this effect is to reduce the overall energy required to match the light curves by up to $25 \%$. The uncertainties arising from model selection as well as due to correlations between parameters are usually also of this order and we therefore do not consider this effect further in this work.
}

The importance of IC cooling is determined by the Compton $y$-parameter, ${ }^{28}$

$$
Y=\frac{-1+\sqrt{1+4 \eta \epsilon_{\mathrm{e}} / \epsilon_{B}}}{2},
$$

where $\eta$ is the fraction of energy that has been radiated away due to synchrotron and IC radiation, such that $\eta=1$ during fast cooling and $\eta=\left(\nu_{\mathrm{c}} / \nu_{\mathrm{m}}\right)^{-(p-2) / 2}$ during slow cooling (Sari \& Esin 2001). Writing $\nu_{\mathrm{c}}=\nu_{\mathrm{c}}^{\prime}(1+Y)^{-2}$, where $\nu_{\mathrm{c}}^{\prime}$ is the cooling frequency of the synchrotron SED not corrected for IC cooling, we have

$$
\begin{aligned}
\eta & =\left[\frac{\nu_{\mathrm{c}}^{\prime}(1+Y)^{-2}}{\nu_{\mathrm{m}}}\right]^{-(p-2) / 2}=\left(\frac{\nu_{\mathrm{c}}^{\prime}}{\nu_{\mathrm{m}}}\right)^{-(p-2) / 2}(1+Y)^{p-2} \\
& =H\left[\frac{1+\sqrt{1+4 \eta \epsilon_{\mathrm{e}} / \epsilon_{B}}}{2}\right]^{p-2},
\end{aligned}
$$

where $H=\left(\nu_{\mathrm{c}}^{\prime} / \nu_{\mathrm{m}}\right)^{-(p-2) / 2}$ is independent of $\eta$. We therefore obtain the following implicit equation for $\eta$,

$$
f(\eta) \equiv \eta-H\left[\frac{1+\sqrt{1+4 \eta \epsilon_{\mathrm{e}} / \epsilon_{B}}}{2}\right]^{p-2}=0,
$$

which can be solved numerically using (for instance) the Newton-Raphson method. Finally, the effect of IC cooling can be accounted for by scaling the spectral break frequencies and flux densities of the synchrotron spectrum by the appropriate powers of $1+Y$ (Granot \& Sari 2002). For convenience, we summarize these scaling relations in Table 10.

\section{APPENDIX B \\ A WIND MODEL FOR GRB 120326A}

In Sections 5.1 we discussed the ISM model for GRB 120326A. We now consider the possibility of a wind-like circumburst environment.

The best-fit model in a wind environment requires $p \approx 2.52$, $\epsilon_{\mathrm{e}} \approx 4.9 \times 10^{-2}, \epsilon_{B} \approx 1.0 \times 10^{-2}, A_{*} \approx 7.0 \times 10^{-2}, E_{\mathrm{K}, \text { iso }} \approx$ $3.6 \times 10^{54} \quad \mathrm{erg}, \quad t_{\mathrm{jet}} \approx 19 \quad \mathrm{~d}, \quad A_{\mathrm{V}} \approx 0.46 \mathrm{mag}$, and $F_{\nu, \text { host, } \mathrm{r}^{\prime}} \approx 1.6 \mu \mathrm{Jy}$. This model transitions from fast cooling to slow cooling at $3.5 \times 10^{-2} \mathrm{~d}$. The spectral break frequencies at $1 \mathrm{~d}$ are located at $\nu_{\mathrm{a}} \approx 6.8 \times 10^{8} \mathrm{~Hz}, \nu_{\mathrm{m}} \approx 2.2 \times 10^{14} \mathrm{~Hz}$, and $\nu_{\mathrm{c}} \approx 4.2 \times 10^{15} \mathrm{~Hz}$. The peak of the $\operatorname{spectrum}\left(f_{\nu}\right)$ is at $\nu_{\mathrm{m}}$, with a flux density of $\approx 23 \mathrm{mJy}$.

From our MCMC simulations, we find $p=2.52 \pm 0.02$, $E_{\mathrm{K} \text {,iso }}=\left(3.1_{-0.5}^{+0.9}\right) \times 10^{54} \mathrm{erg}, \quad A_{*}=\left(4.8_{-2.5}^{+3.3}\right) \times 10^{-2}, \quad \epsilon_{\mathrm{e}}=$ $\left(4.1_{-1.2}^{+1.3}\right) \times 10^{-2}, \epsilon_{B}=\left(2.8_{-2.1}^{+9.4}\right) \times 10^{-2}$, and $t_{\text {jet }}=18.7_{-2.9}^{3.1} \mathrm{~d}$. Using the relation $\theta_{\mathrm{jet}}=0.17\left(2 \frac{t_{\mathrm{jet}} * A_{*}}{(1+z) E_{\mathrm{K}, \mathrm{is}, 52}}\right)^{1 / 4}$ for the jet opening angle (Chevalier \& Li 2000), and the distributions of $E_{\mathrm{K} \text {,iso }}, n_{0}$, and $t_{\text {jet }}$ from our MCMC simulations (Figure 27), we find $\theta_{\text {jet }}=2: 1_{-0.3}^{+0.2}$. Applying the beaming correction, $E_{\gamma}=$ $E_{\gamma \text {,iso }}\left(1-\cos \theta_{\text {jet }}\right)$, we find $E_{\gamma}=(2.1 \pm 0.3) \times 10^{49} \mathrm{erg}$. The beaming-corrected kinetic energy is much larger, $E_{\mathrm{K}}=$ $\left(2.0_{-0.6}^{+1.0}\right) \times 10^{51} \mathrm{erg}$. In this model, $\nu_{\mathrm{a}}$ falls below $10^{10} \mathrm{~Hz}$ at $1.7 \times 10^{-2} \mathrm{~d}$ and is therefore not probed by any of the radio data. Consequently, the model exhibits a degeneracy in its parameters due to to the unknown value of $\nu_{\mathrm{a}}$ (Figure 28). This 

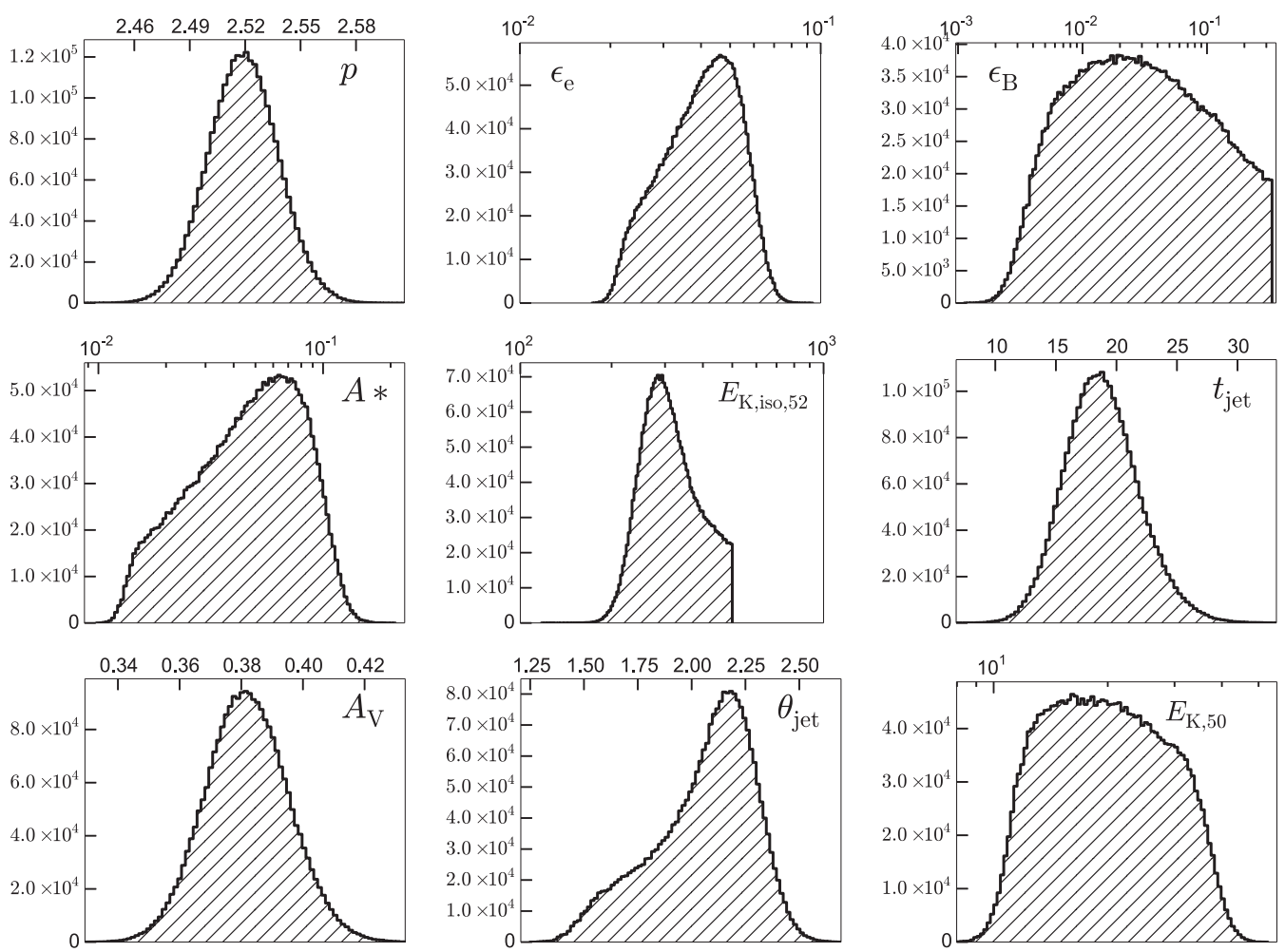

Figure 27. Posterior probability density functions for the physical parameters for GRB 120326A in the wind model from MCMC simulations. We have restricted

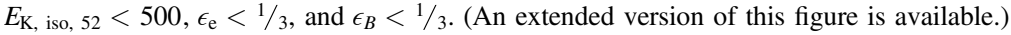

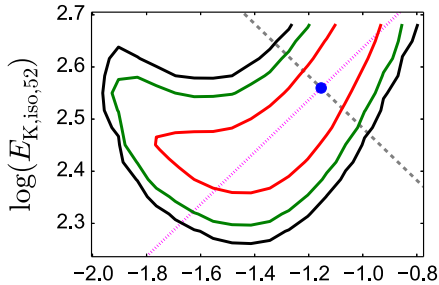

$\log (A *)$

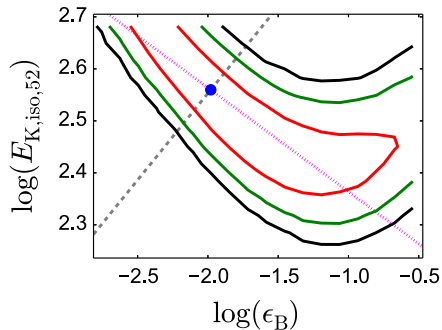

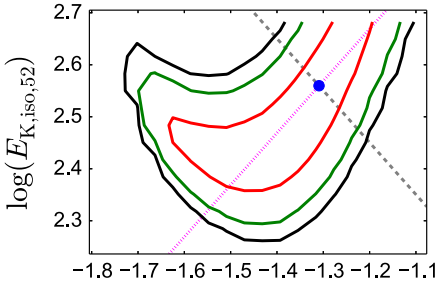

$\log \left(\epsilon_{\mathrm{e}}\right)$

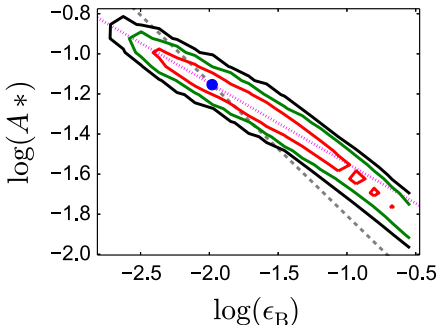

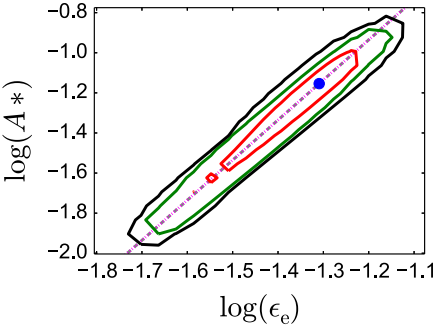

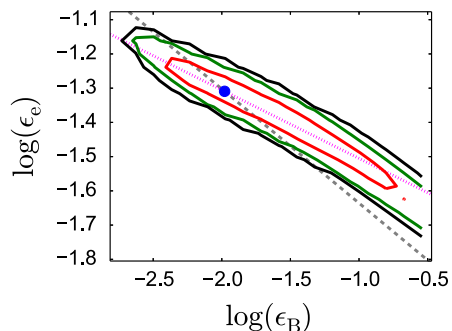

Figure 28. $1 \sigma$ (red), $2 \sigma$ (green), and $3 \sigma$ (black) contours for correlations between the physical parameters, $E_{\mathrm{K}}, A_{*}, \epsilon_{\mathrm{e}}$, and $\epsilon_{B}$ for GRB $120326 \mathrm{~A}$, in the wind model

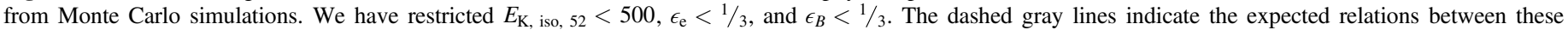
parameters when $\nu_{\mathrm{a}}$ is not fully constrained: $E_{\mathrm{K}, \text { iso }} \propto A_{*}^{-1 / 2}, E_{\mathrm{K}, \text { iso }} \propto \epsilon_{\mathrm{e}}^{-1}, A_{*} \propto \epsilon_{\mathrm{e}}^{2}, E_{\mathrm{K} \text {,iso }} \propto \epsilon_{B}^{1 / 3}, A_{*} \propto \epsilon_{B}^{-2 / 3}$, and $\epsilon_{\mathrm{e}} \propto \epsilon_{B}^{-1 / 3}$, normalized to pass through the highest-likelihood point (blue dot), while the dotted magenta lines indicate expected relations for changes the value of $\nu_{\mathrm{c}}: E_{\mathrm{K} \text {,iso }} \propto A_{*}^{1 / 2}, E_{\mathrm{K} \text {,iso }} \propto \epsilon_{\mathrm{e}}, A_{*} \propto \epsilon_{\mathrm{e}}^{2}$, $E_{\mathrm{K}, \text { iso }} \propto \epsilon_{B}^{-1 / 5}, A_{*} \propto \epsilon_{B}^{-2 / 5}$, and $\epsilon_{\mathrm{e}} \propto \epsilon_{B}^{-1 / 5}$. $\nu_{\mathrm{a}}$ falls below the radio band before any radio observations took place, and is therefore unconstrained. $\nu_{\mathrm{c}}$ lies between the optical and X-ray bands and is better constrained; the correlations between the parameters along the lines of varying values of $\nu_{\mathrm{c}}$ are likely indicative of the strong changes (over two orders of magnitude) in the Compton $y$-parameter along these curves. See the online version of this figure for additional plots of correlations

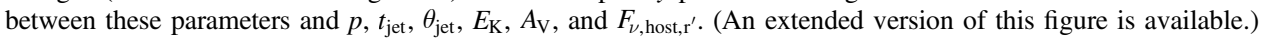

high value of $E_{\mathrm{K}}$ also implies a low radiative efficiency, $\eta_{\text {rad }} \equiv E_{\gamma} /\left(E_{\mathrm{K}}+E_{\gamma}\right) \approx 1 \%$.

We now investigate the effect of energy injection in causing an X-ray/UV/optical re-brightening. The X-ray light curve during the re-brightening is located above the cooling frequency. In the wind model, the flux density above $\nu_{\mathrm{c}}$ is $F_{\nu>\nu_{\mathrm{c}}} \propto E_{\mathrm{K} \text {,iso }}^{(2+p) / 4} t^{(2-3 p) / 4}$ (Granot \& Sari 2002). For $p=2.5$, this reduces to $F_{\nu>\nu_{\mathrm{c}}} \propto E_{\mathrm{K} \text {,iso }}^{1.13} t^{-1.38}$. During energy injection, $E \propto t^{m}$, such that $F_{\nu>\nu_{\mathrm{c}}} \propto t^{1.13 m-1.38}$. The steep rise $\left(\alpha_{1}=0.85 \pm 0.19 ;\right.$ Section 3.1) requires $m=2.0 \pm 0.2$. 

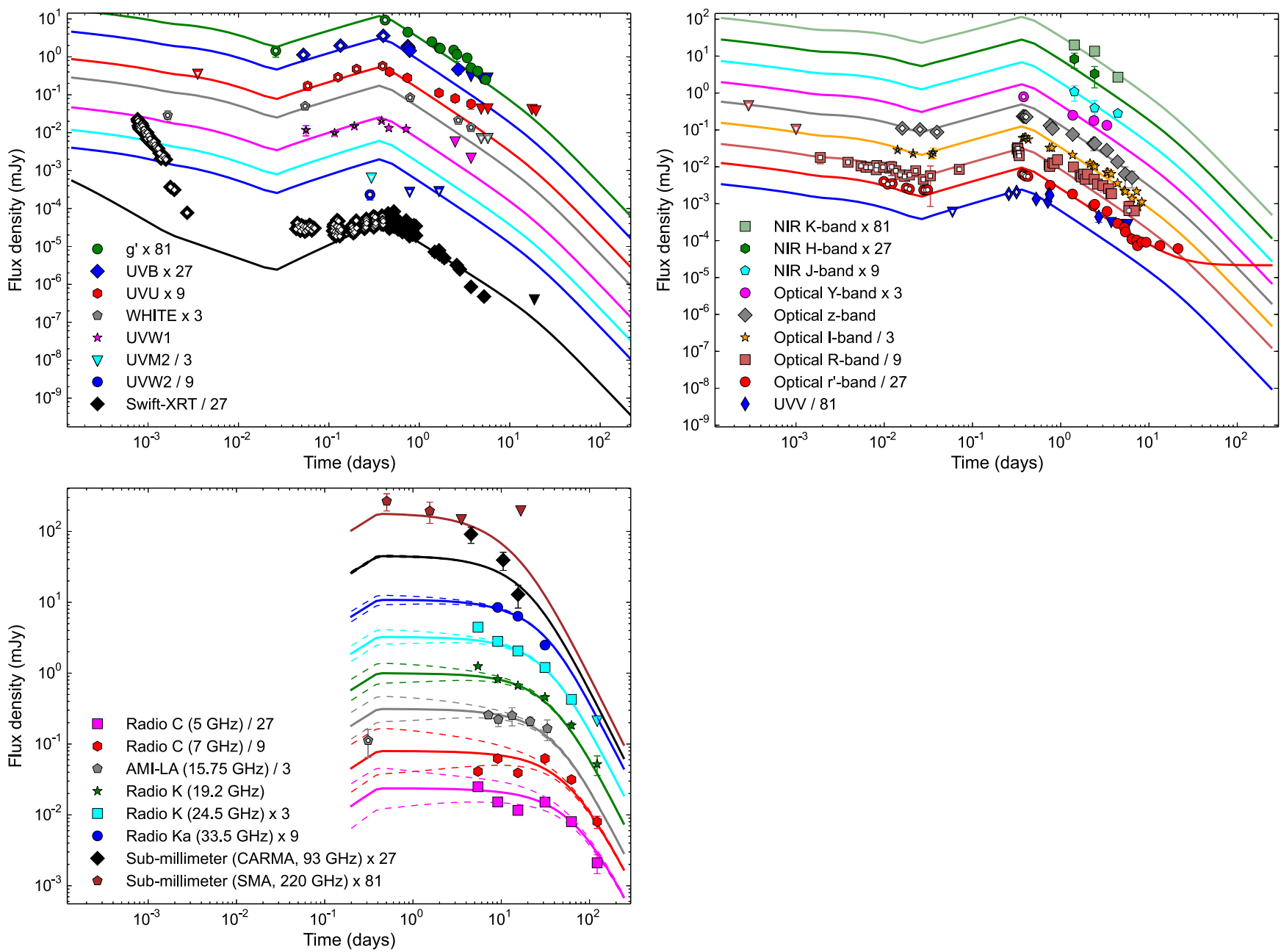

Figure 29. X-ray, UV (top left), optical (top right), and radio (bottom left) light curves of GRB 120326A in the wind scenario, with the full afterglow model (solid lines), including energy injection before $0.4 \mathrm{~d}$.
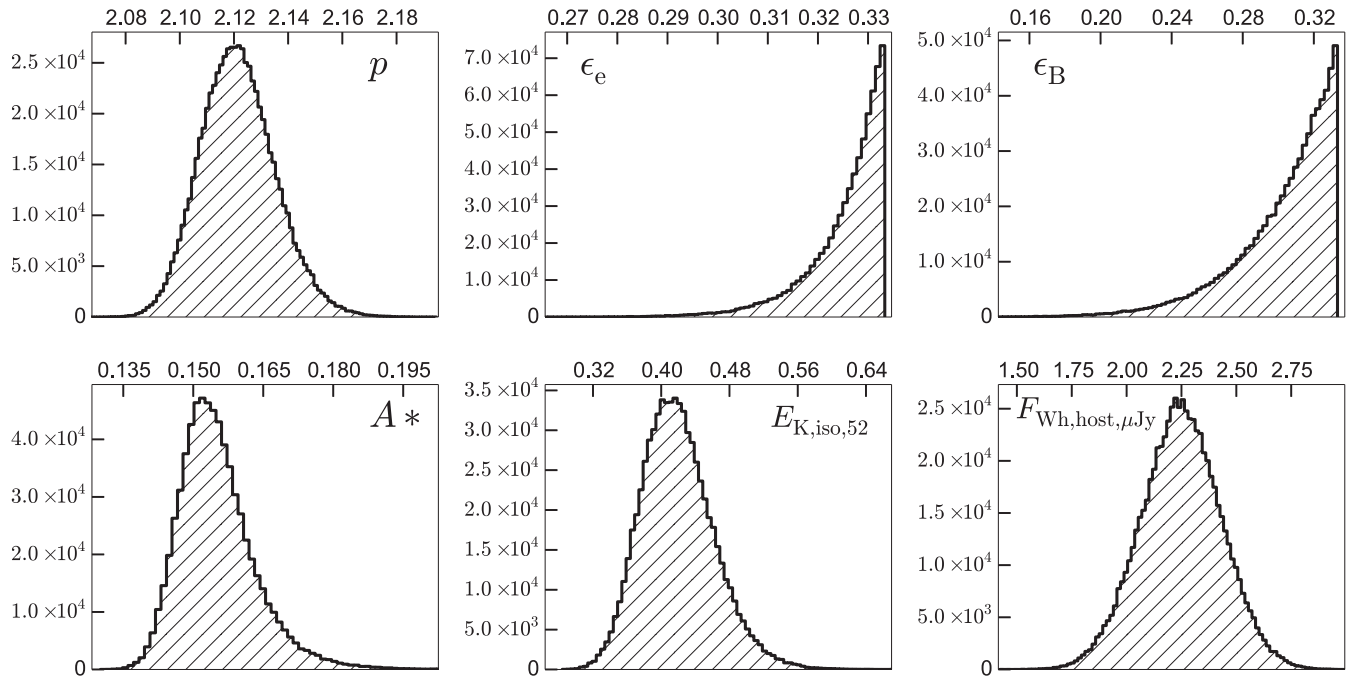

Figure 30. Posterior probability density functions for the physical parameters for GRB 100418A in a wind environment from MCMC simulations. We have restricted $E_{\mathrm{K}, \text { iso, } 52}<500, \epsilon_{\mathrm{e}}<1 / 3$, and $\epsilon_{B}<1 / 3$. The last panel corresponds to the flux density of the host galaxy in the Swift/White band. 

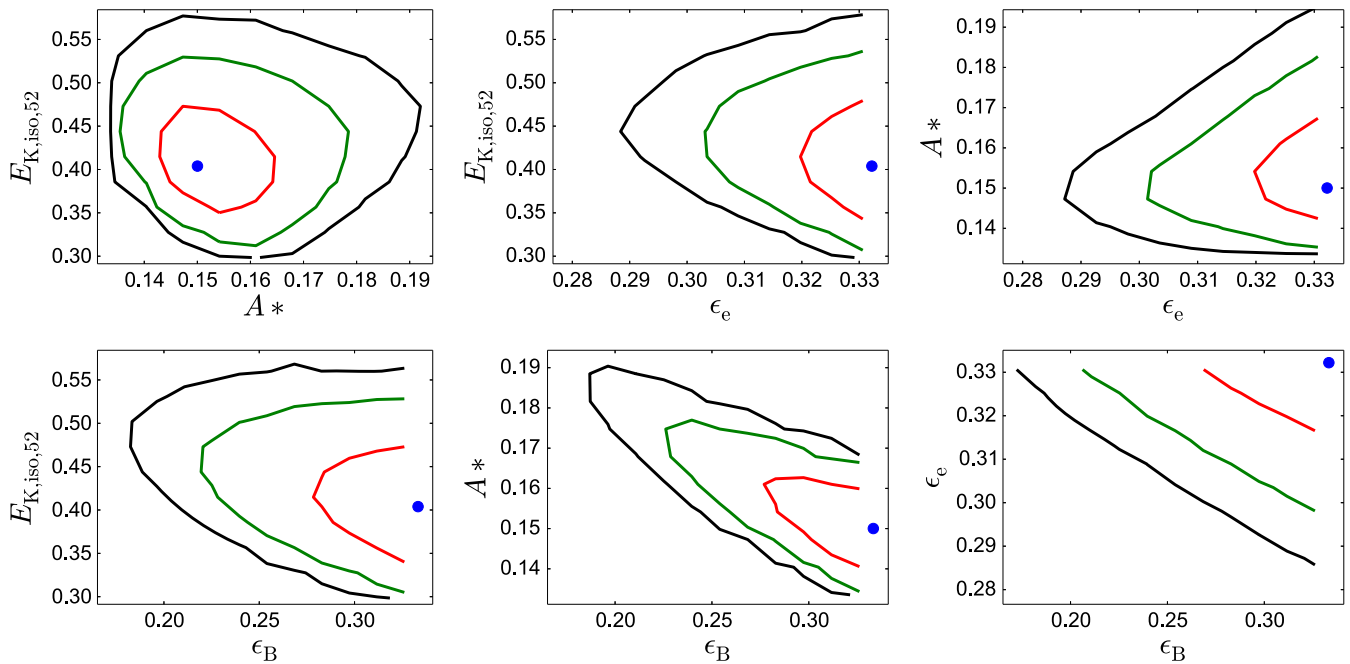

Figure 31. $1 \sigma$ (red), $2 \sigma$ (green), and $3 \sigma$ (black) contours for correlations between the physical parameters, $E_{\mathrm{K}, \text { iso }}, n_{0}, \epsilon_{\mathrm{e}}$, and $\epsilon_{B}$ for GRB 100418A, in the wind model from Monte Carlo simulations. We have restricted $E_{\mathrm{K}, \text { iso,52 }}<500, \epsilon_{\mathrm{e}}<1 / 3$, and $\epsilon_{B}<1 / 3$. The highest-likelihood model is marked with a blue dot. See the on line

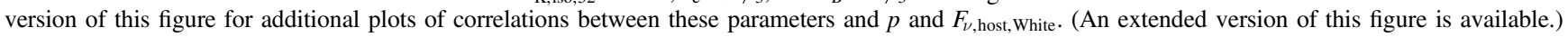
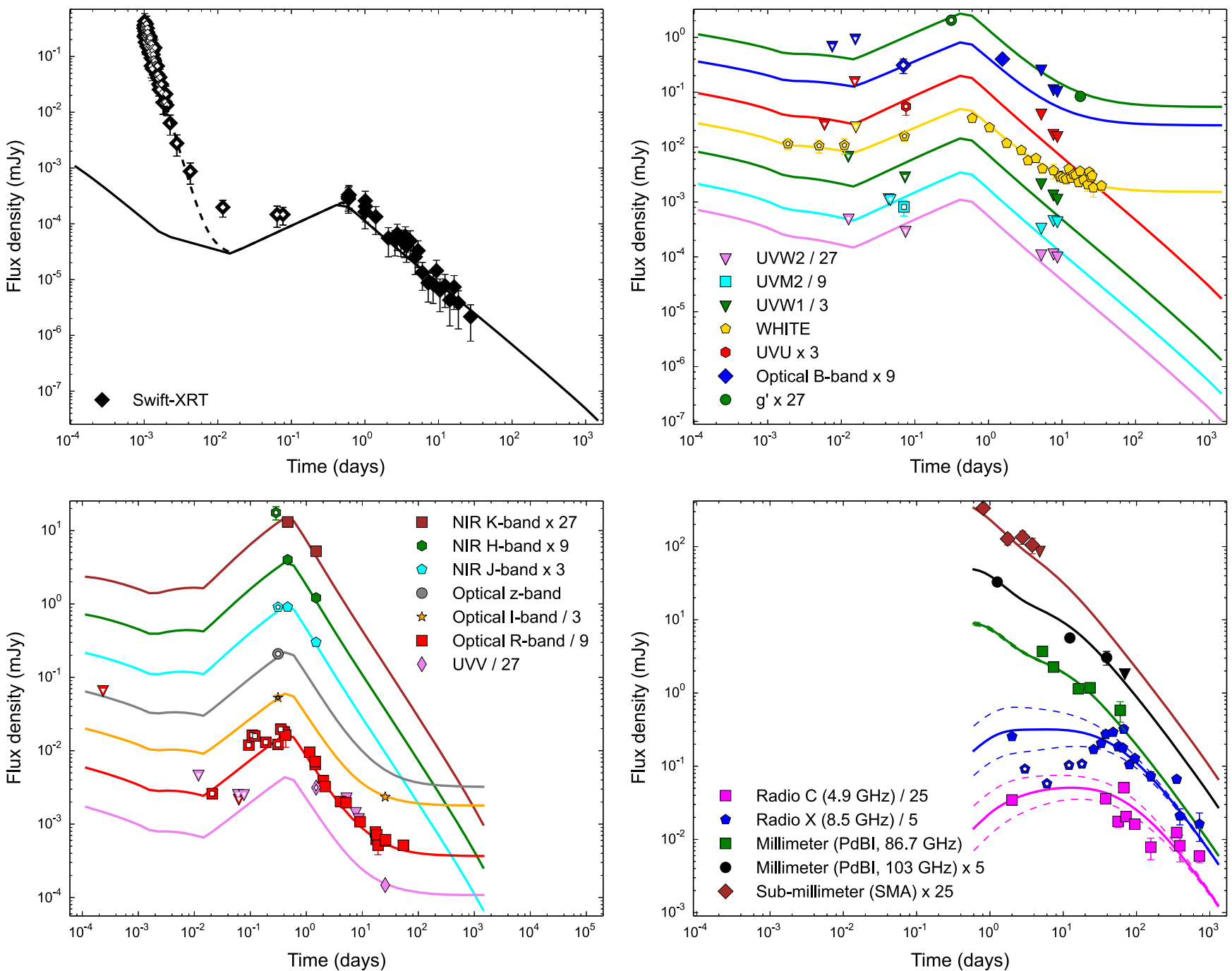

Figure 32. X-ray (top left), UV (top right), optical (bottom left), and radio (bottom right) light curves of GRB 100418A in the wind scenario, with the full afterglow model (solid lines), including energy injection before $0.5 \mathrm{~d}$. 

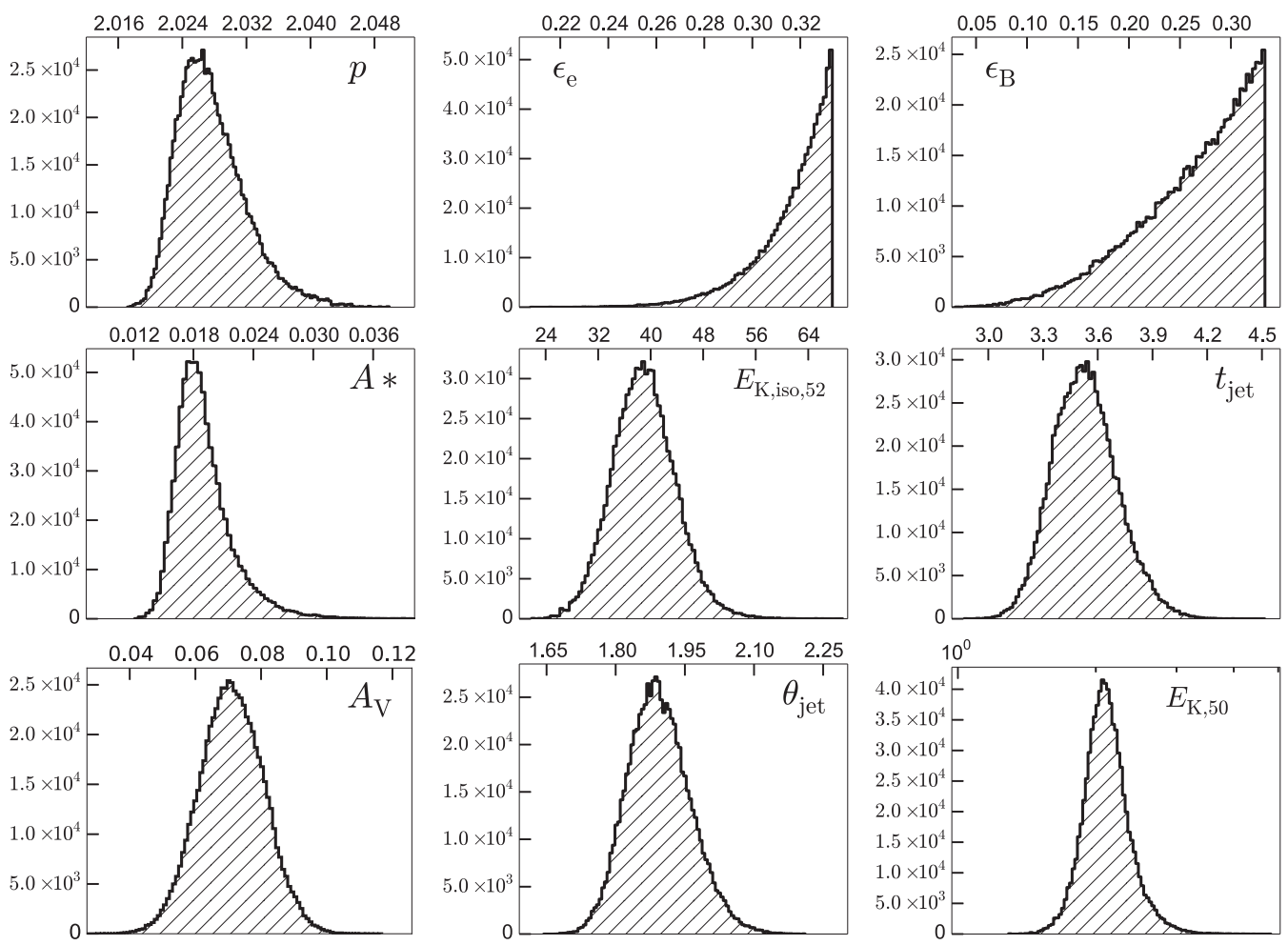

Figure 33. Posterior probability density functions for the physical parameters for GRB 100901A in a wind environment from MCMC simulations. We have restricted

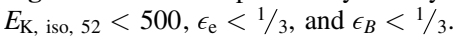
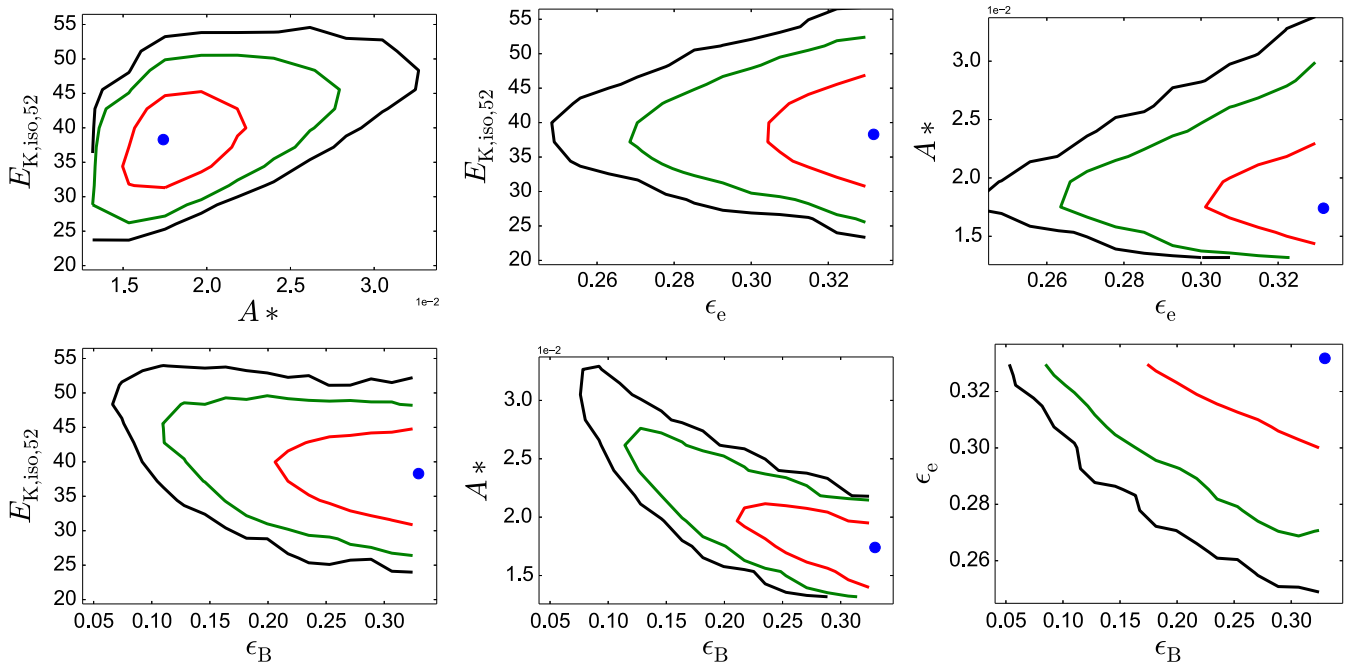

Figure 34. $1 \sigma$ (red), $2 \sigma$ (green), and $3 \sigma$ (black) contours for correlations between the physical parameters, $E_{\mathrm{K}, \text { iso }}, n_{0}, \epsilon_{\mathrm{e}}$, and $\epsilon_{B}$ for GRB $100901 \mathrm{~A}$, in the wind model from Monte Carlo simulations. We have restricted $E_{\mathrm{K}}$, iso, 52 $<500, \epsilon_{\mathrm{e}}<1 / 3$, and $\epsilon_{B}<1 / 3$. The highest-likelihood model is marked with a blue dot. See the online version of this figure for additional plots of correlations between these parameters and $p, A_{\mathrm{V}}, t_{\mathrm{jet}}, \theta_{\mathrm{jet}}$, and $E_{\mathrm{K}}$. (An extended version of this figure is available.)

However, $0 \leqslant m<1$ is bounded (Section 5.2.4), which implies that in this model, energy injection due to a distribution of ejecta energy to lower Lorentz factors can not cause the $\mathrm{X}$-ray flux to rise with time.

Relaxing the requirement $m<1$, our best solution for the multi-wavelength re-brightening for an energy injection model requires two periods of energy injection (Figure 29). In the first episode between $1.7 \times 10^{-3} \mathrm{~d}$ and $2.5 \times 10^{-2} \mathrm{~d}, E_{\mathrm{K} \text {,iso }}$ increases as $t^{0.5}$ growing by a factor of 3.9 from $1.6 \times 10^{51}$ erg to $6.2 \times 10^{51} \mathrm{erg}$. In the second episode, $E_{\mathrm{K} \text {,iso }} \propto t^{2.3}$ from $2.5 \times 10^{-2} \mathrm{~d}$ to $0.4 \mathrm{~d}$, further increasing by a factor of over 2000 to its final value of $E_{\mathrm{K} \text {,iso }} \approx 3.6 \times 10^{54} \mathrm{erg}$ in this period. The resulting light curves, which are optimized to match the UV and optical re-brightening, cannot reproduce the X-ray light curve prior to the re-brightening (a shallower rise in the X-rays in the ISM model was achieved by placing $\nu_{\mathrm{m}}$ between the optical and X-rays). Due to the extremely large injected energy coupled with the fact that the steep rise 

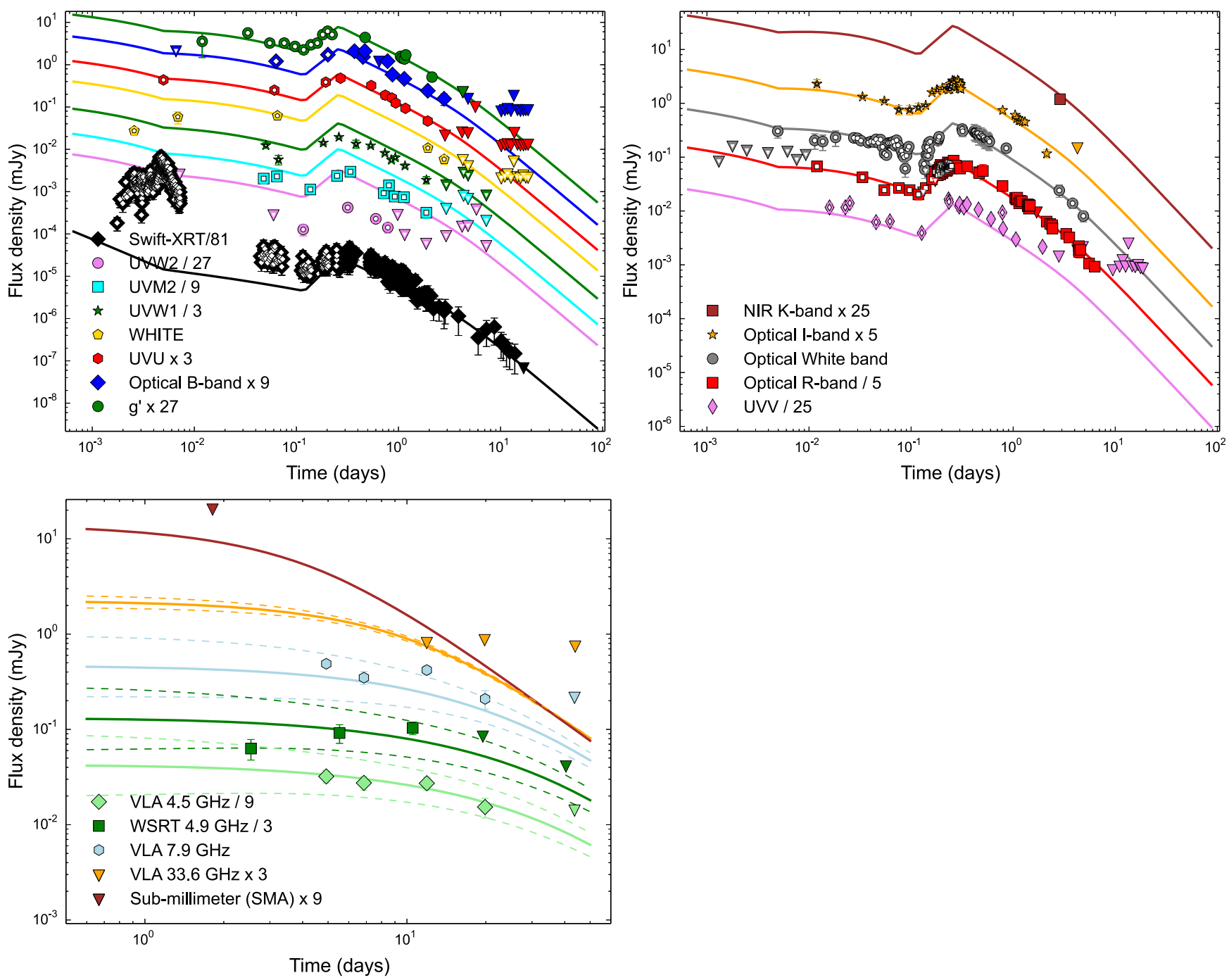

Figure 35. X-ray and UV (top left), optical (top right), and radio (bottom left) light curves of GRB 100901A in the wind scenario, with the full afterglow model (solid lines), including energy injection before $0.26 \mathrm{~d}$.

in the optical violates the bounds on $m$, the wind model is a less attractive solution for the multi-wavelength afterglow of GRB 120326A.

\section{APPENDIX C \\ A WIND MODEL FOR GRB 100418A}

We apply our MCMC analysis described in Section 6.1.2 to explore afterglow models with a wind-like circumburst environment for GRB 100418A. The parameters of the highest-likelihood model are $p \approx 2.1, \epsilon_{\mathrm{e}} \approx 0.33, \epsilon_{B} \approx 0.33$, $A_{*} \approx 0.15, E_{\mathrm{K} \text {,iso }} \approx 4.0 \times 10^{51} \mathrm{erg}$, and $F_{\nu, \text { host, White }} \approx 2.3 \mu \mathrm{Jy}$, with negligible extinction. This model transitions from fast to slow cooling at $1.5 \mathrm{~d}$. The spectral break frequencies at $1 \mathrm{~d}$ are located at $\nu_{\mathrm{ac}} \approx 5.8 \mathrm{GHz}, \nu_{\mathrm{sa}} \approx 32 \mathrm{GHz}, \nu_{\mathrm{c}} \approx 6.4 \times 10^{11} \mathrm{~Hz}$, and $\nu_{\mathrm{m}} \approx 3.6 \times 10^{12} \mathrm{~Hz}$ at $1 \mathrm{~d}$, with $F_{\max } \approx 50 \mathrm{mJy}$ at $\nu_{\mathrm{c}}$ at $1 \mathrm{~d}$ and a Compton $y$-parameter of 0.6.

Like in the ISM model, the optical and X-ray bands are located above both $\nu_{\mathrm{m}}$ and $\nu_{\mathrm{c}}$. This model does not require a jet break, and we find $t_{\text {jet }} \gtrsim 140 \mathrm{~d}$. Thus we cannot constrain $\theta_{\text {jet }}$ in this model, nor correct $E_{\gamma, \text { iso }}$ or $E_{\mathrm{K} \text {,iso }}$ for beaming.
The summary statistics from our MCMC simulations are $p=2.12 \pm 0.01, \quad E_{\mathrm{K}, \text { iso }}=(4.2 \pm 0.4) \times 10^{51} \mathrm{erg}, \quad A_{*}=$ $0.16_{-0.006}^{+0.008}, \quad \epsilon_{\mathrm{e}}=0.328_{-0.009}^{+0.004}$, and $\epsilon_{B}=0.31_{-0.02}^{+0.03}$. We plot histograms of the posterior density functions for these parameters in Figure 30 and present contours of the joint posterior density for the physical parameters $A_{*}, E_{\mathrm{K} \text {,iso }}, \epsilon_{\mathrm{e}}$, and $\epsilon_{B}$ in Figure 31.

It is challenging to fit both the X-ray and optical light curves before the bump together in the energy injection scenario under the wind model. Like for the wind model for GRB 120326A (Appendix B), we find that the optical light curves before the peak require a steeper injection rate than allowed by a distribution of Lorentz factors in the ejecta. In particular, our best energy injection model that matches the optical well but slightly under-predicts the X-ray data before $0.5 \mathrm{~d}$ (Figure 32 ), requires $E \propto t^{0.7}$ between $1.8 \times 10^{-3} \mathrm{~d}$ and $1.5 \times 10^{-2} \mathrm{~d}$, steepening to $E \propto t^{1.65}$ between $1.5 \times 10^{-2} \mathrm{~d}$ and $0.5 \mathrm{~d}$. In this model, the blastwave kinetic energy increases by a factor of 4.4 between $1.8 \times 10^{-3} \mathrm{~d}$ and $1.5 \times 10^{-2} \mathrm{~d}$, and another factor of $\approx 325$ between $1.5 \times 10^{-2} \mathrm{~d}$ and $0.5 \mathrm{~d}$, for an overall increase 

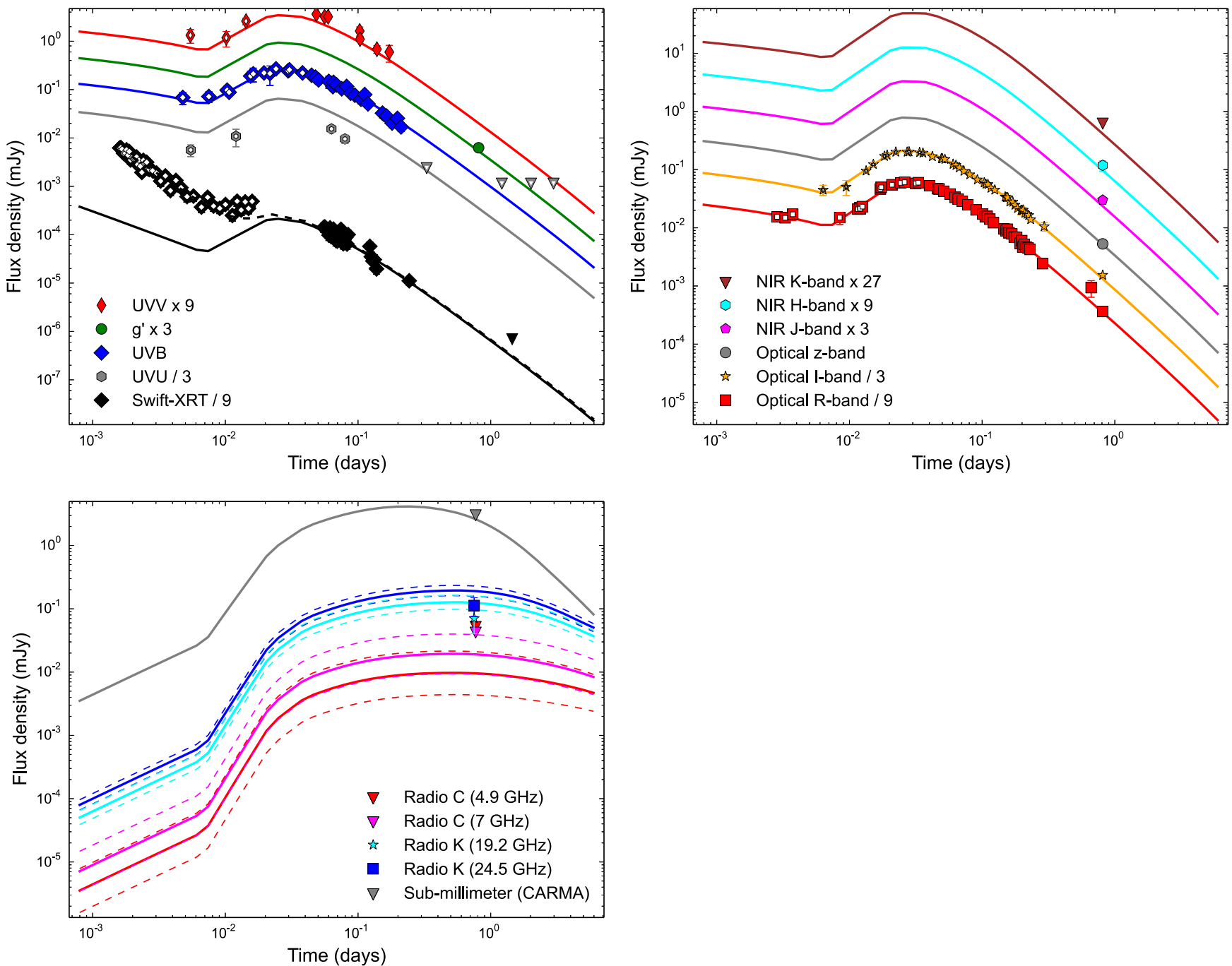

Figure 36. Similar to Figure 20, but for a wind-like circumburst environment. The light curves before $0.04 \mathrm{~d}$ are based on the same energy injection model described in Section 6.3.3 as applied to the case of the wind medium. See Appendix E for a discussion.
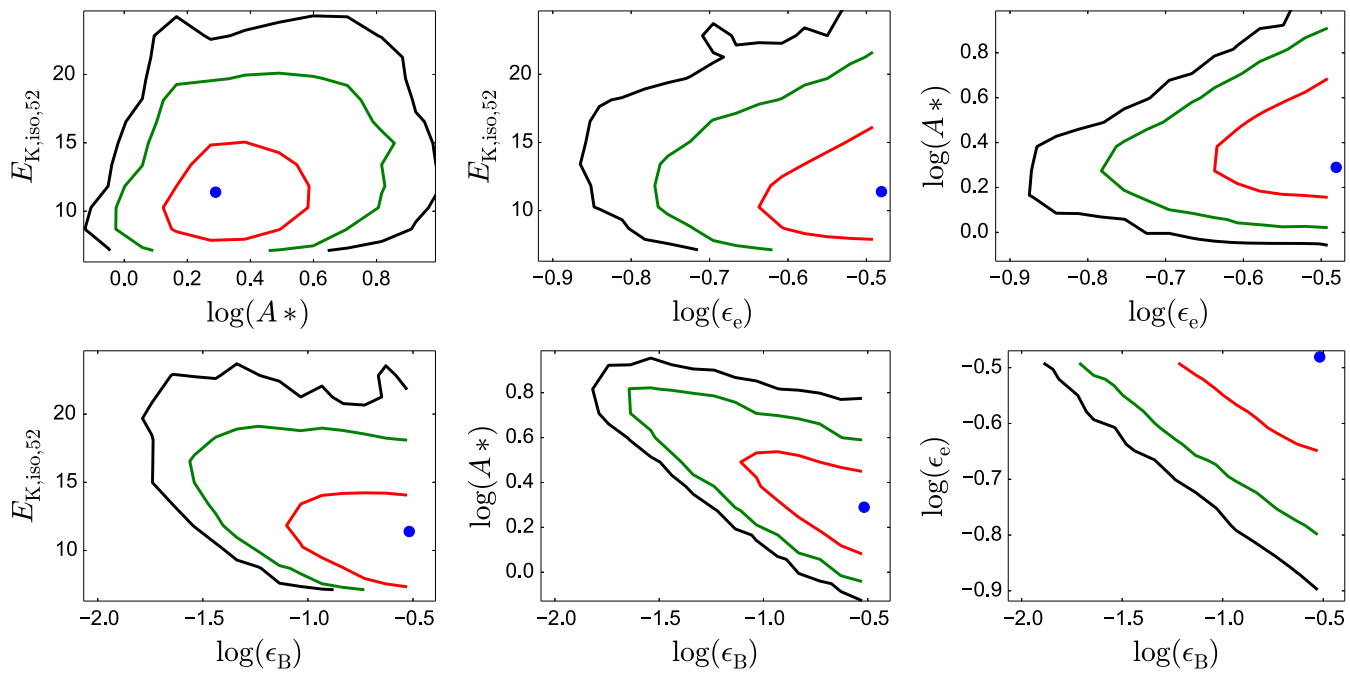

Figure 37. $1 \sigma$ (red), $2 \sigma$ (green), and $3 \sigma$ (black) contours for correlations between the physical parameters, $E_{\mathrm{K}, \text { iso }}, n_{0}, \epsilon_{\mathrm{e}}$, and $\epsilon_{B}$ for GRB $120404 \mathrm{~A}$, in the wind model from Monte Carlo simulations. We have restricted $\epsilon_{\mathrm{e}}<1 / 3$ and $\epsilon_{B}<1 / 3$. The highest-likelihood model is marked with a blue dot. See the online version of this figure for additional plots of correlations between these parameters and $p, t_{\mathrm{jet}}, \theta_{\mathrm{jet}}, E_{\mathrm{K}}$, and $A_{\mathrm{V}}$. (An extended version of this figure is available.) 

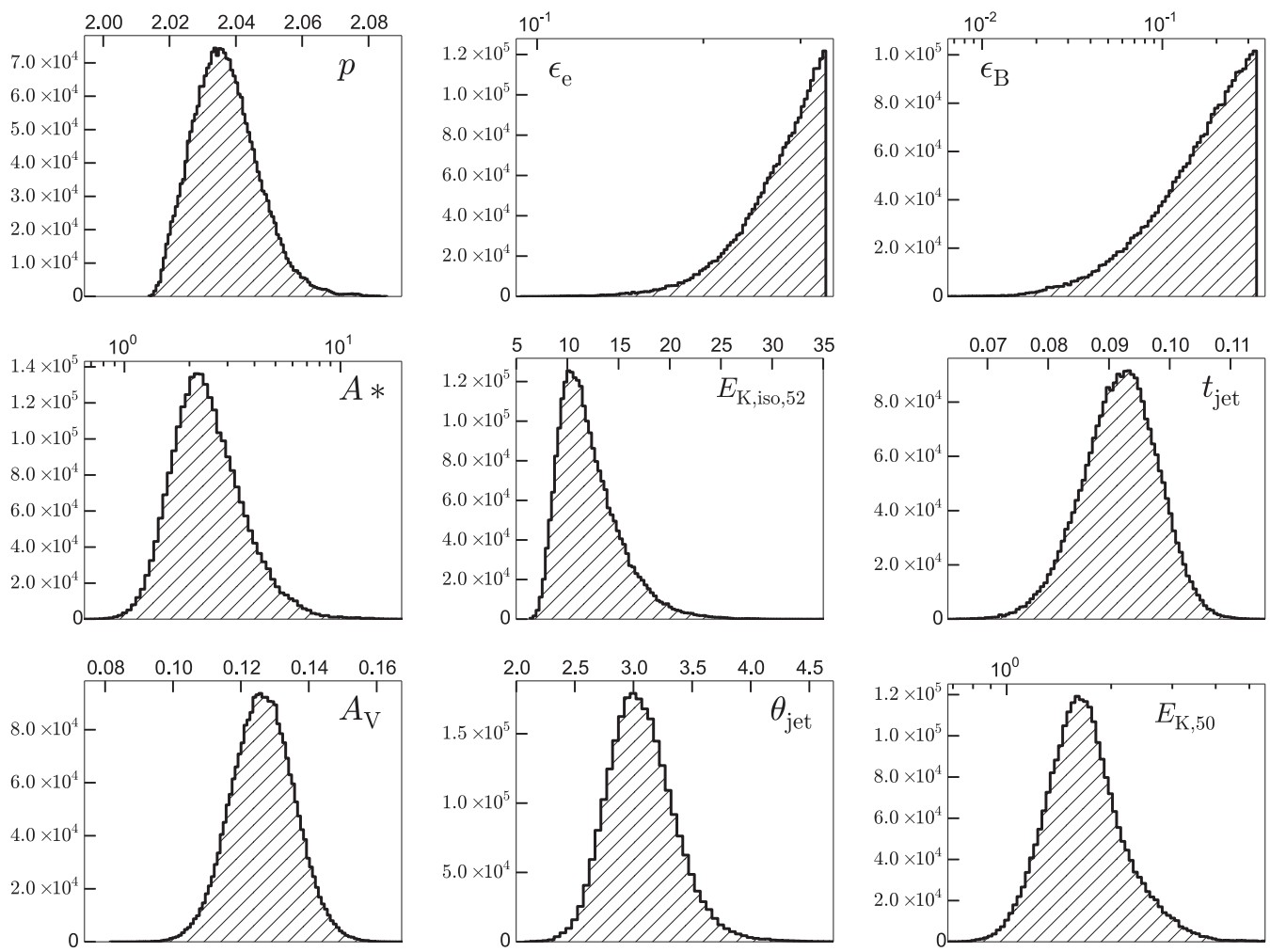

Figure 38. Posterior probability density functions for the physical parameters for GRB 120404A in a wind environment from MCMC simulations. We have restricted $\epsilon_{\mathrm{e}}<1 / 3$ and $\epsilon_{B}<1 / 3$.

by a factor of 1440. Due to the large injection of energy required to account for the optical re-brightening in this model, we do not consider the wind environment to be a likely explanation for the multi-wavelength afterglow of GRB 100418A.

\section{APPENDIX D \\ A WIND MODEL FOR GRB 100901A}

We apply our MCMC analysis described in Section 6.2.2 to explore afterglow models with a wind-like circumburst environment for GRB 100901A. The parameters of the highest-likelihood model are $p \approx 2.02, \epsilon_{\mathrm{e}} \approx 0.33, \epsilon_{B} \approx 0.33, A_{*} \approx 1.7 \times 10^{-2}$, $E_{\mathrm{K}, \text { iso }} \approx 3.8 \times 10^{53}$, and $A_{\mathrm{V}} \lesssim 0.1$ mag. This model transitions from fast to slow cooling at $5.4 \times 10^{-2} \mathrm{~d}$. The spectral break frequencies at $1 \mathrm{~d}$ are located at $\nu_{\mathrm{a}} \approx 3.1 \times 10^{8} \mathrm{~Hz}$, $\nu_{\mathrm{m}} \approx 1.8 \times 10^{12} \mathrm{~Hz}$, and $\nu_{\mathrm{c}} \approx 1.7 \times 10^{15} \mathrm{~Hz}$, with $F_{\max } \approx 3.8$ mJy at $1 \mathrm{~d}$, and a Compton $y$-parameter of 0.6. Like in the ISM model, $\nu_{\mathrm{c}}$ is located between the optical and X-ray bands. Since the light curves in a wind environment decline faster than in the ISM case, the jet break in this model is later $\left(t_{\text {jet }} \approx 3.5 \mathrm{~d}\right)$ compared to the ISM model $\left(t_{\text {jet }} \approx 1 \mathrm{~d}\right)$. The jet opening angle is $\theta_{\text {jet }} \approx 1.9$, resulting in a beaming-corrected kinetic energy of $2.0 \times 10^{50} \mathrm{erg}$.

The summary statistics from our MCMC simulations are $p=2.027_{-0.003}^{+0.005}, E_{\mathrm{K}, \text { iso }}=(3.9 \pm 0.5) \times 10^{53} \mathrm{erg}, A_{*}=1.9_{-0.2}^{+0.3} \times$ $10^{-2}, \quad \epsilon_{\mathrm{e}}=0.32_{-0.02}^{+0.01}, \quad \epsilon_{B}=0.27_{-0.07}^{+0.04}, \quad A_{\mathrm{V}} \lesssim 0.1 \mathrm{mag}, \quad t_{\mathrm{jet}}=$ $3.5 \pm 0.2 \mathrm{~d}, \theta_{\mathrm{jet}}=1.9 \pm 0.1, E_{\mathrm{K}}=(2.1 \pm 0.2) \times 10^{50} \mathrm{erg}$, and $E_{\gamma}=4.4 \pm 0.7 \times 10^{49} \mathrm{erg}$. We plot histograms of the posterior density functions for these parameters in Figure 33 and present contours of the joint posterior density for the physical parameters $A_{*}, E_{\mathrm{K}, \text { iso }}, \epsilon_{\mathrm{e}}$, and $\epsilon_{B}$ in Figure 34.
Like for GRBs 100418A and 120326A, it is challenging to fit both the X-ray and optical light curves before the bump together in the energy injection scenario under the wind model. Like for the wind model for GRB 120326A (Appendix B) and GRB 100418A (Appendix C), the optical light curves before the peak require a steeper injection rate than allowed by a distribution of Lorentz factors in the ejecta. In particular, our best energy injection model that matches the optical well but slightly under-predicts the X-ray data before $0.15 \mathrm{~d}$ (Figure 35), requires $E \propto t^{0.7}$ between $5 \times 10^{-3} \mathrm{~d}$ and $0.12 \mathrm{~d}$, steepening to $E \propto t^{3.5}$ between $0.12 \mathrm{~d}$ and $0.26 \mathrm{~d}$. In this model, the blastwave kinetic energy increases by a factor of 9 between $5 \times 10^{-3} \mathrm{~d}$ and $0.12 \mathrm{~d}$, and another factor of 15 between $0.12 \mathrm{~d}$ and $0.26 \mathrm{~d}$, for an overall increase by a factor of $\approx 140$. Due to the discrepancy in the X-rays, the wind model may be considered a marginally viable model for GRB 100901A.

\section{APPENDIX E \\ A WIND MODEL FOR GRB 120404A}

We apply the methods described in Section 6.3.2 to explore afterglow models with a wind-like circumburst environment for GRB 120404A. The parameters of our highest-likelihood model are $p \approx 2.03, \epsilon_{\mathrm{e}} \approx 0.33, \epsilon_{B} \approx 0.30, \quad A_{*} \approx 1.9$, $E_{\mathrm{K} \text {,iso }} \approx 1.1 \times 10^{53} \mathrm{erg}, t_{\text {jet }} \approx 8.9 \times 10^{-2} \mathrm{~d}$, and $A_{\mathrm{V}} \approx 0.12$. This model also remains in spectrum 4 (Figure 17) for the duration of the observations, with the ordering $\nu_{\mathrm{c}}<$ $\nu_{\mathrm{a}}<\nu_{\mathrm{m}}$. The spectral break frequencies are located at $\nu_{\mathrm{ac}} \approx$ $2.4 \times 10^{10} \mathrm{~Hz}, \nu_{\mathrm{sa}} \approx 7.9 \times 10^{11} \mathrm{~Hz}, \nu_{\mathrm{m}} \approx 4.0 \times 10^{13} \mathrm{~Hz}$, and $\nu_{\mathrm{c}} \approx 1.5 \times 10^{10} \mathrm{~Hz}$, at $0.1 \mathrm{~d}$ with $F_{\max }=F_{\nu, \mathrm{sa}} \approx 27 \mathrm{mJy}$. The Compton $y$-parameter is 0.6 .

The summary statistics (median and $68 \%$ credible intervals) of the posterior density for these parameters are $p=$ 
$2.04 \pm 0.01, \epsilon_{\mathrm{e}}=0.28_{-0.05}^{+0.03}, \epsilon_{B}=0.18 \pm 0.09, A_{*}=2.3_{-0.7}^{+1.2}$, $E_{\mathrm{K}, \text { iso }}=\left(1.2_{-0.2}^{+0.3}\right) \times 10^{53} \mathrm{erg}, t_{\text {jet }}=(9.2 \pm 0.6) \times 10^{-2} \mathrm{~d}$, and $A_{\mathrm{V}}=0.13 \pm 0.01$, corresponding to a jet opening angle of $\theta_{\text {jet }}=3: 0 \pm 0.3$ and a beaming corrected kinetic energy of $E_{\mathrm{K}}=\left(1.6_{-0.3}^{0.5}\right) \times 10^{50} \mathrm{erg}$, the model match the data after the optical peak well (Figure 36). We present the correlation contours between the physical parameters in Figure 37 and the marginalized distributions for individual parameters in Figure 38.

The light curves before $0.04 \mathrm{~d}$ in Figure 36 are based on the same energy injection model as presented in Section 6.3.3. In this model, the energy increases by a factor of $\approx 27$ like in the the ISM case. However, the injection rate is not compatible with the maximum rate expected for a wind-like environment, similar to the other wind models (Appendices B, C, and D). We note that the optical and $\mathrm{X}$-ray frequencies are located above both the cooling frequency and $\nu_{\mathrm{m}}$, with $\nu_{\mathrm{c}}<\nu_{\mathrm{m}}<\nu_{\mathrm{opt}}<\nu_{\mathrm{X}}$ in this case, and in this regime, the light curves are independent of the circumburst density profile. Thus the measurements do not allow us to distinguish between a wind or ISM-like environment in the case of GRB 120404A. Clear detections of a wind-like circumburst environment in conjunction with a steep energy injection rate in the future will enable us to furthur probe the massive ejecta model of energy injection in GRB afterglows, and thereby to further constrain the mechanism responsible for plateaus and re-brightening events in GRB afterglows.

\section{REFERENCES}

Amati, L., Guidorzi, C., Frontera, F., et al. 2008, MNRAS, 391, 577 Andreev, M., Sergeev, A., \& Pozanenko, A. 2010a, GCN, 11166, 1 Andreev, M., Sergeev, A., \& Pozanenko, A. 2010b, GCN, 11168, 1 Andreev, M., Sergeev, A., \& Pozanenko, A. 2010c, GCN, 11191, 1 Andreev, M., Sergeev, A., \& Pozanenko, A. 2010d, GCN, 11201, 1 Andreev, M., Sergeev, A., Pozanenko, A., et al. 2010e, GCN, 11200, 1 Antonelli, L. A., Maund, J. R., Palazzi, E., et al. 2010, GCN, 10620, 1 Barthelmy, S. D., Barbier, L. M., Cummings, J. R., et al. 2005, SSRv, 120, 143 Barthelmy, S. D., Sakamoto, T., Markwardt, C. B., et al. 2012, GCN, 13120, 1 Berger, E., Price, P. A., Cenko, S. B., et al. 2005, Natur, 438, 988

Berger, E., Sari, R., Frail, D. A., et al. 2000, ApJ, 545, 56

Bernardini, M. G., Margutti, R., Chincarini, G., Guidorzi, C., \& Mao, J. 2011, A\&A, 526, A27

Blandford, R. D., \& McKee, C. F. 1977, MNRAS, 180, 343

Breeveld, A. A., \& Stratta, G. 2012, GCN, 13226, 1

Burrows, D. N., Hill, J. E., Nousek, J. A., et al. 2005b, SSRv, 120, 165

Burrows, D. N., Romano, P., Falcone, A., et al. 2005a, Sci, 309, 1833

Cenko, S. B., Fox, D. B., moon, D.-S., et al. 2006, PASP, 118, 1396

Cenko, S. B., Frail, D. A., Harrison, F. A., et al. 2010, ApJ, 711, 641

Cenko, S. B., Frail, D. A., Harrison, F. A., et al. 2011, ApJ, 732, 29

Chandra, P., Cenko, S. B., Frail, D. A., et al. 2008, ApJ, 683, 924

Chandra, P., \& Frail, D. A. 2010a, GCN, 11257, 1

Chandra, P., \& Frail, D. A. 2010b, GCN, 10650, 1

Chevalier, R. A., \& Li, Z.-Y. 2000, ApJ, 536, 195

Chiang, J., \& Dermer, C. D. 1999, ApJ, 512, 699

Chincarini, G., Mao, J., Margutti, R., et al. 2010, MNRAS, 406, 2113

Chincarini, G., Moretti, A., Romano, P., et al. 2007, ApJ, 671, 1903

Chornock, R., Berger, E., Fox, D., et al. 2010, GCN, 11164, 1

Collazzi, A. C. 2012, GCN, 13145, 1

Covino, S., D'Avanzo, P., Klotz, A., et al. 2008, MNRAS, 388, 347

Cucchiara, A., \& Tanvir, N. R. 2012, GCN, 13217, 1

Dai, Z. G., \& Lu, T. 1998, A\&A, 333, L87

Dall'Osso, S., Stratta, G., Guetta, D., et al. 2011, A\&A, 526, A121

de Cia, A., Vreeswijk, P. M., \& Jakobsson, P. 2010, GCN, 11170, 1

de Ugarte Postigo, A., Fatkhullin, T. A., Jóhannesson, G., et al. 2007, A\&A, 462, L57

de Ugarte Postigo, A., Lundgren, A., Martín, S., et al. 2012, A\&A, 538, A44 Dermer, C. D., Böttcher, M., \& Chiang, J. 2000a, ApJ, 537, 255

Dermer, C. D., Chiang, J., \& Mitman, K. E. 2000b, ApJ, 537, 785
Duffell, P. C., \& MacFadyen, A. I. 2014, arXiv:1407.8250

Eichler, D., \& Granot, J. 2006, ApJL, 641, L5

Elenin, L., Molotov, I., \& Pozanenko, A. 2010a, GCN, 11184, 1

Elenin, L., Molotov, I., \& Pozanenko, A. 2010b, GCN, 11234, 1

Evans, P. A., Beardmore, A. P., Page, K. L., et al. 2007, A\&A, 469, 379

Evans, P. A., Beardmore, A. P., Page, K. L., et al. 2009, MNRAS, 397, 1177

Falcone, A. D., Burrows, D. N., Lazzati, D., et al. 2006, ApJ, 641, 1010

Fan, Y., \& Piran, T. 2006, MNRAS, 369, 197

Fan, Y. Z., \& Wei, D. M. 2005, MNRAS, 364, L42

Filgas, R., Klose, S., \& Greiner, J. 2010, GCN, 10617, 1

Foreman-Mackey, D., Hogg, D. W., Lang, D., \& Goodman, J. 2013, PASP, 125,306

Frail, D. A., Waxman, E., \& Kulkarni, S. R. 2000, ApJ, 537, 191

Frail, D. A., Yost, S. A., Berger, E., et al. 2003, ApJ, 590, 992

Friedman, A. S., \& Bloom, J. S. 2005, ApJ, 627, 1

Gehrels, N., Chincarini, G., Giommi, P., et al. 2004, ApJ, 611, 1005

Geng, J. J., Wu, X. F., Li, L., Huang, Y. F., \& Dai, Z. G. 2014, ApJ, 792, 31 Ghirlanda, G., Nava, L., Ghisellini, G., \& Firmani, C. 2007, A\&A, 466, 127

Gorbovskoy, E., Lipunov, V., Kornilov, V., et al. 2010, GCN, 11178, 1

Gorbovskoy, E., Lipunov, V., Kornilov, V., et al. 2012, GCN, 13230, 1

Gorbovskoy, E. S., Lipunova, G. V., Lipunov, V. M., et al. 2012, MNRAS, 421,1874

Granot, J., Königl, A., \& Piran, T. 2006, MNRAS, 370, 1946

Granot, J., \& Kumar, P. 2006, MNRAS, 366, L13

Granot, J., Miller, M., Piran, T., Suen, W., \& Hughes, P. 2001, in Gamma-Ray Bursts in the Afterglow Era, ed. E. Costa, F. Frontera, \& J. Hjorth (Berlin, Heidelberg: Springer), 312 http://arxiv.org/pdf/astro-ph/0103038.pdf

Granot, J., Panaitescu, A., Kumar, P., \& Woosley, S. E. 2002, ApJL, 570, L61 Granot, J., \& Sari, R. 2002, ApJ, 568, 820

Greisen, E. W. 2003, Information Handling in Astronomy-Historical Vistas, Vol. 285

Guidorzi, C., Dichiara, S., Frontera, F., et al. 2015, ApJ, 801, 57

Guidorzi, C., Melandri, A., \& Japelj, J. 2012, GCN, 13209, 1

Guidorzi, C., Mundell, C. G., Harrison, R., et al. 2014, MNRAS, 438, 752

Guidorzi, C., Vergani, S. D., Sazonov, S., et al. 2007, A\&A, 474, 793

Harrison, F. A., Yost, S. A., Sari, R., et al. 2001, ApJ, 559, 123

Hascoet, R., Beloborodov, A. M., Daigne, F., \& Mochkovitch, R. 2015, arXiv: 1503.08333

Hascoët, R., Daigne, F., \& Mochkovitch, R. 2012, A\&A, 541, A88

Hentunen, V.-P., Nissinen, M., \& Salmi, T. 2010, GCN, 11173, 1

Hentunen, V.-P., Nissinen, M., \& Salmi, T. 2012, GCN, 13119, 1

Ho, P. T. P., Moran, J. M., \& Lo, K. Y. 2004, ApJL, 616, L1

Hou, S. J., Geng, J. J., Wang, K., et al. 2014, ApJ, 785, 113

Huang, Y. F., Dai, Z. G., \& Lu, T. 2002, MNRAS, 332, 735

Im, M., Choi, C., Jun, H., et al. 2010, GCN, 11208, 1

Immler, S., Barthelmy, S. D., Baumgartner, W. H., et al. 2010, GCN, 11159, 1 Jang, M., Im, M., \& Urata, Y. 2012, GCN, 13139, 1

Jester, S., Schneider, D. P., Richards, G. T., et al. 2005, AJ, 130, 873

Jin, Z. P., Yan, T., Fan, Y. Z., \& Wei, D. M. 2007, ApJL, 656, L57

Kalberla, P. M. W., Burton, W. B., Hartmann, D., et al. 2005, A\&A, 440, 775

Kann, D. A., Laux, U., \& Stecklum, B. 2010a, GCN, 11236, 1

Kann, D. A., Ludwig, F., \& Stecklum, B. 2010b, GCN, 11246, 1

Klotz, A., Gendre, B., Boer, M., \& Atteia, J. L. 2012, GCN, 13108, 1

Kobayashi, S. 2000, ApJ, 545, 807

Kobayashi, S., \& Zhang, B. 2003, ApJ, 597, 455

Kobayashi, S., \& Sari, R. 2000, ApJ, 542, 819

Kong, S. W., Wong, A. Y. L., Huang, Y. F., \& Cheng, K. S. 2010, MNRAS, 402, 409

Kopac, D., Dintinjana, B., \& Gomboc, A. 2010, GCN, 11177, 1

Kuin, N. P. M., Holland, S., \& Siegel, M. H. 2012, GCN, 13114, 1

Kumar, P., \& Piran, T. 2000, ApJ, 532, 286

Kuroda, D., Hanayama, H., Miyaji, T., et al. 2010a, GCN, 11258, 1

Kuroda, D., Hanayama, H., Miyaji, T., et al. 2010b, GCN, 11205, 1

Kuroda, D., Yanagisawa, K., Shimizu, Y., et al. 2010c, GCN, 11172, 1

Kuroda, D., Yanagisawa, K., Shimizu, Y., et al. 2010d, GCN, 11189, 1

Lacluyze, A., Haislip, J., Ivarsen, K., et al. 2012, GCN, 13109, 1

Landolt, A. U. 2009, AJ, 137, 4186

Laskar, T., Berger, E., Tanvir, N., et al. 2014, ApJ, 781, 1

Laskar, T., Berger, E., Zauderer, B. A., et al. 2013, ApJ, 776, 119

Lazzati, D., \& Perna, R. 2007, MNRAS, 375, L46

Lazzati, D., Rossi, E., Covino, S., Ghisellini, G., \& Malesani, D. 2002, A\&A, 396, L5

Li, L., Liang, E.-W., Tang, Q.-W., et al. 2012, ApJ, 758, 27

Liang, E. W., Zhang, B., O'Brien, P. T., et al. 2006, ApJ, 646, 351

Liang, E.-W., Zhang, B.-B., \& Zhang, B. 2007, ApJ, 670, 565

Lipkin, Y. M., Ofek, E. O., Gal-Yam, A., et al. 2004, ApJ, 606, 381 
Liu, X. W., Wu, X. F., \& Lu, T. 2008, A\&A, 487, 503

Malesani, D., Covino, S., D’Avanzo, P., et al. 2007, A\&A, 473, 77

Malesani, D., \& Palazzi, E. 2010, GCN, 10631, 1

Mangano, V., Holland, S. T., Malesani, D., et al. 2007, A\&A, 470, 105

Margutti, R., Bernardini, G., Barniol Duran, R., et al. 2011a, MNRAS, 410, 1064

Margutti, R., Chincarini, G., Granot, J., et al. 2011b, MNRAS, 417, 2144

Margutti, R., Genet, F., Granot, J., et al. 2010a, MNRAS, 402, 46

Margutti, R., Guidorzi, C., Chincarini, G., et al. 2010b, MNRAS, 406, 2149

Margutti, R., Zaninoni, E., Bernardini, M. G., et al. 2013, MNRAS, 428, 729

Marshall, F. E., Antonelli, L. A., Burrows, D. N., et al. 2011, ApJ, 727, 132

Marshall, F. E., Beardmore, A. P., Gelbord, J. M., et al. 2010, GCN, 10612, 1

Melandri, A., Virgili, F. J., Guidorzi, C., et al. 2014, A\&A, 572, A55

Moin, A., Chandra, P., Miller-Jones, J. C. A., et al. 2013, ApJ, 779, 105

Moin, A., Tingay, S., Phillips, C., et al. 2010, GCN, 10832, 1

Monfardini, A., Kobayashi, S., Guidorzi, C., et al. 2006, ApJ, 648, 1125

Moody, J. W., Laney, D., Pearson, R., \& Pace, C. 2010, GCN, 10665, 1

Morgan, A. N. 2012, GCN, 13143, 1

Nakar, E., \& Granot, J. 2007, MNRAS, 380, 1744

Nousek, J. A., Kouveliotou, C., Grupe, D., et al. 2006, ApJ, 642, 389

O'Brien, P. T., Willingale, R., Osborne, J., et al. 2006, ApJ, 647, 1213

Pagani, C., Morris, D. C., Kobayashi, S., et al. 2006, ApJ, 645, 1315

Page, K. L., \& Immler, S. 2010, GCN, 11171, 1

Panaitescu, A., \& Kumar, P. 2000, ApJ, 543, 66

Panaitescu, A., \& Kumar, P. 2001, ApJ, 554, 667

Panaitescu, A., \& Kumar, P. 2002, ApJ, 571, 779

Panaitescu, A., \& Mészáros, P. 1998, ApJ, 501, 772

Panaitescu, A., Mészáros, P., Gehrels, N., Burrows, D., \& Nousek, J. 2006, MNRAS, 366, 1357

Panaitescu, A., Vestrand, W. T., \& Woźniak, P. 2013, MNRAS, 433, 759

Pandey, S. B., \& Zheng, W. 2010, GCN, 11179, 1

Pei, Y. C. 1992, ApJ, 395, 130

Perley, D. A., Alatalo, K., \& Horesh, A. 2012, GCN, 13175, 1

Perna, R., Armitage, P. J., \& Zhang, B. 2006, ApJL, 636, L29

Phinney, E. S. 1989, in IAU Symp. 136, The Center of the Galaxy, ed.

M. Morris (Cambridge: Cambridge Univ. Press), 543

Piro, L., Amati, L., Antonelli, L. A., et al. 1998, A\&A, 331, L41

Pritchard, T. A., \& Immler, S. 2010, GCN, 11176, 1

Proga, D., \& Zhang, B. 2006, MNRAS, 370, L61

Rees, M. J., \& Meszaros, P. 1998, ApJL, 496, L1

Rhoads, J. E. 1999, ApJ, 525, 737

Romano, P., Moretti, A., Banat, P. L., et al. 2006, A\&A, 450, 59

Roming, P. W. A., Kennedy, T. E., Mason, K. O., et al. 2005, SSRv, 120, 95

Rujopakarn, W., \& Flewelling, H. 2012, GCN, 13106, 1

Rumyantsev, V., Shakhovkoy, D., \& Pozanenko, A. 2010, GCN, 11255, 1

Sahu, D. K., Anupama, G. C., \& Pandey, S. B. 2012, GCN, 13185, 1

Sahu, D. K., Arora, S., Singh, N. S., \& Kartha, S. S. 2010a, GCN, 11197, 1

Sahu, D. K., Bhatt, B. C., \& Arora, S. 2010b, GCN, 11175, 1

Salvaterra, R., Della Valle, M., Campana, S., et al. 2009, Natur, 461, 1258

Sari, R., \& Esin, A. A. 2001, ApJ, 548, 787

Sari, R., \& Mészáros, P. 2000, ApJL, 535, L33

Sari, R., Narayan, R., \& Piran, T. 1996, ApJ, 473, 204

Sari, R., Piran, T., \& Halpern, J. P. 1999, ApJL, 519, L17
Sari, R., Piran, T., \& Narayan, R. 1998, ApJL, 497, L17

Sault, R. J., Teuben, P. J., \& Wright, M. C. H. 1995, in ASP Conf. Ser. 77, Astronomical Data Analysis Software and Systems IV, ed. R. A. Shaw, H. E. Payne, \& J. J. E. Hayes (San Francisco, CA: ASP), 433

Schaefer, B. E., Gerardy, C. L., Höflich, P., et al. 2003, ApJ, 588, 387

Shao, L., \& Dai, Z. G. 2007, ApJ, 660, 1319

Shen, R.-F., Barniol Duran, R., \& Kumar, P. 2008, MNRAS, 384, 1129

Siegel, M. H., Barthelmy, S. D., Burrows, D. N., et al. 2012, GCN, 13105, 1

Siegel, M. H., \& Marshall, F. 2010, GCN, 10625, 1

Sposetti, S., \& Immler, S. 2010, GCN, 11213, 1

Stratta, G., Barthelmy, S. D., Baumgartner, W. H., et al. 2012, GCN, 13208, 1

Sudilovsky, V., Nicuesa Guelbenzu, A., \& Greiner, J. 2012, GCN, 13129, 1

Tagliaferri, G., Goad, M., Chincarini, G., et al. 2005, Natur, 436, 985

Tello, J. C., Sanchez-Ramirez, R., Gorosabel, J., et al. 2012, GCN, 13118, 1

Toma, K., Ioka, K., Yamazaki, R., \& Nakamura, T. 2006, ApJL, 640, L139

Totani, T. 1998, ApJL, 502, L13

Uhm, Z. L., \& Zhang, B. 2014, ApJ, 789, 39

Uhm, Z. L., Zhang, B., Hascoët, R., et al. 2012, ApJ, 761, 147

Ukwatta, T. N., Barthelmy, S. D., Baumgartner, W. H., et al. 2012, GCN, 13220,1

Ukwatta, T. N., Sakamoto, T., Gehrels, N., \& Dhuga, K. S. 2010, GCN, 11198, 1 Updike, A. C., Hartmann, D. H., \& de Pree, C. 2010a, GCN, 10637, 1

Updike, A. C., Hartmann, D. H., Keel, W., \& Darnell, E. 2010b, GCN, 11174, 1

Updike, A. C., Hartmann, D. H., \& Murphy, B. 2010c, GCN, 10619, 1

Urata, Y., Huang, K., Takahashi, S., et al. 2014, ApJ, 789, 146

van der Horst, A. J., Kamble, A. P., Wijers, R. A. M. J., et al. 2010a, GCN, 10647, 1

van der Horst, A. J., Wiersema, K., Kamble, A. P., et al. 2010b, GCN, 11221, 1

Volnova, A., Pozanenko, A., Ibrahimov, M., Hafizov, B., \& Satovski, B. 2010, GCN, 11266, 1

Volnova, A., Sinyakov, E., Varda, D., \& Molotov, I. 2012, GCN, 13198, 1

Wang, X.-Y., Li, Z., \& Mészáros, P. 2006, ApJL, 641, L89

Wei, D. M., \& Lu, T. 1998, ApJ, 505, 252

Willingale, R., Genet, F., Granot, J., \& O'Brien, P. T. 2010, MNRAS, 403, 1296

Wilson, J. C., Eikenberry, S. S., Henderson, C. P., et al. 2003, Proc. SPIE, 4841, 451

Xin, L. P., Pozanenko, A., Kann, D. A., et al. 2012, MNRAS, 422, 2044

Xin, L. P., Wei, J. Y., Qiu, Y. L., et al. 2012a, GCN, 13150, 1

Xin, L. P., Wei, J. Y., Qiu, Y. L., et al. 2012b, GCN, 13221, 1

Yoshida, M., Sasada, M., Komatsu, T., \& Kawabata, K. S. 2010, GCN 11190,1

Yost, S. A., Frail, D. A., Harrison, F. A., et al. 2002, ApJ, 577, 155

Yost, S. A., Harrison, F. A., Sari, R., \& Frail, D. A. 2003, ApJ, 597, 459

Yu, Y. B., Wu, X. F., Huang, Y. F., et al. 2015, MNRAS, 446, 3642

Yu, Y. W., \& Dai, Z. G. 2007, A\&A, 470, 119

Zauderer, A., Laskar, T., \& Berger, E. 2012, GCN, 13231, 1

Zhang, B., Fan, Y. Z., Dyks, J., et al. 2006, ApJ, 642, 354

Zhang, B., Liang, E., Page, K. L., et al. 2007, ApJ, 655, 989

Zhang, B., \& Mészáros, P. 2001, ApJL, 552, L35

Zhang, B., \& Mészáros, P. 2002, ApJ, 566, 712

Zhao, X.-H., Mao, J., Xu, D., \& Bai, J.-M. 2012, GCN, 13122, 1

Zou, Y. C., Wu, X. F., \& Dai, Z. G. 2005, MNRAS, 363, 93 